\title{
Modeling of Wind Phenomena and Analysis of Their Effects on UAV Trajectory Tracking Performance
}

\author{
Jessica Cristine Da Costa Siqueira
}

Follow this and additional works at: https://researchrepository.wvu.edu/etd

\section{Recommended Citation}

Siqueira, Jessica Cristine Da Costa, "Modeling of Wind Phenomena and Analysis of Their Effects on UAV Trajectory Tracking Performance" (2017). Graduate Theses, Dissertations, and Problem Reports. 7347. https://researchrepository.wvu.edu/etd/7347

This Thesis is protected by copyright and/or related rights. It has been brought to you by the The Research Repository @ WVU with permission from the rights-holder(s). You are free to use this Thesis in any way that is permitted by the copyright and related rights legislation that applies to your use. For other uses you must obtain permission from the rights-holder(s) directly, unless additional rights are indicated by a Creative Commons license in the record and/ or on the work itself. This Thesis has been accepted for inclusion in WVU Graduate Theses, Dissertations, and Problem Reports collection by an authorized administrator of The Research Repository @ WVU. For more information, please contact researchrepository@mail.wvu.edu. 


\title{
Modeling of Wind Phenomena and Analysis of Their Effects on UAV Trajectory Tracking Performance
}

\author{
Jessica Cristine Da Costa Siqueira
}

\author{
Thesis submitted to the \\ Benjamin M. Statler College of Engineering and Mineral Resources \\ at West Virginia University \\ in partial fulfillment of the requirements for the degree of

\section{Master of Science} \\ in \\ Aerospace Engineering
}

Mario G. Perhinschi, Ph.D., Chair

Patrick Browning, Ph.D.

Christopher Griffin, Ph.D.

Department of Mechanical and Aerospace Engineering

Morgantown, West Virginia

May 2017

Keywords: Wind Modeling, UAVs, Trajectory Tracking Control, Wind Shear, Wind Gust

Copyright $^{\odot} 2017$ Jessica Siqueira 
ProQuest Number: 10275311

All rights reserved

INFORMATION TO ALL USERS

The quality of this reproduction is dependent upon the quality of the copy submitted.

In the unlikely event that the author did not send a complete manuscript and there are missing pages, these will be noted. Also, if material had to be removed, a note will indicate the deletion.

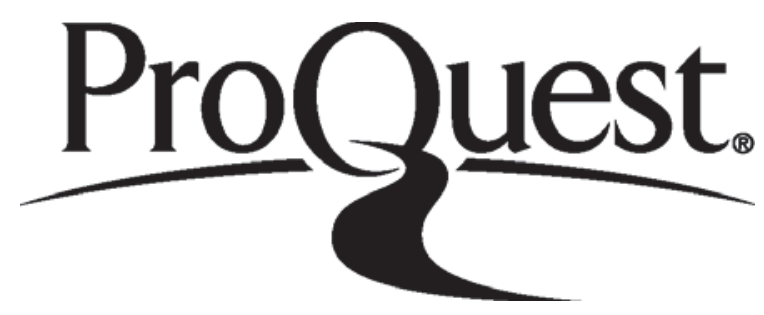

ProQuest 10275311

Published by ProQuest LLC (2017). Copyright of the Dissertation is held by the Author.

All rights reserved.

This work is protected against unauthorized copying under Title 17, United States Code Microform Edition (C) ProQuest LLC.

ProQuest LLC.

789 East Eisenhower Parkway

P.O. Box 1346

Ann Arbor, Ml $48106-1346$ 


\begin{abstract}
Modeling of Wind Phenomena and Analysis of Their Effects on UAV Trajectory Tracking Performance
\end{abstract}

\title{
Jessica Cristine Da Costa Siqueira
}

The use of autonomous unmanned aerial vehicles (UAV) has increased greatly over the years and is predicted to increase even more in the future. Thus, it is essential that these vehicles are able to fly safely with adequate performance under normal and abnormal conditions. In this research effort, the objective was to create a wind model to analyze the effects of atmospheric phenomena on trajectory tracking control of UAVs. A simplified model was developed and implemented within the WVU UAV simulation environment in order to simulate atmospheric phenomena, such as, constant wind with turbulence, wind gust and wind shear. Graphical user interfaces allow the setup of diverse simulation scenarios including constant wind and gusts in any direction and of any magnitude, different levels of turbulence and spatial variation of wind vector components in any direction (wind shear). The factors of the experimental grid also include, fixed parameter and adaptive trajectory tracking control laws, different 2-dimensional and 3-dimensional commanded trajectories and aircraft actuator failures.

Analysis of trajectory tracking performance relied on using composite indices based on trajectory tracking errors and control activity. Results show that, as the magnitude of the wind phenomena increases, the trajectory tracking degrades significantly for both adaptive and fixed parameter control laws, up to the point of loss of control. Control activity exhibits much less sensitivity. While adaptive control laws generally perform better, they present a greater degradation relative to nominal conditions than their fixed parameter counterpart. These results lead to the observation that specific adaptive mechanisms successful in handling a variety of other abnormal flight conditions may be less effective under wind. The direction of the wind relative to the aircraft proved important. In particular, downward wind components degrade significantly trajectory tracking and can easily lead to loss of control especially in combination with severe turbulence. The combination of actuator failures and wind conditions demonstrated that the adaptive controller presents higher performance than the fixed parameter controller.

This study reveals that UAV flight under wind phenomena may pose specific challenges in terms of trajectory tracking control laws design. Due to their typically reduced size, UAVs possess increased sensitivity to wind phenomena, which must be specifically addressed to improve safety and performance. 


\section{DEDICATION}

To my family and friends who always believed in me and helped me through tough times. When I could not walk, you all carried me. I could not have done it without you, thank you. 


\section{ACKNOWLEDGEMENTS}

I would like to thank Dr. Perhinschi and Dr. Mucino for their great advice, patience and guidance throughout my education. I had a great learning experience at West Virginia University and my dream of pursuing a master degree became possible thanks to you.

To my friends Bruno, Paolo, Mario and Al who helped me as study buddies and Ghassan for having my back, giving me advice and helping throughout this research. Thank you so much, I absolutely could not have done it without you.

Special thanks to my dear friends Christy and family, Matt, Jennifer, Priyash, Mofe and Jeff for your help and support during times of need, what you did for me was far beyond of what I could have ever hoped for, thank you so much.

To my mom and sister, you are the most important people in my life, I love you very much, I hope that I can reciprocate what you did for me and someday compensate the time I spent away from you.

Finally, I would like to thank my dad for always believing in me and to my whole family for always going out of their way to make my wildest dreams come true. I only got this far because of your support, thank you. 


\section{TABLE OF CONTENTS}

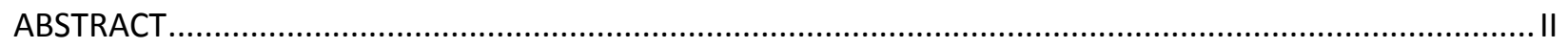

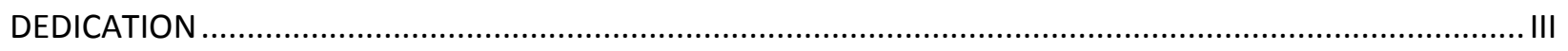

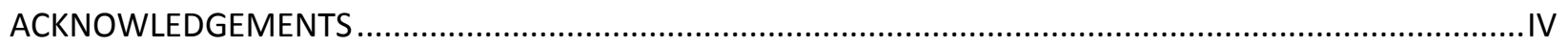

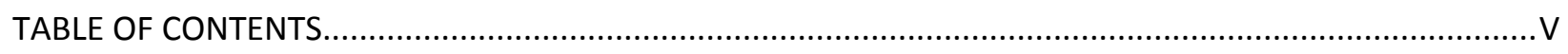

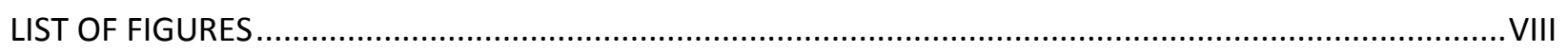

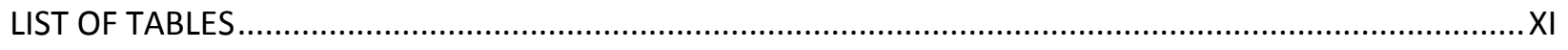

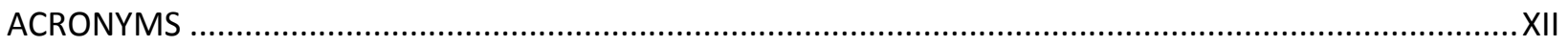

NOMENCLATURE

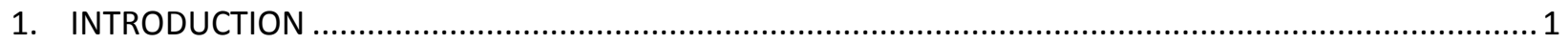

1.1. Background and Motivation ................................................................................................. 1

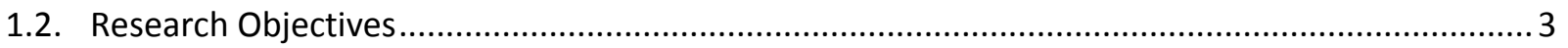

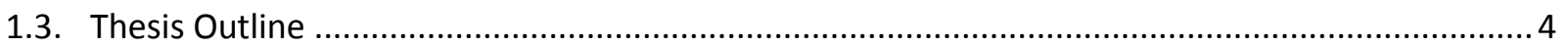

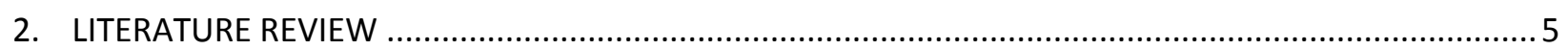

2.1. Aircraft Accidents Related To Wind and Failures ..................................................................... 5

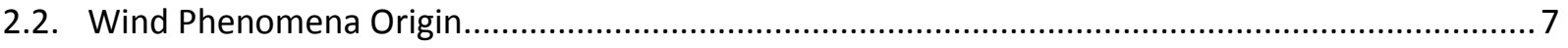

2.3. Aircraft Abnormal Conditions ................................................................................................... 15

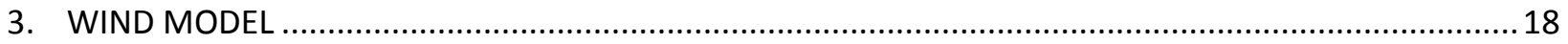

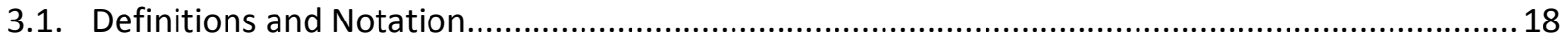

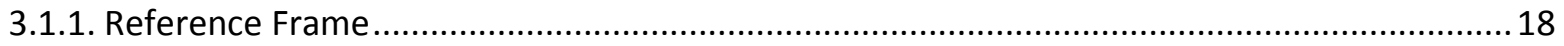

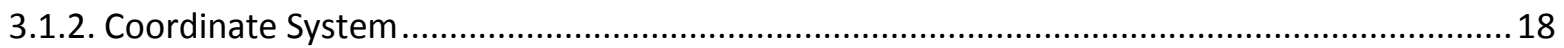

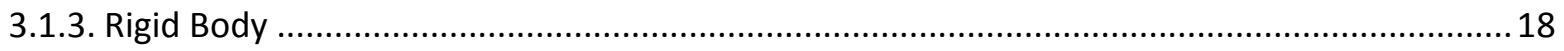

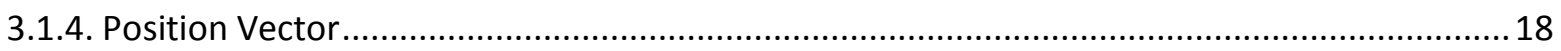

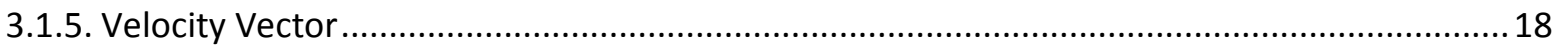

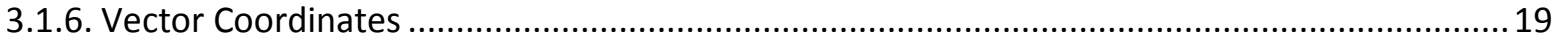

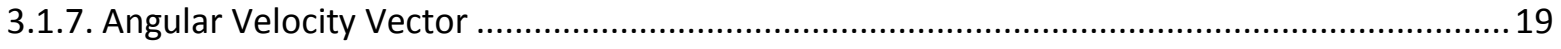

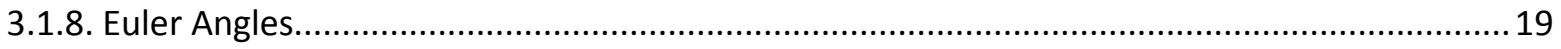

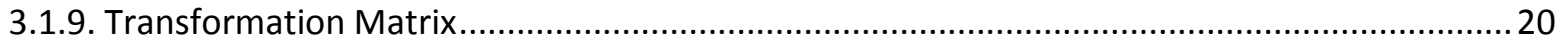

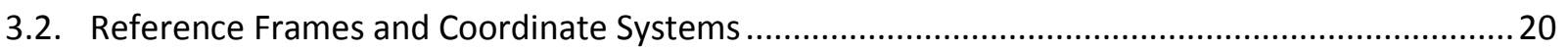

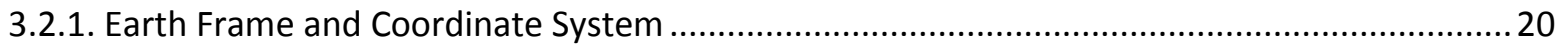

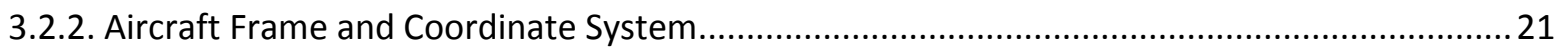

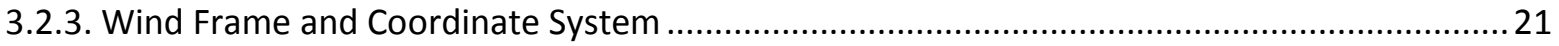




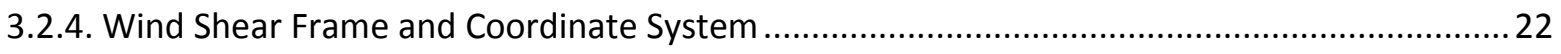

3.2.5. Gust Frame and Coordinate System ................................................................................... 22

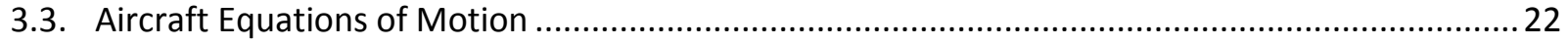

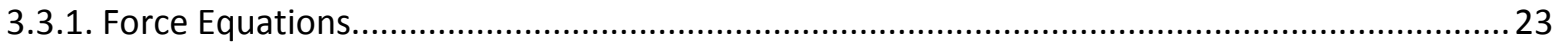

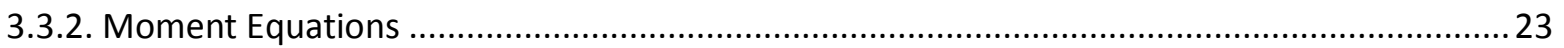

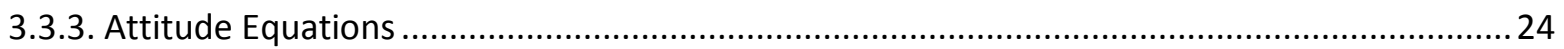

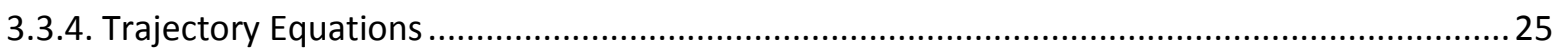

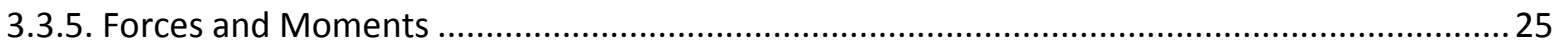

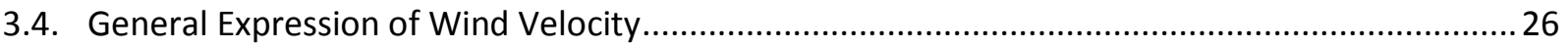

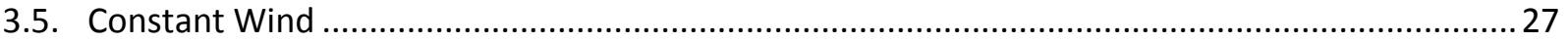

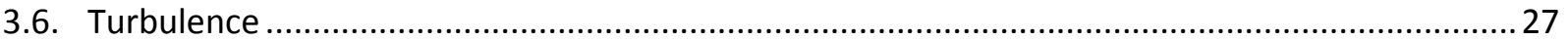

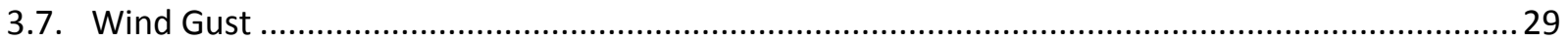

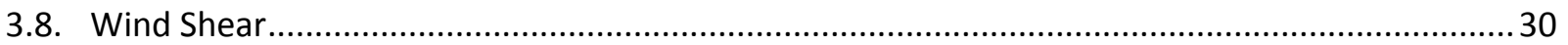

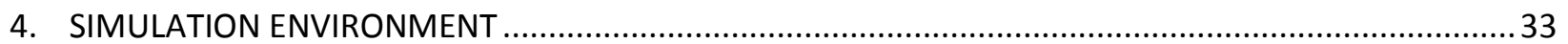

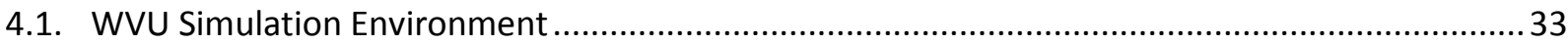

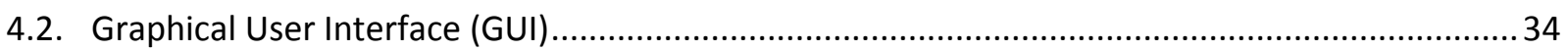

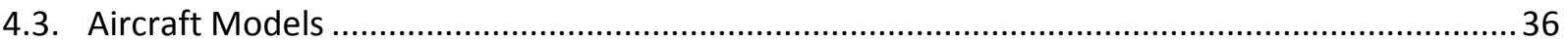

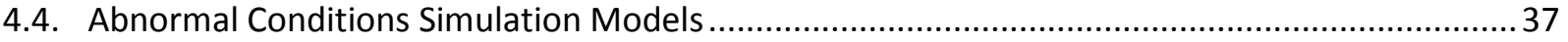

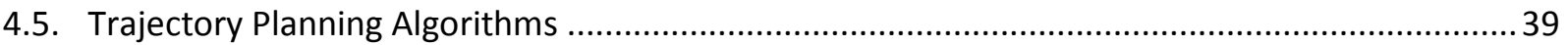

4.6. Trajectory Tracking Controllers............................................................................................ 42

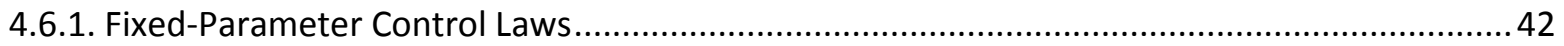

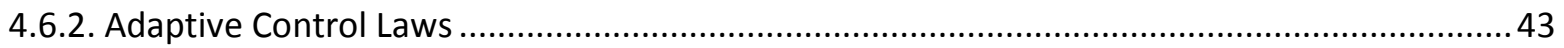

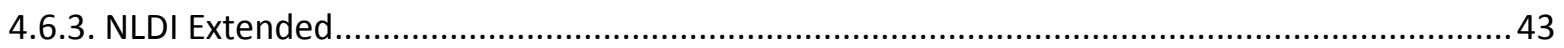

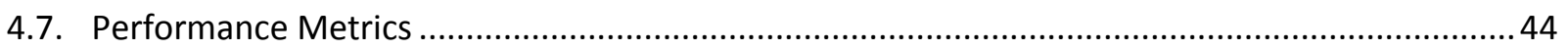

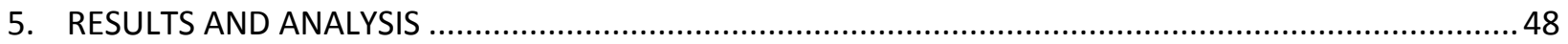

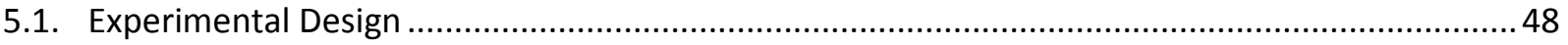

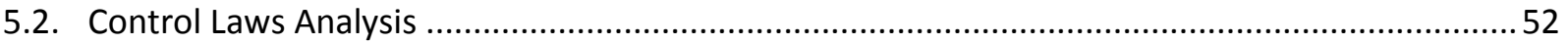

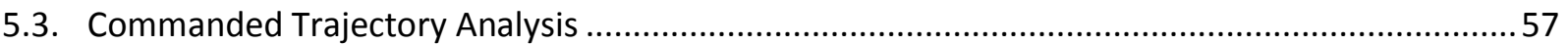

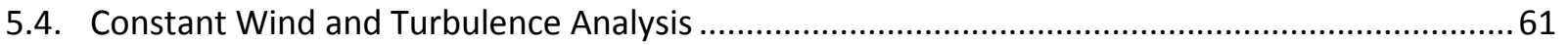

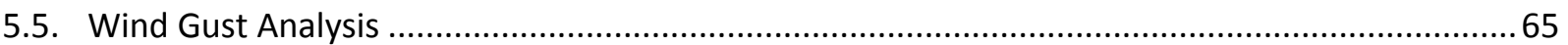

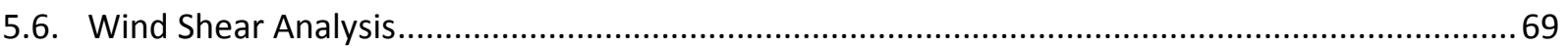

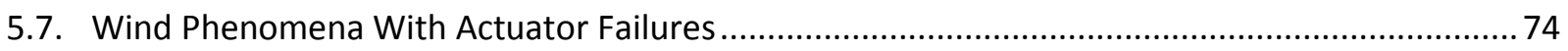

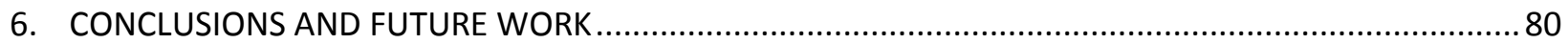




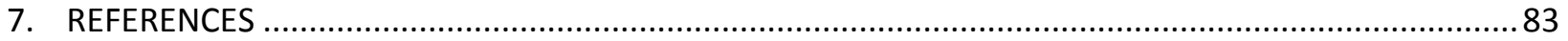

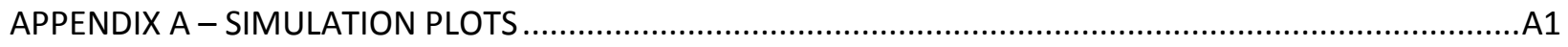




\section{LIST OF FIGURES}

Figure 1 - Coriolis Force Effect on Earth Atmosphere and Rotating Surfaces [12]................... 8

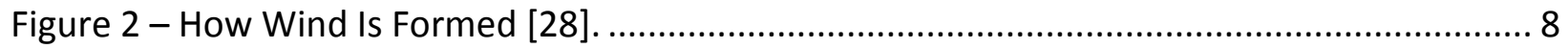

Figure 3- Relative Velocity and Wind Speed [29]............................................................... 9

Figure 4 - Wind Speed Vector Addition [29]. ..................................................................... 9

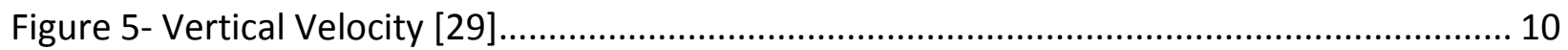

Figure 6 - Aircraft A380 Experiencing 60 mph Crosswind During Landing [32]...................... 10

Figure 7- Downdraft and Updraft Sketch [31] .................................................................. 11

Figure 8- Wind Gusts Effects on Aircraft Flight Path [35] ..................................................... 11

Figure 9- Wind Shear Encounter During Takeoff After Liftoff [42]........................................ 13

Figure 10- Wind Shear Effect During Landing [42] ............................................................. 13

Figure 11- Right Stabilator With Positive Deflection. ............................................................. 16

Figure 12- Right Aileron With Positive Deflection............................................................... 16

Figure 13- Right Rudder Positive Deflection................................................................... 17

Figure 14- Relative Position of the Body and Earth CS [58]............................................... 20

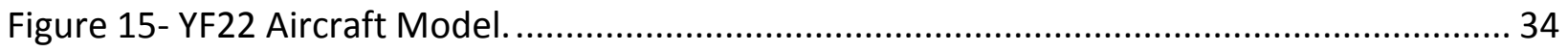

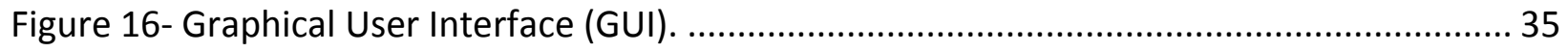

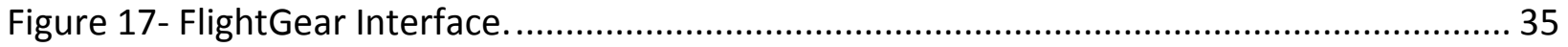

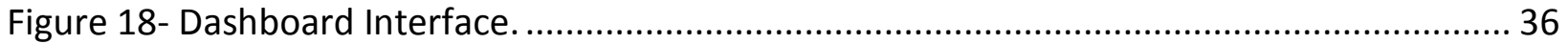

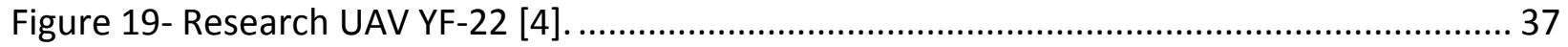

Figure 20- GUls for Constant Wind, Wind Gust and Wind Shear Models. ............................... 39

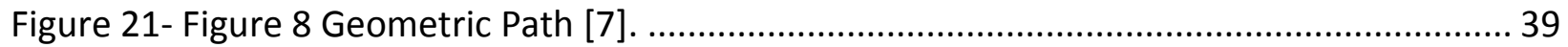

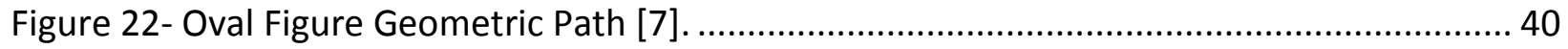

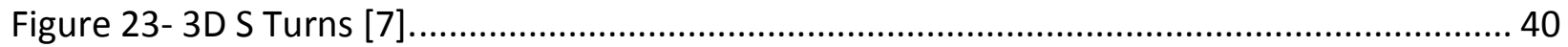

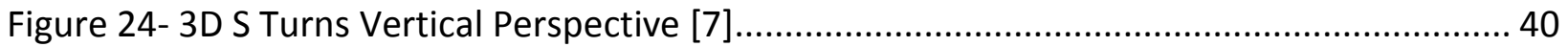

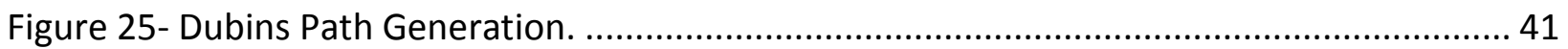

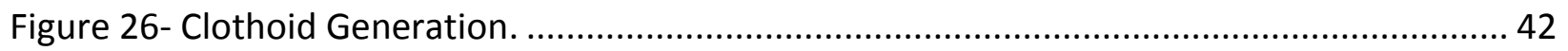

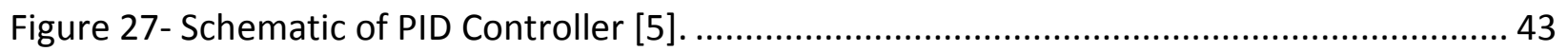

Figure 28- Schematic of NLDI Extended Controller. ......................................................... 44

Figure 29-Total Performance Index Contributions. ............................................................. 47

Figure 30- Tracking Index for Oval Trajectory Under Strong Constant Wind (Adaptive PPID). ... 53

Figure 31- Tracking Index for Oval Trajectory Under Strong Constant Wind (Fixed PPID). ......... 53 
Figure 32- Tracking Index for Oval Trajectory Under Strong Constant Wind for NLDI Extended (Fixed vs Adaptive). 54

Figure 33- Activity Index for Figure 8 Trajectory Under Strong Wind Shear (Adaptive PPID)...... 55

Figure 34- Activity Index for Figure 8 Trajectory Under Strong Wind Shear (Fixed PPID)........... 55

Figure 35- Activity Index for Oval Trajectory Under Strong Constant Wind (Fixed PPID)........... 56

Figure 36- Activity Index for Oval Trajectory Under Strong Constant Wind for NLDI Extended

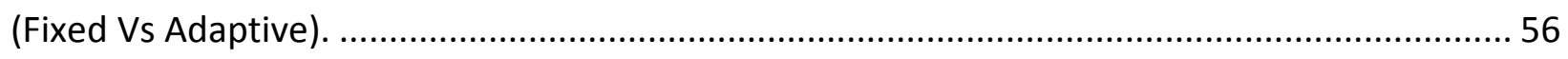

Figure 37- Tracking Index for Clothoid Trajectory Under Low Constant Wind (Adaptive PPID). . 57

Figure 38- Tracking Index for Dubins Trajectory Under Low Constant Wind (Adaptive PPID)..... 57

Figure 39- Tracking Index for 3D S Turns Trajectory Under Low Constant Wind (Fixed PPID). ... 58

Figure 40- Tracking Index for Dubins Trajectory Under Low Constant Wind (Fixed PPID). ......... 59

Figure 41- Trajectory Index for 3D S Turns Under Low Wind Gust (Adaptive PPID). .................. 59

Figure 42- Trajectory Index for 3D S Turns Under Low Wind Shear (Fixed PPID). .................... 60

Figure 43- Activity Index for Oval Trajectory Under Low Wind Gust (Fixed PPID) ..................... 60

Figure 44- Activity Index for 3D S Turns Trajectory Under Low Wind Gust (Fixed PPID)............ 61

Figure 45- Tracking Index for Figure 8 Trajectory Under Low Constant Wind (Fixed PPID)......... 62

Figure 46- Tracking Index for Figure 8 Trajectory Under Strong Constant Wind (Fixed PPID)..... 62

Figure 47- Tracking Index for Clothoid trajectory Under Strong Constant Wind (Fixed PPID)..... 63

Figure 48- Tracking Index for Dubins trajectory Under Strong Constant Wind (Fixed PPID)....... 63

Figure 49- Tracking Index for 3D S Turns Trajectory Under Low Constant Wind (Adaptive PPID).

Figure 50- Tracking Index for 3D S Trajectory Under Strong Constant Wind (Fixed PPID). ......... 65

Figure 51- Tracking Index for Figure 8 Trajectory Under Strong Wind Gust (Adaptive PPID)...... 66

Figure 52- Tracking Index for Figure 8 Trajectory Under Strong Wind Gust (Fixed PPID)........... 66

Figure 53- Tracking Index for Oval Trajectory Under Strong Wind Gust (Fixed PPID). ............... 67

Figure 54- Tracking Index for Oval Trajectory Under Strong Wind Gust (Adaptive PPID)........... 67

Figure 55- Tracking Index for 3D S Turns trajectory Under Strong Wind Gust (Adaptive PPID). . 68

Figure 56- Tracking Index for 3D S Turns trajectory Under Low Wind Gust (Fixed PPID)........... 68

Figure 57- Tracking Index for Figure 8 trajectory Under Low Wind Gust (Fixed PPID). .............. 69

Figure 58- Tracking Index for Figure 8 trajectory Under Low Wind Gust (Adaptive PPID). ......... 69

Figure 59- Tracking Index for Figure 8 Under Low Wind Shear (Fixed PPID). ........................... 70

Figure 60- Tracking Index for Figure 8 Under Strong Wind Shear (Fixed PPID). ....................... 71

Figure 61- Tracking Index for Oval Trajectory Under Strong Wind Shear (Fixed PPID)............... 71

Figure 62- Tracking Index for Oval Trajectory Under Strong Wind Shear (Adaptive PPID)......... 72 
Figure 63- Tracking Index for 3D S Turns trajectory Under Strong Wind Shear (Fixed PPID) ..... 72 Figure 64- Tracking Index for 3D S Turns trajectory Under Strong Wind Shear (Adaptive PPID). 73 Figure 65- Tracking Index for Oval Trajectory Under Low Wind Shear (Adaptive PPID)............. 73 Figure 66- Tracking Index for Oval Trajectory Under Low Wind Shear (Fixed PPID).................. 74

Figure 67- Tracking Index for Oval trajectory Under Wind Gust and Aileron Failure. ................ 75

Figure 68- Control Activity for Oval trajectory Under Wind Gust and Aileron Failure................ 75

Figure 69- Tracking Index for Under Constant Wind and Aileron Failure (Adaptive PPID)......... 76

Figure 70- Tracking Index for Under Constant Wind and Aileron Failure (Fixed PPID).............. 76

Figure 71- Control Activity for Under Constant Wind and Aileron Failure (Adaptive PPID). ....... 77

Figure 72- Control Activity for Under Constant Wind and Aileron Failure (Fixed PPID). ............ 77

Figure 73- Tracking Index for Oval Trajectory Under Wind Gust and Stabilator Failure............. 78

Figure 74- Control Activity for Oval Trajectory Under Wind Gust and Stabilator Failure........... 78

Figure 75- Tracking Index for Oval Trajectory Under Constant Wind and Stabilator Failure....... 79

Figure 76- Tracking Index for Oval Trajectory Under Constant Wind and Stabilator Failure...... 79 


\section{LIST OF TABLES}

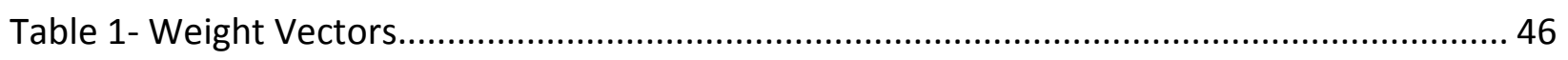

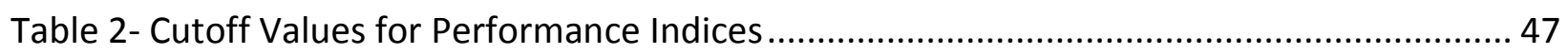

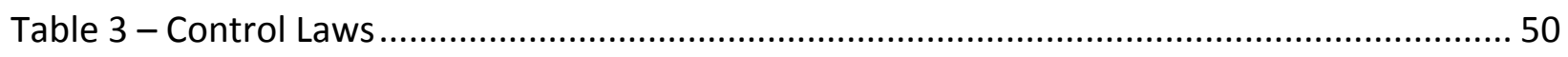

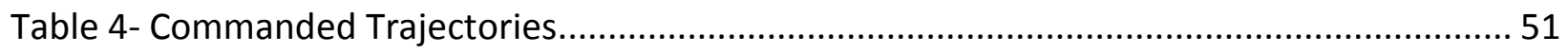

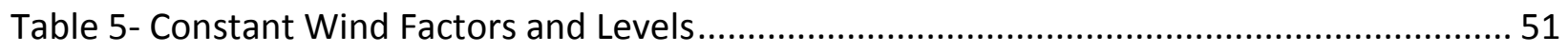

Table 6 - Wind Gust Factors and Levels ......................................................................... 51

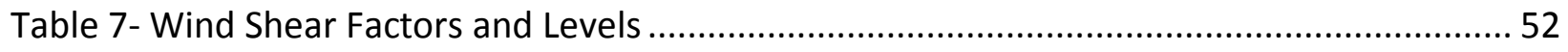

Table 8- Actuator Failures Factors and Levels .................................................................. 52 


\section{ACRONYMS}

\begin{tabular}{|c|c|}
\hline Symbol & Description \\
\hline CA & Control Activity \\
\hline CS & Coordinate System \\
\hline EKF & Extended Kalman Filter \\
\hline GPS & Global Positioning System \\
\hline GUI & Graphical User Interface \\
\hline LOC & Loss of Control \\
\hline NLDI & Nonlinear Dynamic Inversion \\
\hline PCA & Parametric Computational Aerodynamics \\
\hline $\mathrm{PI}$ & Performance Index \\
\hline PPID & Position Proportional Integral Derivative \\
\hline RF & Reference Frame \\
\hline STD & Standard Deviation \\
\hline $\mathrm{TT}$ & Trajectory Tracking \\
\hline UAS & Unmanned Aerial Systems \\
\hline UAV & Unmanned Aerial Vehicle \\
\hline WVU & West Virginia University \\
\hline
\end{tabular}




\section{NOMENCLATURE}

\begin{tabular}{|c|c|c|}
\hline Symbol & $\begin{array}{c}\text { Description } \\
\text { English }\end{array}$ & Units \\
\hline e & Error & - \\
\hline$\vec{V}$ & Vector & - \\
\hline$\vec{W}$ & Wind Vector & - \\
\hline $\mathrm{F}$ & Filter & - \\
\hline $\mathrm{R}$ & Region of Physical Space & - \\
\hline \multirow[t]{2}{*}{$\vec{r}$} & Position Vector & \\
\hline & Greek & \\
\hline$\phi$ & Roll Angle & rad \\
\hline$\Psi$ & Yaw Angle & rad \\
\hline$\theta$ & Pitch angle & rad \\
\hline$\delta$ & Control Surface Deflection & degree \\
\hline$\sigma$ & Standard Deviation & - \\
\hline$\Omega$ & Spatial Frequency & - \\
\hline$\Phi$ & Power Spectral Density & - \\
\hline$\Delta \Omega$ & Spatial Integration Step & - \\
\hline \multirow[t]{2}{*}{$\vec{\omega}$} & Angular Rate & $\mathrm{rad} / \mathrm{s}$ \\
\hline & Subscripts & \\
\hline $\mathrm{E}$ & Earth Axes & - \\
\hline B & Body Axes & - \\
\hline W & Constant Wind & - \\
\hline xy & Horizontal Plane & - \\
\hline$x y z$ & 3 Dimension Space & - \\
\hline
\end{tabular}




\begin{tabular}{ccc} 
G & Wind Gust & - \\
S & Wind Shear & - \\
\hline$a$ & Aileron & - \\
\hline e & Elevator & - \\
$\mathrm{r}$ & Rudder & - \\
$\mathrm{t}$ & Throttle & - \\
$\mathrm{f}$ & Filter & - \\
$\mathrm{n}$ & Noise & - \\
$\mathrm{t}$ & Time &
\end{tabular}




\section{INTRODUCTION}

\subsection{Background and Motivation}

An unmanned aerial vehicle (UAV) or "drone", is an aircraft capable of flying without any pilot on board; instead, the vehicle is remotely controlled by an operator on the ground [1] or is flying autonomously based on advanced on board control systems. It is expected that such vehicles perform a variety of tasks, while following the commanded trajectory in the most precise and safe manner. The vehicle, in conjunction with ground station, communications, payload and all other support components, is referred to as an unmanned aerial system (UAS). A wide range of different controllers were developed to meet this purpose, they can be robust or adaptive control laws types. Although many efforts in creating good controllers have been done, little has been published about the performance of the controller when the vehicle is under upset flight conditions or on how much those conditions can affect, even jeopardize, the success of the task given to the UAV [2].

The use of UAVs has increased greatly in recent years, especially in the military field for reconnaissance, transportation of supplies or combat [2 and 3]. Additionally, UAVs have gained popularity within civil and scientific use, such as traffic monitoring, surveillance, geological survey, visual and thermal imaging of a region and forest fire detection [4]. Consequently, the possibilities for their use are promising and endless due to their capability of flying autonomously or remotely controlled.

Whenever overflying an area is deemed too dangerous to risk a pilot's life or even if simply inconvenient or unnecessary to involve a human pilot, UAVs may prove to be extremely useful and reliable. UAVs are capable of successfully accomplishing many different, tedious, repetitive and long missions autonomously or with minimum human interaction [5]. Due to their on board technology, they are safe and less costly than manned aircraft. Significant reduction in costs associated to fuel, maintenance, and pilot operation, may be achieved by increasing the autonomy of the UAV.

In order to safely fly autonomously and perform diverse missions, UAVs utilizes control algorithms to ensure that the aircraft is correctly following the given trajectory. There are two major elements that are involved in automatic flight, they are the trajectory planning and trajectory tracking algorithms [4]. 
Trajectory planning algorithms have the purpose of creating a flyable path for the aircraft in between the starting and the final points of the mission such that specific missions can be accomplished. There can be a wide range of different possibilities of paths planned allowing for the creation of an obstacle or risk zone avoidance, to a point of interest observation [6], including a variety of optimization criteria. Nonetheless, the path planner must be capable of generating a flight path that the aircraft will attempt to follow using the controllers (trajectory tracking algorithms).

Although good controllers are possible to be implemented, one should keep in mind that it is extremely difficult to control an aircraft, due to its kinematics and nonlinear dynamics [4]. Models of aircraft are only approximations made based on data available, but contain uncertainties that need to be accounted for when developing good trajectory controllers.

In addition to model imprecisions resultant from approximations, other factors also account for inaccuracies within the model. An example of factors that can affect the model of the aircraft, are external forces or atmospheric disturbances associated with wind such as wind shear or gusts. While common controllers are effective and achieve good performance under nominal conditions, relying on these controllers might end up in poor or catastrophic results under upset flight conditions. Hence, there is another class of controllers created to meet this problem, they are called adaptive controllers [ 4 and 7 ].

The operational scheme of adaptive controllers is to vary or modify their parameters or structure in order to meet good levels of performance (the aircraft has only small or no deviation from its commanded path), depending on external and internal disturbances, under failure or abnormal conditions [7]. Moreover, the performance of those controllers might be critically affected by abnormal flight conditions. For this reason, it is important to study and understand what are the issues caused by abnormal flight conditions in order to avoid safety and performance problems during the mission.

Aircraft accidents are, in the majority, related to failures in the systems or subsystems of the aircraft that lead to misjudgments of command and control [8 and 9]. With UAVs, there are not as many redundancies as in a regular size aircraft, thus, failures in its systems or subsystems can more easily lead to a crash. Common sources of failures are actuators and sensors. These directly affect control surfaces that, under failures, have their dynamic and aerodynamic characteristics changed. Furthermore, coupling effects with different axes of rotation contributes 
to difficulties in controlling the aircraft. The higher the severity of the failure, the more difficult it is to maintain the aircraft stable and controllable.

Aircraft are constantly flying with the wind blowing in different directions, but wind direction and speed changes are usually gradual. The problem surges when the changes occur abruptly, which may lead to a catastrophic loss of control [10-12]. Wind phenomena are classified according to their duration, direction, and magnitude; and follow within different categories such as wind gusts, wind shear, constant wind, and turbulence. A sudden change in the direction of the wind during landing or takeoff, for example, can be catastrophic since the aircraft might hit the ground or miss the runaway and hit nearby buildings or trees.

Depending on the wind magnitude and period in which the aircraft is exposed to it, or the combination of wind phenomena with subsystems failures, the aircraft may lose altitude or be "blown" away (in the case of small UAVs) and be set far from its supposed course. Since the aerodynamics are sensitive with respect to the wind, the aircraft might not be able to recover from sudden or large interference. Therefore, it is crucial to understand how exactly the forces of the wind can affect the flight of UAVs to know how to properly address trajectory tracking controllers or make improvements in current available controllers.

Despite the importance of the matter in question, a detailed investigation of such flight environment for UAVs is scarce. Attempting to fulfill this need, this research effort was done by analyzing different flight path trajectories while the UAV is under upset conditions of various magnitudes of constant wind, turbulence, wind gust and wind shear.

\subsection{Research Objectives}

This research was conducted with the goal of investigating the effects of major atmospheric phenomena on the trajectory tracking performance of autonomous UAVs. Simplified models of wind were developed in order to study and understand its effects on two trajectory tracking control laws of distinct complexity (fixed parameter and adaptive parameter). The developed models of constant wind, wind gusts and wind shear were built in a Matlab/Simulink platform integrated with the WVU UAV simulation environment. A user friendly GUI (graphical user interface) facilitates implementations of different parameters and scenarios for the simulations. It allowed for different inputs of wind phenomena with specific direction, magnitude, duration and moment of occurrence. Performance of the controllers were calculated based on control surface activity and trajectory tracking errors. Additionally, aircraft upset 
conditions were induced along with the effect of wind phenomena to address the issue of combined effects when a control surface is not functioning.

\subsection{Thesis Outline}

This thesis contains 6 Chapters and is organized as follows. After this brief introduction, Chapter 2 contains literature review information on the different types of atmospheric phenomena, such as constant wind, turbulence, gusts, and wind shear. It also contains a brief discussion of aircraft abnormal conditions. The differences among all these phenomena, their effects on aircraft performance, and the danger associated with each one, are also addressed in this chapter. In Chapter 3, the modelling approach of constant wind, turbulence gusts and wind shear is described and the equations used are explained. Chapter 4 refers to the WVU UAV simulation environment, where the controllers considered in this thesis are described as well as the trajectories considered for the simulations. Chapter 5 describes the experiment design and respective factors and levels, along with results of the experiments and analysis of the controllers' sensitivity performance to wind phenomena. The conclusions regarding the effects of different wind phenomena and additional aircraft upset conditions, as well future research work recommendation are presented in Chapter 6. 


\section{LITERATURE REVIEW}

Modeling of wind phenomena involves a thorough understanding of the effects of the wind over the aircraft and how that will modify the dynamics of the aircraft. Thus, this chapter will introduce some approaches and considerations of atmospheric phenomena that need to be used for calculations of an accurate model that will most likely represent a real life application. Some dangers associated with the respective problems will be introduced and upset aircraft conditions concept will be briefly discussed.

\subsection{Aircraft Accidents Related To Wind and Failures}

The variability of wind has long been a concern for aircraft operation, it represents a potential hazard to aviation and needs to be carefully studied. There are several fatal and nonfatal accidents reported due to wind shear or turbulence [13]. Studies have been done on how pilots could avoid those phenomena, recover from them, and how much the wind shear or turbulence would affect flight in general. Aircraft accidents have also been linked with actuator failures where a control surface will lock and preclude the pilot from doing a specific maneuver. Depending on the time the failure occurred, this can lead to loss of control and a crash. Wind phenomena are very concerning, and if added to aircraft system failure, the consequences may be fatal.

It has been widely recognized that small downdrafts named microbursts are a serious hazard to aviation especially during landing or takeoff [13]. The Federal Aviation Administration conducted reports on aircraft accidents/incidents related to low altitude wind shear that occurred in the period of 1964 through 1975. It was found that more than 19,000 events happened during terminal area operations and only 25 involved large aircraft (more than 12,500 pounds) [13].

Investigation over the crash of the Pan American World Airways Flight 759 (Boeing 727) that occurred on July 9th, 1982 at the New Orleans International Airport, found that a microburst shortly after takeoff was responsible for the accident, killing all 145 on board and 8 people on the ground [14]. Another similar accident occurred on July 2nd, 1994 at Charlotte Douglas International Airport with the USAir Flight 1016. A thunderstorm was in progress and the crew encountered intensive wind shear inside a "wall of heavy rain" as described by Smith in [15]. Action to avoid the storm was not taken until it was too late and 37 people were killed. The same

unfortunate destiny had killed almost all of the passengers of the Eastern Airlines Flight 66 that 
crashed at John F. Kennedy airport in New York on June 24th, 1975. Adverse wind was blamed for the accident and 113 people perished [16]. Many other aircraft accidents related to wind shear occurred over the years. It was not until the "downburst" concept of Dr. Ted Fujita from the University of Chicago was finally accepted, that safety measures were incorporated and the number of accidents practically disappeared [17]. Pilots began to be trained to avoid and recover from such hazard and equipment to predict such type of weather was installed in many airports [17].

Between 1992 and 2001, there were 4,326 accidents related to weather conditions in the United States. During this period, 115 fatal accidents that resulted in 251 fatal injuries on board the aircraft, were cited as having turbulent weather as either a cause or a factor [18]. The Flight 587 of American Airways crashed shortly after takeoff due to turbulence encountered in New York in November 2001. Unnecessary use of the rudder by the crew in an attempt to compensate for the turbulence, led to the accident resulting in 265 fatalities [19]. On December 28th, 1997 a woman was killed, and 74 other were injured when the Flight 286 of United Airlines was hit by a severe turbulence over the Pacific. The jet was at 33,000 feet when the turbulence occurred. The aircraft dropped and the moving passengers and flight attendants were tossed. Several people were injured and one was killed [20].

Aircraft loss of control (LOC) accidents are a significant percentage of aviation fatalities regarding all classes and operations. $L O C$ is a result from contributing factors that happens individually or in combination. 126 LOC accidents occurred in the time range from 1979 to 2009 resulted in 6,087 fatalities [21]. Out of the 126 accidents, 42 or $33.3 \%$ were caused by system faults/failures or errors, which resulted in 1,544 fatalities.

There are innumerous incidents/ accidents related to subsystem failures. For example, an elevator failure of the McDonnel Douglas DC-8-71F during takeoff of Emerald Worldwide Flight 17, resulted in loss of pitch control on February 16th, 2000 in Rancho Cordova, California. The accident resulted in 3 casualties and the accident was addressed as improper maintenance. The right elevator got disconnected and resulted in loss of control of the pitch command [22]. Another accident involving improper maintenance occurred with Flight 261 of Alaska Airlines on Jan 31st, 2000. The trim system jackscrew assembly of the horizontal stabilator failed due to excessive wear resulting from insufficient lubrication. The aircraft crashed into the Pacific Ocean and left 88 people dead [21]. In October 1997 a fatal accident resulting from inappropriate use of control surfaces happened in Nuevo Berlin in Uruguay, with the Flight 2553 from Austral Lineas Aereas. The aircraft got into an uncontrolled descent due to extension of Slat/Flap by the crew, 
this resulted in an asymmetry due to the high speed and consequently loss of control of the vehicle [23].

Loss of control can also be associated with external hazards. As previously discussed, wind influences can lead to aircraft vehicle upset conditions and consequently loss of control. According to Belcastro and Foster [21], 86\% of flights that encountered thunderstorms, wind shear or gusts led to vehicle upset and moreover to incidents/accidents resulting in 84 fatalities. The opposite can also occur if the aircraft has already encountered an abnormal condition and subsequently encounters turbulence or wind shear. Among the data investigated, 228 fatalities were related to aircraft failure that encountered an external hazard [21]. Over the years, the number of accidents has been decreasing due to new technology and better preparation of the crew regarding these issues.

\subsection{Wind Phenomena Origin}

The atmosphere is a mixture of gases composed of nitrogen (78\%), oxygen (21\%) and others (1\%) and is divided into five layers (troposphere, stratosphere, mesosphere, thermosphere and exosphere). Most of the weather and clouds are found in the first layer [10 and 24]. The air can be characterized by pressure, density, and temperature. From those parameters, it is possible to specify some properties commonly used in aviation calculations such as: static pressure, dynamic pressure, total pressure, air density, and temperature. These thermodynamic parameters vary with altitude (vertically) and horizontally (due to weather) and in time (intrinsic dynamics) [10].

The weather is constantly changing throughout the world and the movement of the mass of air is the major factor of this change. The flow of gases in large scale is referred to as "wind", which is classified depending on its spatial scale, speed and region in which it occurs and its effects [25]. Velocity, density, and energy are the most important aspects related to wind. In meteorology, the classification of wind depends on its strength and direction. Short bursts of high speed wind are defined as gusts, while strong wind with intermediate duration is termed squall. Depending on the average strength, long-term wind can be classified as gale, breeze, storm, and hurricane.

There are three forces that add together to make wind: pressure gradient force, Coriolis force, and friction [12]. The Pressure gradient force works by trying to equalize differences in pressure, the high pressure pushes air towards low pressure. The second force is named after the French scientist Gustav-Gaspard Coriolis who discovered it in 1835 [26]. His principle states 
that "for a rotating body there is an inertial force acting on the body at right angles to its direction of motion; therefore, this forces results in a curved path for a body that would otherwise travel in a straight line". The Coriolis force determines the direction of wind and is responsible for the direction of hurricanes, tornados and spin direction of water draining from tub [27]. This principle is illustrated in Figure 1. The third force is friction, since the surface of the Earth is irregular, it causes the wind to diverge and slowdown.
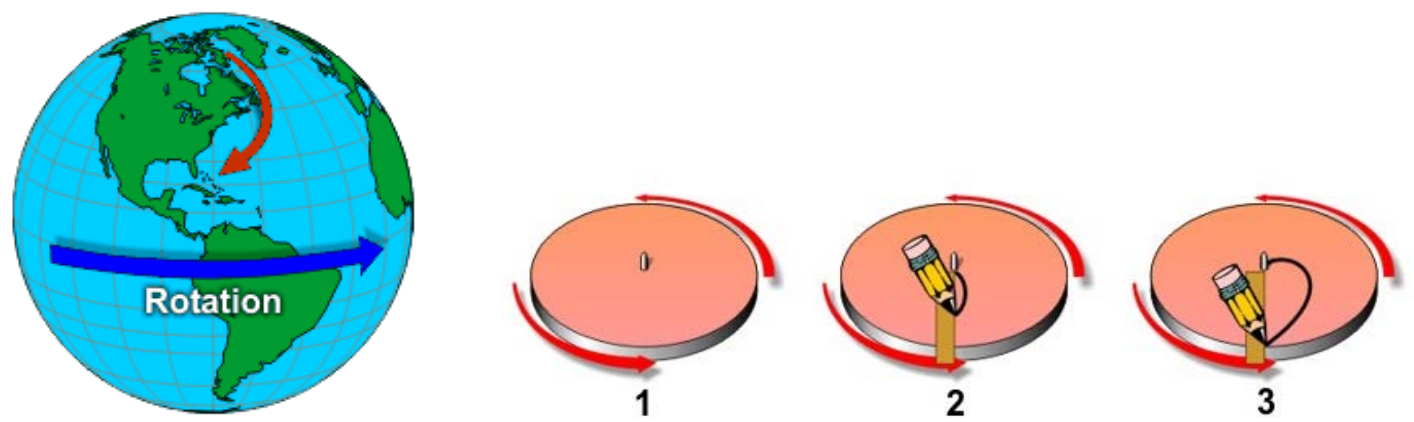

Figure 1 - Coriolis Force Effect on Earth Atmosphere and Rotating Surfaces [12].

During the day the air is heated by absorbing energy from the sun. Once it is hot, its pressure and density lowers, causing the air to raise and be substituted by cold air (higher pressure and density). Once the hot air is up, it cools down. This process makes the air pressure to rise again, thus, the cool air will lower eventually and force the hot air underneath to rise. This cycle continues repeatedly and this is how clouds and storms are originated. This process is illustrated in Figure 2 below.

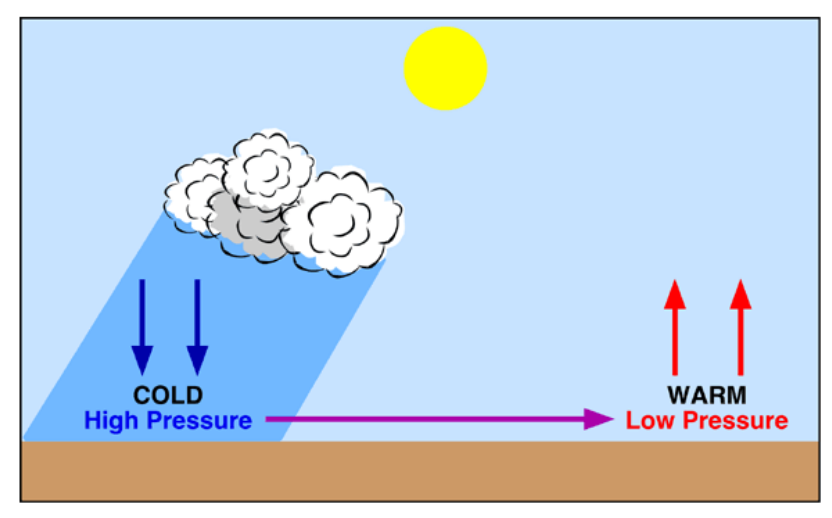

Figure 2 - How Wind Is Formed [28].

When an aircraft moves through the air, it generates aerodynamic forces such as lift that depends on the square of the velocity between the aircraft and the air. Since the aircraft is 
moving through the air, and the air itself is also constantly moving, it is important to define a fixed reference point in order to measure the velocities related to that point. The fixed point in the figure below is the aircraft, although it could have been fixed on the ground as well [29].

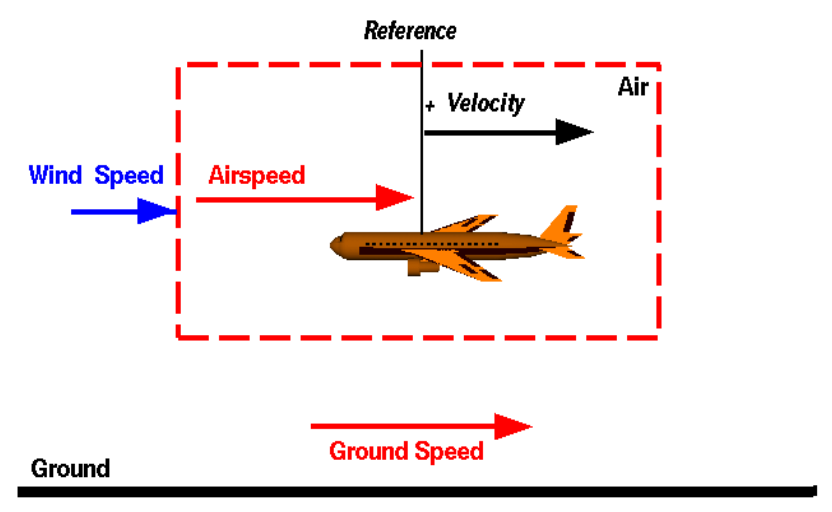

Figure 3- Relative Velocity and Wind Speed [29].

For a fixed reference point (aircraft), the air moves relative to this point at the airspeed. Furthermore, a positive wing velocity in the direction of the tail denominated "headwind" and a negative velocity moves towards the nose and is called "tailwind". The air moves in any direction, thus, the wind can be classified according to its direction. Updraft (away from the ground) and downdrafts (towards the ground) occur perpendicularly to the ground, and crosswinds (Figure 6) are perpendicular to the flight path but parallel to the ground [30]. The ground speed is defined as the ground velocity to a reference point picked on the aircraft, then, the wind speed can be finally calculated as the difference between the airspeed and the ground speed. For aircraft reference point, the wind velocity is considered positive, and for a ground reference it is considered negative. The following figure shows the vector addition of the two velocities discussed.

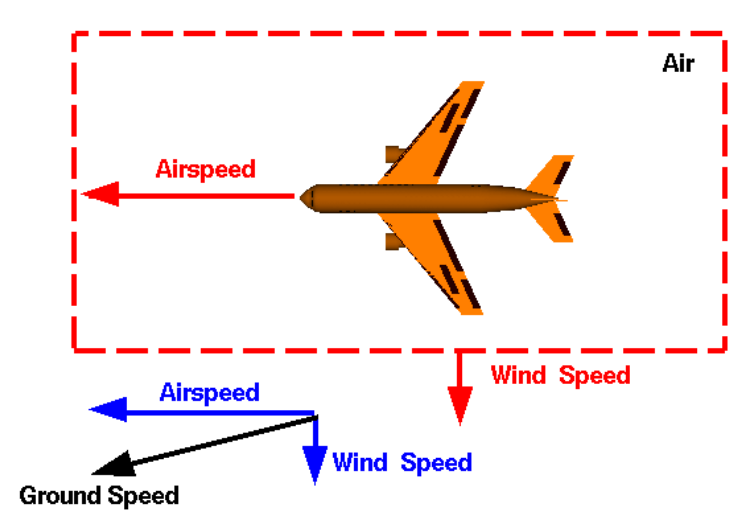

Figure 4 - Wind Speed Vector Addition [29]. 
The glide angle in the figure below is the angle formed by the flight path and the ground. Moreover, the airspeed can be decomposed into vertical and horizontal components. If the aircraft is descending the vertical component is negative, and it is positive for ascending flight. The vertical velocity can be found as the wind speed plus the vertical speed.

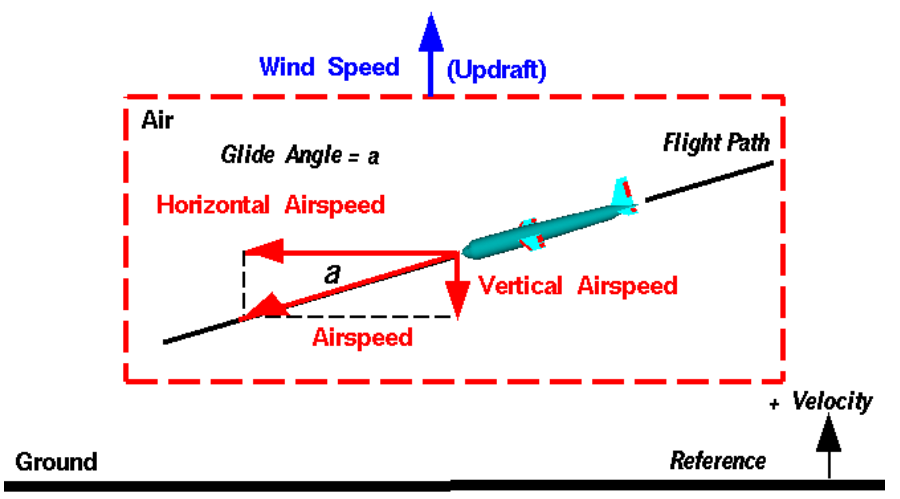

Figure 5- Vertical Velocity [29].

If the vertical component of the airspeed is smaller than the updraft, the aircraft will gain altitude. On the other hand, for a strong downdraft, the aircraft may lose altitude even in a climb. Downdraft is mostly found in the vicinity of thunderstorms and is associated with the movement of cold air mass [29 and 31]. Downdrafts near airports can cause the aircraft to lose altitude and crash, Figure 7 illustrates updraft and downdraft formation. Since aircraft are aerodynamic, wind affects its flight speed as well as ground speed. Thus, the direction of flight operations (takeoff and landing) are based on the direction of the local wind at airports [25 and 29]. Furthermore, runaways are designed to have the same direction of the local wind, to ensure safety while takeoff and landing. Headwind is desired during takeoff while tailwind can increase takeoff, distance and decrease climb gradient.

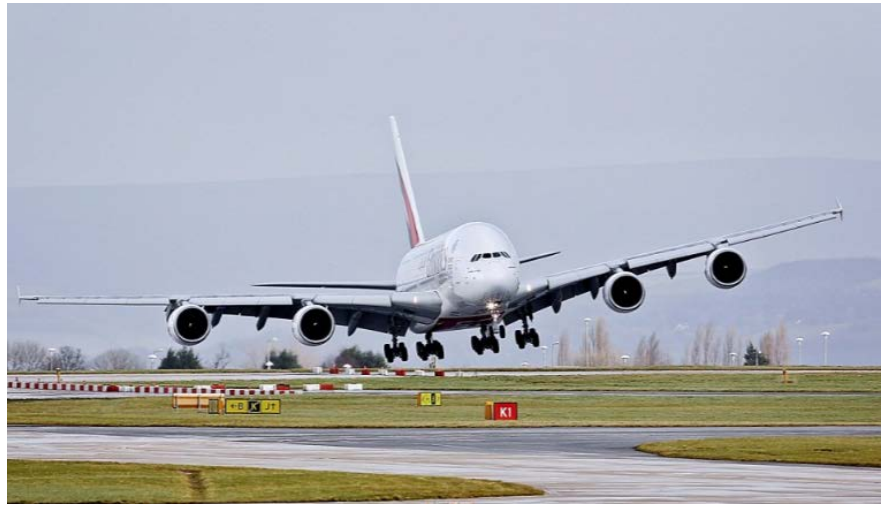

Figure 6 - Aircraft A380 Experiencing 60 mph Crosswind During Landing [32]. 


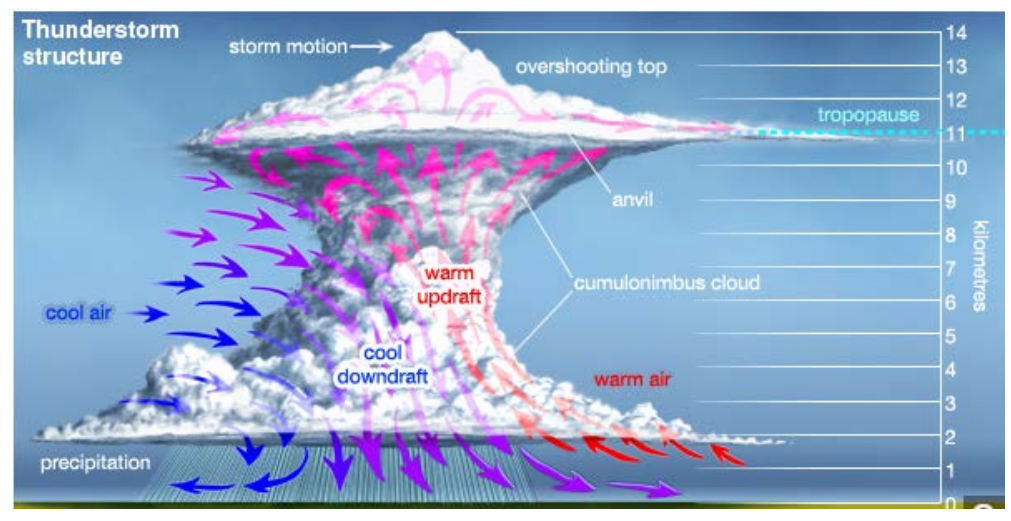

Figure 7- Downdraft and Updraft Sketch [31].

Following the same principles as previously discussed, a common wind phenomenon that is extremely important in aviation is wind gust. According to Weather Guys, a gust is "a sudden, brief increase in the speed of the wind followed by a lull" [33]. It is generally reported when the speed of the wind reaches about 16knots, and the variation in between the highest peak speed and lull is more than 9 knots. A gust is the highest wind speed reached and lasts less than 20 seconds [33 and 34]. The effect of wind gusts during landing can be seen in Figure 8 below. The air carried down strikes the ground and spreads out in shallow layers.

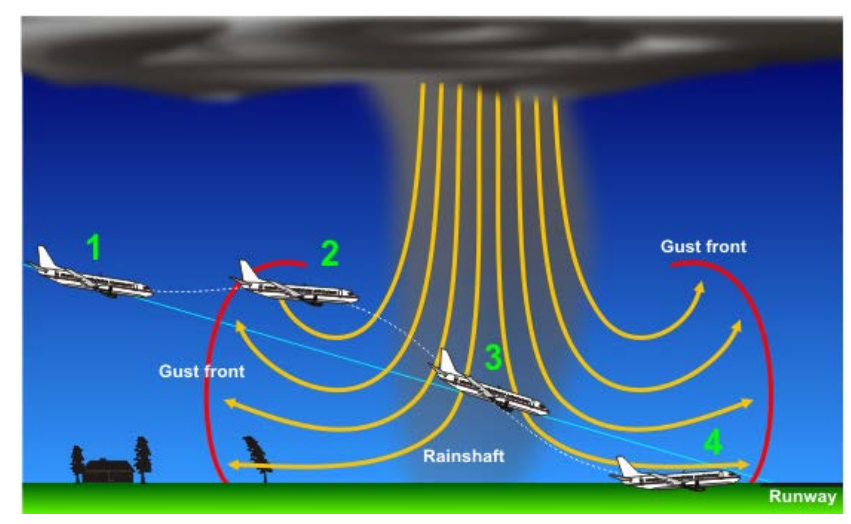

Figure 8- Wind Gusts Effects on Aircraft Flight Path [35].

While wind gusts affect aircraft flight on a daily basis, publications on automatic control of UAVs under wind gusts are scarce. In the article published by Leonard et al. [36], a controller for a helicopter drone was developed to perform under wind gusts. An attempt to counteract the effects of gusts (vertical and lateral only) was done using a 7-degrees-of-freedom nonlinear Lagrangian model with two robust controllers (non-linear feedback and active disturbance rejection control). Furthermore, a technique to guarantee steady state flight under specified probability when stochastic wind gusts occurs was developed by Richardson et al. [37]. In this 
technique, the gust is treated as a stationary random process and is used as input. The dynamic equations were perceived as a linear time invariant system with the airplane velocity and angular velocity as the state. Moreover, the Dryden and Karman models were applied in combination to allow a development of a tool to analyze how the gusts affects steady flight. Another example of relevant and similar work was done by Raza [38], for position control of a quadrotor UAV in an urban environment. In this study, the wind gusts were modelled from computational fluid dynamics using a large eddy simulation. Moreover, another research was done by Gaonkar [39], in which gusts excitations are nonstationary and are approximately Gaussian, and the flight regimes for certain vehicles are linear. Thus, Rice's equations can be applied to evaluate threshold crossing and peak statistics. Gusts excitation can be reasonably idealized as separable, nonstationary process and the stationary excitation is modulated by a deterministic function.

Changes in the wind direction and magnitude are a hazard and a challenge for aviation and automatic control of small UAVs. One of the most challenging wind phenomena to deal with is wind shear. It is defined as a change in the wind speed and/or direction over a short distance [40]. Wind shear is measured by dividing the velocity difference at two points by the distance in between them [13]. It can occur vertically or horizontally, at low or high altitude and is associated with temperature inversions and density gradients. A vertical wind shear is described as a change in wind speed or direction with changes in altitude, and a horizontal wind shear is a change in wind speed over a lateral/longitudinal position for a certain altitude [41]. The most concerning hazard to aircraft occurs horizontally in distances of 1 to 10 miles [13]. The most dangerous type of wind shear is low-altitude wind shear because there may not be enough altitude to recover before impacting terrain. Wind shear is mostly a result from various meteorological situations such as topographical conditions, temperature inversions and in its most violent forms, thunderstorms and rain shower [42]. Based on the study done by Rogers et al. [43], the direction and speed of the wind has an effect on the wind shear, high wind shear occurs more frequently at lower wind speeds.

A microburst (powerful downdraft) is considered the most dangerous type of wind shear. Whenever an aircraft encounters a microburst in its initial stage, its effects may be insignificant at first, but later on, the airplane may experience an airspeed change of two to three times greater than before encountering wind shear. Measurements from Doppler radar, indicates is most likely that the highest average speed that an aircraft may encounter is 45 knots. However, microbursts of about 200 knots have been measured [42]. Figure 9 and Figure 10, represent the hazard of encountering wind shear during takeoff and landing. 


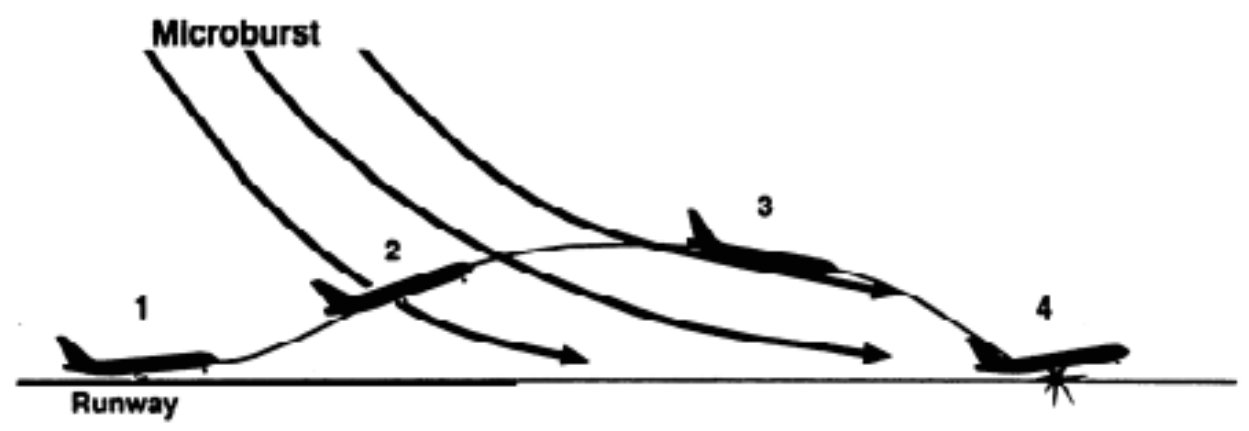

Figure 9- Wind Shear Encounter During Takeoff After Liftoff [42].

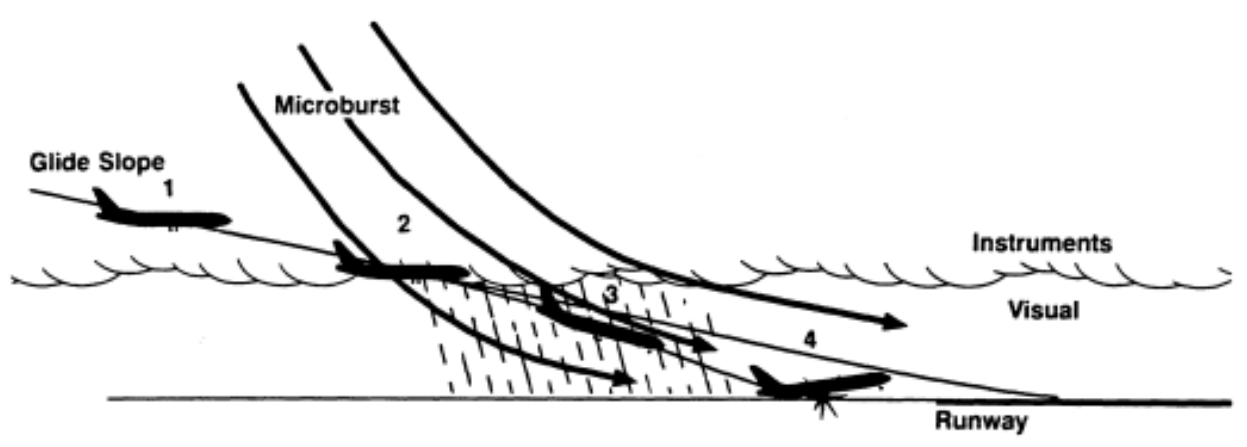

Figure 10- Wind Shear Effect During Landing [42].

Only 5 to 15 seconds may be available to recognize and take actions in response to a wind shear encounter according to Beaudette [42]. To address and study wind shear, some research and modelling were done in previous work, as found in [44], where investigation of the dynamics of an aircraft was done under wind shear of arbitrary direction. In that paper, a solution for steady-state is defined, then investigated using a linear system about the solution. The conclusion is that wind shear affects coupling of the longitudinal and the lateral motions by producing an additional mode. In [45], the author addresses the variables of wind terms into the equations of motion, including temporal and spatial gradients of wind. Moreover, effects of the wind shear inputs in computing aerodynamics coefficients and wind velocity vector rotation effect on relative angular rates of rotation are also addressed. This model approach however, is later corrected in [46], where Etkin suggests that this model must consider the extra rolling moment term associated with the wind gradient in some of the equations.

A relevant approach to estimate the state of the aircraft under nonlinear longitudinal motion was done by Mulgund et al. [47]. In that study, an Extended Kalman Filter (EKF) was 
developed to provide estimates of horizontal and vertical atmospheric wind inputs. The disturbance and state estimate were incorporated in feedback control, based on the aircraft's nonlinear inverse dynamics. The EKF is able to accurately produce estimates of the aircraft trajectory. Based on the idea of EKF, an on-line identification of wind shear for UAVs has been proposed in [48]. The identification problem was solved by using filter error approach and the EKF was used to propagate the state. The wind shear effects were modeled as external forces and moments applied to the aircraft. Later, the algorithm was tuned by using a database of measurements through off-line identification of the process noise covariance matrix. Moreover, the EKF was used to estimate on board aircraft state or turbulence and demonstrated significant savings in terms of time and computing resources.

Changes in pressure and flow velocity are the origin of turbulence, which can be defined as nature going to the state of maximum disorder [49 and 50]. The effects of turbulence ranges from jostling the aircraft, to sudden accelerations that can result in temporary loss of aircraft control [51]. Generally, turbulence is associated with visible storms. Although, clear air turbulence can even be found nearby thunderstorms up to 50 miles away from the actual storm [52]. Clear air turbulence occurs at regular cruise altitudes with, usually, no visible warning for the pilots. Furthermore, it can also be formed nearby high altitude air currents, in the vicinity of mountains and weather fronts. Effects of turbulence can be aggravated in weather conditions involving wind shear, where the turbulence masks the changing airspeed trends and delays the detection of severe wind shear [42]. Turbulence is originated by excessive kinetic energy in fluid flows. This excess in energy overcomes the damping of the fluid viscosity, therefore, turbulence is easier to form in fluids with low viscosity. Drag increases resulting from the interaction of the vortices with one another, becoming a problem for aircraft because it increases fuel consumption.

In order to model turbulence, two most common models available are the Dryden and Von Karman wind turbulence model. According to Beal, both models define the power spectra analytically with three velocity spectra, related to the three axes associate with body coordinated system [53]. The main difference is that with the Dryden model an exact filter can be designed that takes the white noise inputs and outputs a random process with the model's power spectral densities. With the Von Karman model, the filter designed can be only approximated.

Studies of how turbulence (at few meters above ground) can influence microair vehicles was done by Waltkins et al. [54]. Transient pitch flows were investigated and it was found that the variation with the lateral separation of 4 pressure probes placed 150 and $50 \mathrm{~mm}$ apart 
decreased slowly with reducing separation. The pitch angle and the effect with lateral separation may be described non dimensionally. Furthermore, a parametric computational aerodynamics (PCA) model was compared by Staveren, to two classic models, the Delft University of Technology model and the Four-Point-Aircraft model. The purpose was to investigate the response of an aircraft to stochastic atmosphere turbulence [55]. These models can be used to calculate time and frequency domain aerodynamic model and the aircraft responses to atmospheric turbulence. It was concluded that the PCA is the most accurate, especially for the aircraft response to $2 \mathrm{D}$ gust fields.

\subsection{Aircraft Abnormal Conditions}

Abnormal or upset condition is any condition in which the aircraft is not operating with all systems and conditions proper to safe flight, as designed. These conditions may play a major role in loss of control and aircraft accidents. Intelligent on board monitoring systems may accurately detect and identify aircraft upset conditions as soon as they take place in order to be able to correct for any discrepancy and be able to maintain the safe control of the aircraft. Among several different abnormal conditions that the aircraft could encounter during flight, a major concern is represented by subsystem failures [56]. For instance, actuator failures are often critical for aviation safety, thus, failures regarding elevator, aileron and rudder need relevant attention since they directly affect the control of the aircraft. The main characteristics of each failure is how much each of the control surfaces affects its axis of motion. Furthermore, each surface failure has a different dynamic fingerprint and affects the aircraft moments differently [57].

For a stabilator or elevator lockage, the aircraft experiences a coupling moment between the lateral and longitudinal axes. The aircraft will experience a roll-pitch effect and depending on its severity it may even cause a slight yaw moment. For example, if the right stabilator fails with a positive deflection (up), the nose will pitch down and there will be rolling to the left and a slight yaw to the right (see Figure 11). The respective effects increase as the severity of the failure increases. A failure of the stabilator will affect primarily the pitch and roll moments.

A failure of the ailerons will affect mostly the rolling moment of the aircraft. The failure has a small yaw moment associated with it as well, since is located on the trailing edge of the wing. A deflection of the ailerons will cause the aircraft to roll left or right depending on the degree of deflection and side of the wing. For example, a positive deflection on the right aileron (deflected downwards) will result in lift on the right side of the wing and cause the aircraft to roll to the left. This dynamic response increases as the deflection of lockage increases (see Figure 12). 
When a lockage failure occurs on the rudder, for single rudder aircraft, it can be difficult to compensate for the asymmetry in the aerodynamics. In this case, the yaw and roll moments will be primarily affected. A rudder failure with positive deflection (deflected to the left) will cause the aircraft to yaw to the left and roll to the left or right depending on the coupling. The angular rates will depend on the rudder deflection (see Figure 13).

In general, failures in actuators that command different control surfaces will affect primarily their axis of control. However, some coupling may occur depending on the severity of the failure, which gives each failure its characteristic dynamic fingerprint.

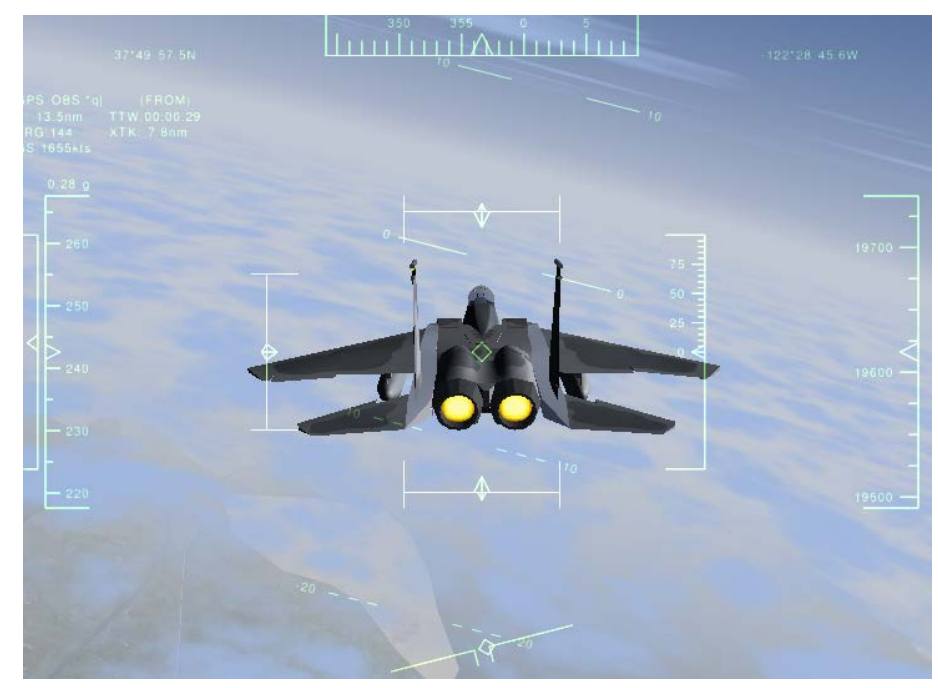

Figure 11- Right Stabilator With Positive Deflection.

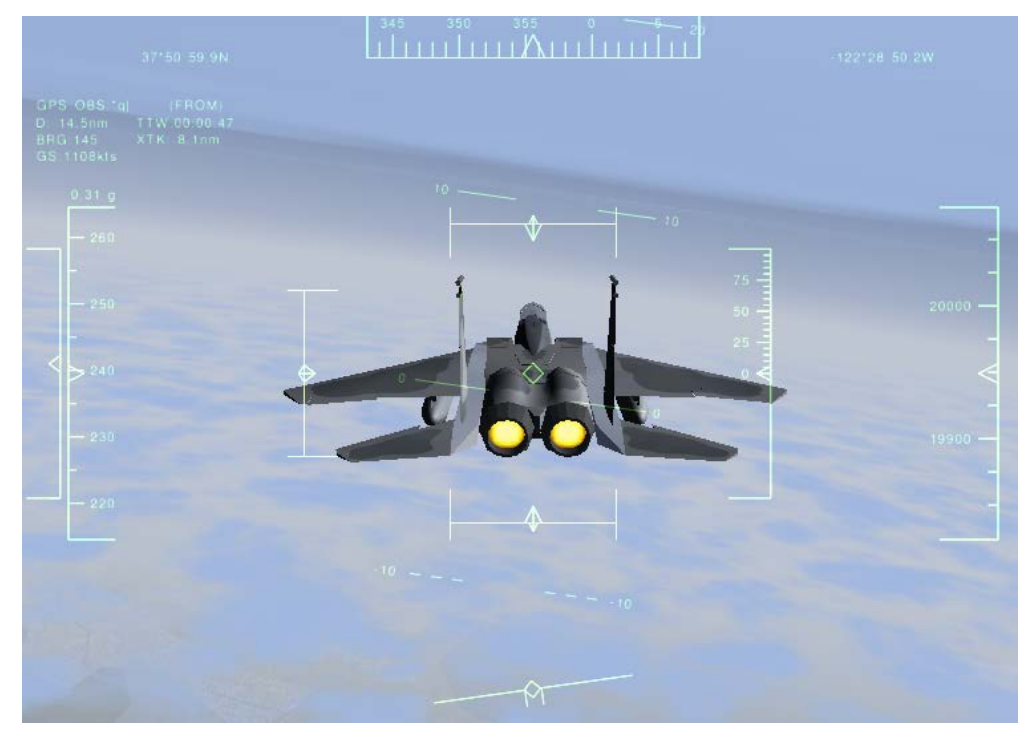

Figure 12- Right Aileron With Positive Deflection. 


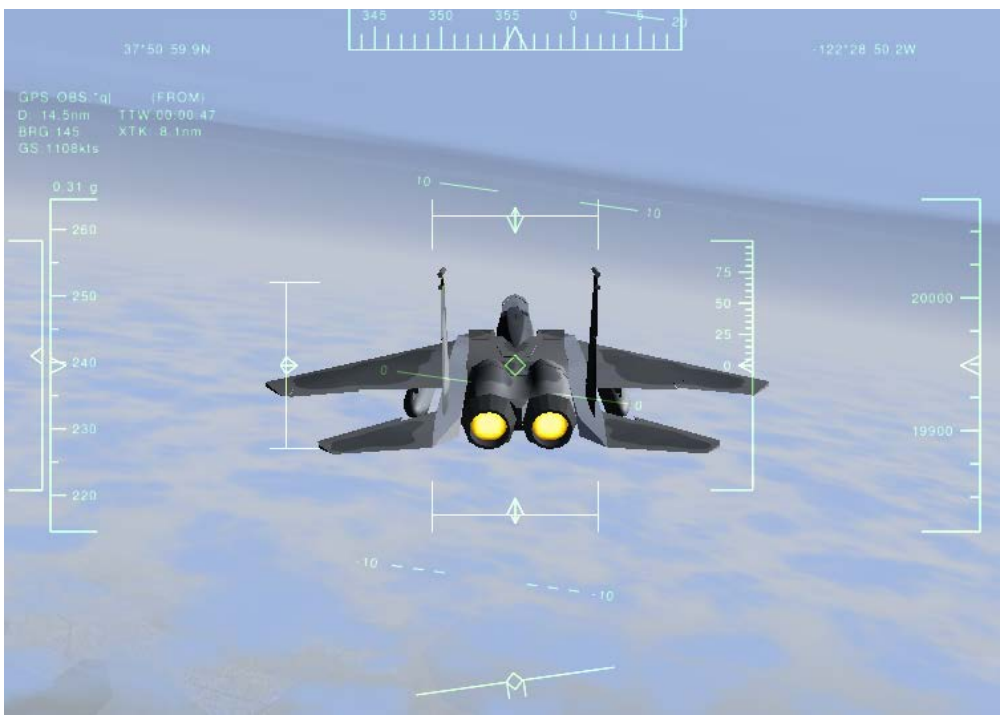

Figure 13-Right Rudder Positive Deflection. 


\section{WIND MODEL}

\subsection{Definitions and Notation}

\subsubsection{Reference Frame}

A reference frame (RF) is a set of points whose distances to each other are constant. In the Euclidean 3-dimensional space, a reference frame can be defined by a minimum of 4 noncollinear points that do not move with respect to each other. RFs are often used to define physical quantities, such as velocity of a point with respect to the RF. A RF that is considered to move at 0 acceleration is called an inertial RF.

\subsubsection{Coordinate System}

The position of any point with respect to a RF is defined based on a coordinate system (CS) associated to the RF. A CS in the Euclidean 3-dimensional space is a set of 4 points that belong to the RF. One of them is selected as the origin and is used to define the CS axes along the three segments that connect the origin to the remaining 3 points. These 3 points are typically selected such that the three CS axes are mutually perpendicular, thus defining a Cartesian CS. A multitude of CSs may be associated to any given RF. For a particular $R F_{E}$, an associated CS will typically be denoted as $C S_{E}$ or by explicitly listing its origin and the indicatives of the three axes: $E X_{E} Y_{E} Z_{E}$. The positive directions of the three axes must be specified to completely define the CS [58].

\subsubsection{Rigid Body}

A rigid body is a set of particles or material points that do not move with respect to each other. Very often it is convenient to associate a RF to a rigid body. A particle or a material point is a point that possesses mass.

\subsubsection{Position Vector}

The position vector of a point $C$ with respect to another point $O$ is denoted as $\vec{r}^{O C}$. The position vector originates at point $O$ and is directed towards $C$.

\subsubsection{Velocity Vector}

The velocity $V$ of a point $C$ with respect to a reference frame $R F_{E}$ is denoted as ${ }^{E} \vec{V}^{C}$. Furthermore:

$$
{ }^{E} \vec{V}^{C}=\frac{{ }^{E} d \vec{r}^{O C}}{d t}
$$


where $\frac{E_{d}}{d t}$ is the derivation operator with respect to $R F_{E}$. Note that $O$ is any fixed point in $R F_{E}$ [59].

\subsubsection{Vector Coordinates}

The coordinates of vector $\vec{V}$ with respect to $C S_{E}$ (or $E X_{E} Y_{E} Z_{E}$ ) are denoted as:

$$
[\vec{V}]_{E}=\left[\begin{array}{c}
V_{x} \\
V_{y} \\
V_{z}
\end{array}\right]_{E}
$$

\subsubsection{Angular Velocity Vector}

The angular velocity of a rigid body $B$ with respect to reference frame $R F_{E}$ is denoted as: ${ }^{E} \vec{\omega}^{B}$. Note that angular velocity is instrumental in establishing the relationship between the timederivative of a vector $\vec{V}$ with respect to a reference frame $R F_{E}$ and the time-derivative of the same vector with respect to a different reference frame $R F_{B}$ :

$$
\frac{{ }^{E} d \vec{V}}{d t}=\frac{{ }^{B} d \vec{V}}{d t}+E \vec{\omega}^{B} \times \vec{V}
$$

where rigid body $B$ is fixed in $R F_{B}$.

\subsubsection{Euler Angles}

The relative orientation of two CSs is defined by three Euler or attitude angles. They are obtained through three successive rotations along one axis at a time applied to one CS, such that it eventually overlaps the second. For an aircraft, these angles represent the orientation of the fixed body axes with respect to a CS fixed with respect to the Earth [58]. They are referred to as aircraft attitude angles and denoted as roll, pitch, and yaw attitude angles $(\varphi, \theta, \psi$, respectively). The typical order of axes rotation is vertical first followed by lateral and longitudinal. With most commonly used conventions, the roll attitude angle is positive if the aircraft is tilted to the right of the pilot, the pitch attitude angle is positive if the aircraft is tilted nose-up, and the yaw attitude angle is positive if the nose of the aircraft points to the right of the pilot (Figure 14). 


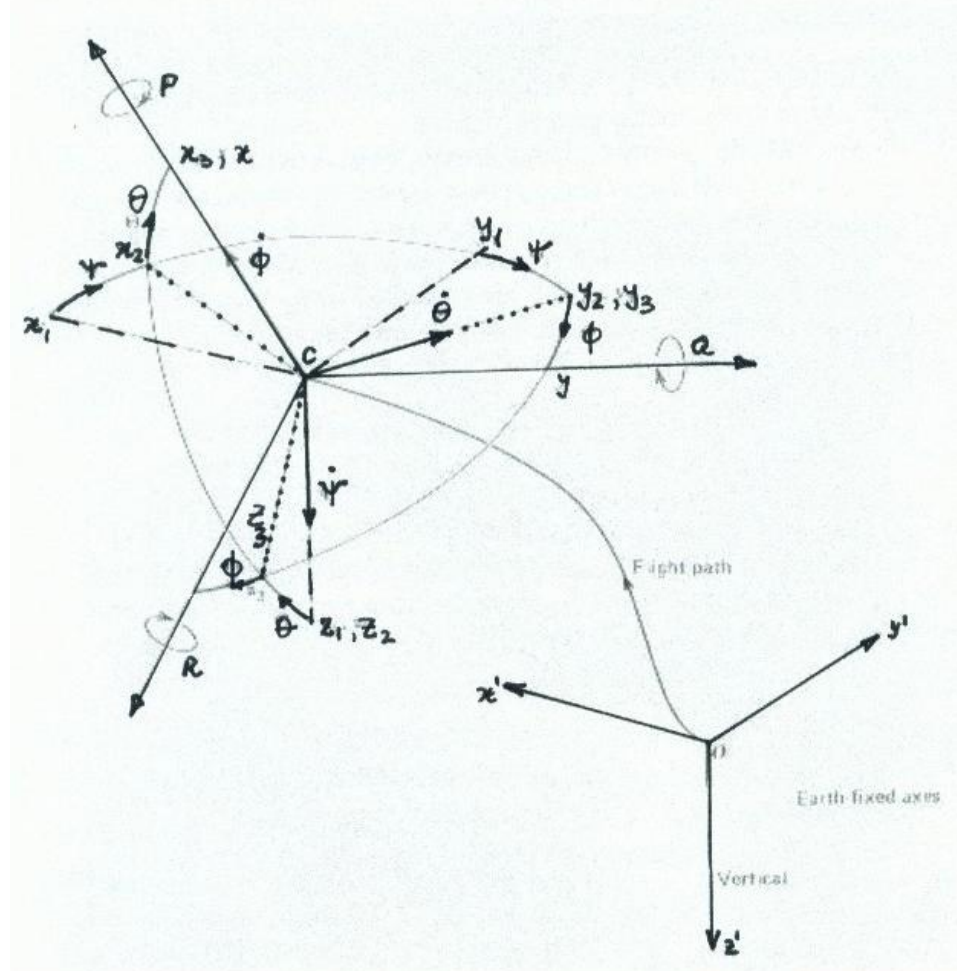

Figure 14- Relative Position of the Body and Earth CS [58].

\subsubsection{Transformation Matrix}

The transformation matrix from $C S_{E}$ to $C S_{B}$ is denoted as $L_{B E}$. The transformation matrix allows the computation of vector components or coordinates with respect to one CS when the values with respect to another CS are given. That is:

$$
[\vec{V}]_{B}=L_{B E}[\vec{V}]_{E}=L_{E B}^{-1}[\vec{V}]_{E}
$$

Note that transformation matrices are orthonormal. Therefore: $L_{B E}^{-1}=L_{B E}^{T}$. The elements of the transformation matrices are trigonometric functions of the Euler angles between the two CS.

\subsection{Reference Frames and Coordinate Systems}

\subsubsection{Earth Frame and Coordinate System}

The Earth is assumed to be flat and inertial. A working CS relative to the Earth is denoted $C S_{E}$ or $E X_{E} Y_{E} Z_{E}$. Within the WVU UAV Simulation Environment, the origin $E$ is established by the user on the interactive map and coincides with the initial location of aircraft center of mass. The longitudinal Earth axis $X_{E}$ is selected to point up (towards North) with respect to the displayed map. The lateral axis $Y_{E}$ is positive to the right (Eastward). The vertical Earth axis $Z_{E}$ is positive into the plane of the map. 


\subsubsection{Aircraft Frame and Coordinate System}

The aircraft is assumed to be a rigid body with constant mass. The aircraft CS, also referred to as "body axes", is denoted as $C S_{B}$ or $C X_{B} Y_{B} Z_{B}$. The origin is at the center of mass of the aircraft. The longitudinal axis is along the fuselage, positive forward, in the aircraft plane of symmetry, with a direction at the discretion of the designer. The lateral axis $Y_{B}$ is positive to the right of the pilot and the vertical axis $Z_{B}$ is positive downward, as dictated by the right hand rule [58].

The relative orientation of the aircraft $\left(C S_{B}\right)$ with respect to Earth $\left(C S_{E}\right)$ is defined by the Euler angles denoted as $\varphi, \theta$, and $\psi$. The transformation matrices between the two CS are:

$$
L_{B E}=\left[\begin{array}{ccc}
\cos (\psi) \cos (\theta) & \sin (\psi) \cos (\theta) & -\sin (\theta) \\
\cos (\psi) \sin (\theta) \sin (\varphi)-\sin (\psi) \cos (\varphi) & \sin (\psi) \sin (\theta) \sin (\varphi)+\cos (\psi) \cos (\varphi) & \cos (\theta) \sin (\varphi) \\
\cos (\psi) \sin (\theta) \cos (\varphi)+\sin (\psi) \sin (\varphi) & \sin (\psi) \sin (\theta) \cos (\varphi)-\cos (\psi) \sin (\varphi) & \cos (\theta) \cos (\varphi)
\end{array}\right]
$$

and

$$
L_{E B}=\left[\begin{array}{ccc}
\cos (\psi) \cos (\theta) & \cos (\psi) \sin (\theta) \sin (\varphi)-\sin (\psi) \cos (\varphi) & \cos (\psi) \sin (\theta) \cos (\varphi)+\sin (\psi) \sin (\varphi) \\
\sin (\psi) \cos (\theta) & \sin (\psi) \sin (\theta) \sin (\varphi)+\cos (\psi) \cos (\varphi) & \sin (\psi) \sin (\theta) \cos (\varphi)-\cos (\psi) \sin (\varphi) \\
-\sin (\theta) & \cos (\theta) \sin (\varphi) & \cos (\theta) \cos (\varphi)
\end{array}\right] \text { (6) }
$$

\subsubsection{Wind Frame and Coordinate System}

The mass of air is considered as a rigid body that translates at constant velocity $\vec{W}_{c}$ (constant wind) with respect to $R F_{E}$. A coordinate system is associated to this rigid body denoted as $C S_{W}$ or $O X_{W} Y_{W} Z_{W}$. The origin may coincide with the origin of $C S_{E}$ and its longitudinal axis is along the velocity vector $\vec{W}_{C}$. The relative orientation of $C S_{W}$ with respect to $C S_{E}$ is defined by three wind Euler angles $\varphi_{W}, \theta_{W}$, and $\psi_{W}$. Note that $\varphi_{W}=0$ in all instances. Therefore the transformation matrices with respect to Earth axes are:

$$
\begin{aligned}
L_{W E} & =\left[\begin{array}{ccc}
\cos \left(\psi_{W}\right) \cos \left(\theta_{W}\right) & \sin \left(\psi_{W}\right) \cos \left(\theta_{W}\right) & -\sin \left(\theta_{W}\right) \\
-\sin \left(\psi_{W}\right) & \cos \left(\psi_{W}\right) & 0 \\
\cos \left(\psi_{W}\right) \sin \left(\theta_{W}\right) & \sin \left(\psi_{W}\right) \sin \left(\theta_{W}\right) & \cos \left(\theta_{W}\right)
\end{array}\right] \\
L_{E W} & =\left[\begin{array}{ccc}
\cos \left(\psi_{W}\right) \cos \left(\theta_{W}\right) & -\sin \left(\psi_{W}\right) & \cos \left(\psi_{W}\right) \sin \left(\theta_{W}\right) \\
\sin \left(\psi_{W}\right) \cos \left(\theta_{W}\right) & \cos \left(\psi_{W}\right) & \sin \left(\psi_{W}\right) \sin \left(\theta_{W}\right) \\
-\sin \left(\theta_{W}\right) & 0 & \cos \left(\theta_{W}\right)
\end{array}\right]
\end{aligned}
$$




\subsubsection{Wind Shear Frame and Coordinate System}

For modeling wind shear, the wind CS will be translated at a point $S$, but orientation is identical. Therefore, this CS will be denoted as $C S_{S}$ or $S X_{W} Y_{W} Z_{W}$ and the transformation matrices will be $L_{S E}=L_{W E}$ and $L_{E S}=L_{E W}$.

Note that point $S$ is the point where the aircraft enters the wind shear zone and the spatial wind gradient is non-zero as described later.

\subsubsection{Gust Frame and Coordinate System}

Wind gust RF and CS are defined in a similar way as for the constant wind, but in association with the gust direction. Therefore, the orientation of $C S_{G}$ (or $O X_{G} Y_{G} Z_{G}$ ) with respect to $C S_{E}$ is defined by two Euler angles, $\theta_{G}$ and $\psi_{G}$ and the transformation matrices are.

$$
\begin{gathered}
L_{G E}=\left[\begin{array}{ccc}
\cos \left(\psi_{G}\right) \cos \left(\theta_{G}\right) & \sin \left(\psi_{G}\right) \cos \left(\theta_{G}\right) & -\sin \left(\theta_{G}\right) \\
-\sin \left(\psi_{G}\right) & \cos \left(\psi_{G}\right) & 0 \\
\cos \left(\psi_{G}\right) \sin \left(\theta_{G}\right) & \sin \left(\psi_{G}\right) \sin \left(\theta_{G}\right) & \cos \left(\theta_{G}\right)
\end{array}\right] \\
L_{E G}=\left[\begin{array}{ccc}
\cos \left(\psi_{G}\right) \cos \left(\theta_{G}\right) & -\sin \left(\psi_{G}\right) & \cos \left(\psi_{G}\right) \sin \left(\theta_{G}\right) \\
\sin \left(\psi_{G}\right) \cos \left(\theta_{G}\right) & \cos \left(\psi_{G}\right) & \sin \left(\psi_{G}\right) \sin \left(\theta_{G}\right) \\
-\sin \left(\theta_{G}\right) & 0 & \cos \left(\theta_{G}\right)
\end{array}\right]
\end{gathered}
$$

\subsection{Aircraft Equations of Motion}

With the rigid body assumption, the motion of the aircraft is typically modeled as a set of 4 first order non-linear differential vector equations. Two of them are dynamic equations, also referred to as force and moment equations and two are kinematic equations, also referred to as attitude (or rotation) and trajectory (or translation) equations. In vector components with respect to body axes and Earth axes these equations result in 12 first order non-linear differential scalar equations [58].

Four state vectors must be known in order to completely determine the motion of an aircraft as a rigid body:

$\vec{r}^{E C}=$ position vector of aircraft center of mass $C$ with respect to the origin $E$ of the fixed Earth CS, which defines the trajectory;

${ }^{E} \vec{V}^{C}=$ velocity of aircraft center of mass $C$ with respect to the fixed Earth reference frame, also referred to as ground speed; 
$\left[\begin{array}{lll}\varphi & \theta & \psi\end{array}\right]^{T}=$ orientation of body axes $C S_{B}$ with respect to Earth axes $C S_{E} ;$

${ }^{E} \vec{\omega}^{B} \quad=$ angular velocity of aircraft rigid body with respect to $R F_{E}$.

\subsubsection{Force Equations}

The linear momentum theorem provides the first vector equation. The time derivative with respect to the inertial reference frame of the linear momentum of aircraft center of mass $C$ with respect to the inertial frame is equal to the sum of all external forces acting on the rigid body.

$$
\frac{{ }^{E} d\left({ }^{E} \vec{H}^{C}\right)}{d t}=\sum_{i} \vec{F}_{i}
$$

Considering that:

$$
{ }^{E} \vec{H}^{C}=m^{E} \vec{V}^{C}
$$

where $m$ is the mass of the aircraft, for constant mass, this equation becomes:

$$
m \frac{{ }^{E} d\left(\vec{V}^{C}\right)}{d t}=\sum_{i} \vec{F}_{i}
$$

or:

$$
m\left(\frac{{ }^{B}\left({ }^{E} \vec{V}^{C}\right)}{d t}+{ }^{E} \vec{\omega}^{B} \times{ }^{E} \vec{V}^{C}\right)=\sum_{i} \vec{F}_{i}
$$

With standard notations for vector components, the scalar force equations result:

$$
\left[\begin{array}{c}
\dot{u} \\
\dot{v} \\
\dot{w}
\end{array}\right]_{B}+\left[\begin{array}{ccc}
0 & -r & q \\
r & 0 & -p \\
-q & p & 0
\end{array}\right]_{B}\left[\begin{array}{l}
u \\
v \\
w
\end{array}\right]_{B}=\frac{1}{m}\left[\begin{array}{c}
F_{x} \\
F_{y} \\
F_{z}
\end{array}\right]_{B}
$$

where:

$$
\begin{aligned}
{\left[{ }^{E} \vec{V}^{C}\right]_{B} } & =\left[\begin{array}{lll}
u & v & w
\end{array}\right]_{B}^{T} \\
\left.{ }^{E} \vec{\omega}^{B}\right]_{B} & =\left[\begin{array}{lll}
p & q & r
\end{array}\right]_{B}^{T} \\
{\left[\sum_{i} \vec{F}_{i}\right.} & ]_{B}=[\vec{F}]_{B}=\left[\begin{array}{lll}
F_{x} & F_{y} & F_{z}
\end{array}\right]_{B}^{T}
\end{aligned}
$$

\subsubsection{Moment Equations}

The angular momentum theorem provides the second vector equation. The time derivative with respect to the inertial reference frame of the angular momentum of the aircraft with respect to the inertial frame and the center of mass $C$ is equal to the sum of all external moments with respect to $C$ acting on the rigid body. 


$$
\frac{E_{d\left({ }^{E C} \vec{K}^{B}\right)}}{d t}=\sum_{i} \vec{M}_{i}^{C}
$$

Considering that:

$$
{ }^{E C} \vec{K}^{B}=\underline{I}^{B / C} \cdot E \vec{\omega}^{B}
$$

where $\underline{I}^{B / C}$ is the inertia tensor of $B$ with respect to its center of mass $C$, for constant inertia, the moment vector equation becomes:

$$
\underline{I}^{B / C} \frac{{ }^{E} d\left({ }^{E} \vec{\omega}^{B}\right)}{d t}=\sum_{i} \vec{M}_{i}^{C}
$$

With usual notations, the components of the resultant moment in body axes are:

$$
\left[\sum_{i} \vec{M}_{i}^{C}\right]_{B}=\left[\begin{array}{lll}
M_{x} & M_{y} & M_{z}
\end{array}\right]_{B}^{T}
$$

For symmetric aircraft, the components of the inertia tensor are:

$$
\left[\underline{I}^{B / C}\right]_{B}=\left[\begin{array}{ccc}
I_{x x} & 0 & I_{x z} \\
0 & I_{y y} & 0 \\
I_{z x} & 0 & I_{z z}
\end{array}\right]_{B}
$$

Therefore, the three scalar moment equations can be written as:

$$
\left[\begin{array}{ccc}
I_{x x} & 0 & I_{x z} \\
0 & I_{y y} & 0 \\
I_{z x} & 0 & I_{z z}
\end{array}\right]_{B}\left[\begin{array}{c}
\dot{p} \\
\dot{q} \\
\dot{r}
\end{array}\right]_{B}+\left[\begin{array}{ccc}
0 & -r & q \\
r & 0 & -p \\
-q & p & 0
\end{array}\right]_{B}\left[\begin{array}{ccc}
I_{x x} & 0 & I_{x z} \\
0 & I_{y y} & 0 \\
I_{z x} & 0 & I_{z z}
\end{array}\right]_{B}\left[\begin{array}{l}
p \\
q \\
r
\end{array}\right]_{B}=\left[\begin{array}{c}
M_{x} \\
M_{y} \\
M_{z}
\end{array}\right]_{B}
$$

\subsubsection{Attitude Equations}

As illustrated in Figure 14, the orientation of $C S_{B}$ with respect to $C S_{E}$ is determined by the three aircraft Euler angles $\varphi, \theta, \psi$ (roll, pitch, and yaw attitude angles, respectively). The corresponding rotations define the rotation vector of aircraft rigid body $B$ (associated to $C S_{B}$ ) with respect to the Earth (or $C S_{E}$ ):

or:

$$
\begin{gathered}
E \vec{\omega}^{B}=\overrightarrow{\dot{\psi}}+\overrightarrow{\dot{\theta}}+\overrightarrow{\dot{\varphi}} \\
E \vec{\omega}^{B}=\dot{\psi} \vec{v}_{1}+\dot{\theta} \vec{v}_{2}+\dot{\varphi} \vec{v}_{3}
\end{gathered}
$$

where $\vec{v}_{1}, \vec{v}_{2}$, and $\vec{v}_{3}$ are unit vectors along the respective axes of rotation. In components with respect to $C S_{B}$, this equation yields:

$$
\left[\begin{array}{l}
p \\
q \\
r
\end{array}\right]_{B}=\dot{\psi}\left[\vec{v}_{1}\right]_{B}+\dot{\theta}\left[\vec{v}_{2}\right]_{B}+\dot{\varphi}\left[\vec{v}_{3}\right]_{B}
$$


and, finally:

$$
\left[\begin{array}{l}
p \\
q \\
r
\end{array}\right]_{B}=\left[\begin{array}{ccc}
1 & 0 & -\sin (\theta) \\
0 & \cos (\varphi) & \sin (\varphi) \cos (\theta) \\
0 & -\sin (\varphi) & \cos (\varphi) \cos (\theta)
\end{array}\right]\left[\begin{array}{c}
\dot{\varphi} \\
\dot{\theta} \\
\dot{\psi}
\end{array}\right]
$$

Using matrix inversion we obtain the explicit differential scalar equations:

$$
\left[\begin{array}{c}
\dot{\varphi} \\
\dot{\theta} \\
\dot{\psi}
\end{array}\right]=\left[\begin{array}{ccc}
1 & \sin (\varphi) \tan (\theta) & \cos (\varphi) \tan (\theta) \\
0 & \cos (\varphi) & -\sin (\varphi) \\
0 & \sin (\varphi) / \cos (\theta) & \cos (\varphi) / \cos (\theta)
\end{array}\right]\left[\begin{array}{l}
p \\
q \\
r
\end{array}\right]_{B}
$$

\subsubsection{Trajectory Equations}

By definition, the velocity vector of one particle with respect to another is the time derivative of their corresponding position vector. Therefore, the velocity vector of aircraft center of mass $C$ with respect to $R F_{E}$ can be expressed as:

$$
{ }^{E} \vec{V}^{C}=\frac{{ }_{d \vec{r}}^{E C}}{d t}
$$

where the position vector of the center of mass is considered with respect to the origin of $C S_{E}$. In components with respect to $C S_{E}$, the equivalent scalar equations are:

$$
\left[\frac{{ }^{E} d \vec{r}^{E C}}{d t}\right]_{E}=\left[{ }^{E} \vec{V}^{C}\right]_{E}
$$

With usual notations, the position of aircraft center of mass in Earth axes is expressed as:

$$
\left[\vec{r}^{E C}\right]_{E}=\left[\begin{array}{c}
x_{E} \\
y_{E} \\
z_{E}
\end{array}\right]_{E}
$$

Therefore, equation (31) can be written as:

$$
\left[\begin{array}{c}
\dot{x}_{E} \\
\dot{y}_{E} \\
\dot{z}_{E}
\end{array}\right]_{E}=\left[{ }^{E} \vec{V}^{C}\right]_{E} \quad \text { or } \quad\left[\begin{array}{c}
\dot{x}_{E} \\
\dot{y}_{E} \\
\dot{z}_{E}
\end{array}\right]_{E}=L_{E B}\left[{ }^{E} \vec{V}^{C}\right]_{B}
$$

Finally, the trajectory scalar equations are:

$$
\left[\begin{array}{c}
\dot{x}_{E} \\
\dot{y}_{E} \\
\dot{z}_{E}
\end{array}\right]_{E}=\left[\begin{array}{ccc}
\cos (\psi) \cos (\theta) & \cos (\psi) \sin (\theta) \sin (\varphi)-\sin (\psi) \cos (\varphi) & \cos (\psi) \sin (\theta) \cos (\varphi)+\sin (\psi) \sin (\varphi) \\
\sin (\psi) \cos (\theta) & \sin (\psi) \sin (\theta) \sin (\varphi)+\cos (\psi) \cos (\varphi) & \sin (\psi) \sin (\theta) \cos (\varphi)-\cos (\psi) \sin (\varphi) \\
-\sin (\theta) & \cos (\theta) \sin (\varphi) & \cos (\theta) \cos (\varphi)
\end{array}\right]\left[\begin{array}{c}
u \\
v \\
w_{B}
\end{array}\right]_{B}
$$

\subsubsection{Forces and Moments}

The external forces and moments typically consist of, respectively:

$$
\sum_{i} \vec{F}_{i}=\vec{F}_{A}+\vec{F}_{P}+\vec{G}+\vec{F}_{\text {other }}
$$




$$
\sum_{i} \vec{M}_{i}^{C}=\vec{M}_{A}+\vec{M}_{P}+\vec{M}_{\text {other }}
$$

where: $\quad \vec{F}_{A}=$ the resultant of all aerodynamic forces

$$
\begin{aligned}
& \vec{F}_{P} \quad=\text { the resultant of all propulsion forces } \\
& \vec{G} \quad=\text { gravity } \\
& \vec{F}_{\text {other }}=\text { the resultant of other external forces } \\
& \vec{M}_{A} \quad=\text { the resultant of all aerodynamic moments } \\
& \vec{M}_{P} \quad=\text { the resultant of all propulsion moments } \\
& \vec{M}_{\text {other }}=\text { the resultant of other external moments }
\end{aligned}
$$

Aerodynamic and propulsion forces and moments are, in general, functions of the four state vectors and the controls. It should be noted that aerodynamic forces and moments depend directly on the relative velocity of aircraft with respect to the atmosphere ( $\left.{ }^{W} \vec{V}^{C}\right)$. The velocity of the atmosphere with respect to the Earth will be referred to as "wind velocity" and the corresponding vector will be denoted by $\vec{W}$. Therefore:

$$
{ }^{E} \vec{V}^{C}={ }^{W} \vec{V}^{C}+\vec{W}
$$

\subsection{General Expression of Wind Velocity}

A simplified model of atmospheric phenomena can be developed if the main components are summed such that:

$$
\vec{W}=\vec{W}_{c}+\vec{W}_{T}+\vec{W}_{G}+\vec{W}_{S}
$$

where $\vec{W}_{c}$ is the constant wind vector; $\vec{W}_{T}$ is the contribution to wind velocity due to atmospheric turbulence; $\vec{W}_{G}$ represents a wind gust of constant magnitude over a limited period of time; $\vec{W}_{S}$ is capturing the effects of wind shear modeled as spatial gradients.

Within the WVU UAV simulation environment, the components in body axes of total air

velocity $\left([\vec{W}]_{B}\right)$ must be calculated and provided to the modules that calculate the aerodynamic forces and moments:

$$
[\vec{W}]_{B}=\left[\vec{W}_{c}\right]_{B}+\left[\vec{W}_{T}\right]_{B}+\left[\vec{W}_{G}\right]_{B}+\left[\vec{W}_{S}\right]_{B}
$$




\subsection{Constant Wind}

The constant wind velocity vector is defined by its magnitude $W_{c}$ and the orientation angles $\psi_{W}$ and $\theta_{W}$ with respect to $C S_{E}$. These angles and their sign are defined in the same way as the corresponding angles of the aircraft. Therefore, if $\theta_{W}=0^{\circ}$, the wind velocity vector is in the horizontal plane of $C S_{E}$. For $\psi_{W}=0^{\circ}$, the wind blows along the positive direction of Earth axis $X_{E}$. If $C S_{E}$ and $C S_{B}$ coincide, this situation corresponds to a "tail" wind. For $\psi_{W}=90^{\circ}$, the wind blows along the positive direction of Earth axis $Y_{E}$. If $C S_{E}$ and $C S_{B}$ coincide, this situation corresponds to wind blowing from the left of the pilot. For $\psi_{W}=180^{\circ}$, the wind blows along the negative direction of Earth axis $X_{E}$ ("head" wind). For $\psi_{W}=90^{\circ}$, the wind blows along the negative direction of Earth axis $Y_{E}$ (wind from the right). For non-zero values of $\theta_{W}$, the wind velocity vector will have a vertical component: upward for $\theta_{W}>0$ and downward for $\theta_{W}<0$. With these definitions and conventions, note that:

$$
\begin{aligned}
& {\left[\vec{W}_{c}\right]_{W}=\left[\begin{array}{l}
w_{c x} \\
w_{c y} \\
w_{c z}
\end{array}\right]_{W}=\left[\begin{array}{c}
W_{c} \\
0 \\
0
\end{array}\right]_{W}} \\
& {\left[\vec{W}_{c}\right]_{B}=L_{B E}(\varphi, \theta, \psi) L_{E W}\left(\theta_{W}, \psi_{W}\right)\left[\vec{W}_{c}\right]_{W}}
\end{aligned}
$$

The user must specify the constant wind scenario by providing the values for the three defining parameters $W_{c}, \theta_{W}$, and $\psi_{W}$.

\subsection{Turbulence}

Turbulence is modeled as a random variation in magnitude and direction of the translational velocity vector of the air mass as a rigid body. Turbulence is assumed to be isotropic, that is its statistical properties are invariant with respect to rotations of the CS. Therefore, the components of the turbulence velocity vector in body axes $\left[\vec{W}_{T}\right]_{B}$ are calculated directly based on a random process with Gaussian probability distribution, such that they exhibit the following power spectral densities $\Phi$, as dictated by the Dryden turbulence model [60]:

$$
\begin{aligned}
& \Phi_{x w}(\Omega)=\sigma_{x w}^{2} \frac{2 L_{x w}}{\pi} \frac{1}{1+\left(L_{x w} \Omega\right)^{2}} \\
& \Phi_{y w}(\Omega)=\sigma_{y w}^{2} \frac{L_{y w}}{\pi} \frac{1+3\left(L_{y w} \Omega\right)^{2}}{\left[1+\left(L_{y w} \Omega\right)^{2}\right]^{2}}
\end{aligned}
$$




$$
\Phi_{z w}(\Omega)=\sigma_{z w}^{2} \frac{L_{z w}}{\pi} \frac{1}{\left[1+\left(L_{z w} \Omega\right)^{2}\right]^{2}}
$$

where the indices $x w, y w$, and $z w$ represent the axes of $C S_{W}$ and $\Omega$ is the spatial frequency. The standard deviations of the velocity vector components along the three axes are $\sigma_{x w}, \sigma_{y w}$, and $\sigma_{z w}$, respectively. They are a measure of turbulence intensity or severity and depend on altitude $H$ according to the relationship:

$$
\text { For } H<2000 \mathrm{ft} \quad \begin{aligned}
\sigma_{x w}=\sigma_{y w} & =\frac{0.1 w_{20}}{(0.177+0.000823 H)^{0.4}} \\
\sigma_{z w} & =0.1 w_{20}
\end{aligned}
$$

where $w_{20}$ is a reference wind velocity at $20 \mathrm{ft}$ considered to be $10 \mathrm{kts}$ for light turbulence, 25 kts for medium turbulence, and 40 kts for severe turbulence. Thus, the turbulence intensity for low constant wind is $1 \%, 2.5 \%$ and $4 \%$ (low, medium and severe turbulence respectively), and for high speed constant wind case, the turbulence intensity is $0.4 \%, 1 \%$ and $1.6 \%$ (low, medium and severe turbulence respectively).

$$
\text { For } H \geq 2000 \mathrm{ft} \quad \sigma_{x w}=\sigma_{y w}=\sigma_{z w}
$$

The following values are considered: $\sigma=5 \mathrm{ft} / \mathrm{s}$ for light turbulence, $\sigma=10 \mathrm{ft} / \mathrm{s}$ for medium turbulence, and $\sigma=15 \cdots 21 \mathrm{ft} / \mathrm{s}$ for severe turbulence. Therefore, the turbulence intensity for low constant wind is $0.5 \%, 1 \%, 1.5 \%$ and $2.1 \%$ (low, medium and severe turbulence respectively), and for high speed constant wind case, the turbulence intensity is $0.2 \%, 0.4 \%, 0.6 \%-0.84 \%$ (low, medium and severe turbulence respectively).

$L_{x w}, L_{y w}$, and $L_{z w}$ represent scaling lengths for power spectra. For lower altitudes, they are calculated with the following relationships:

$$
L_{x w}=L_{y w}=\frac{H}{(0.177+0.000823 H)^{1.2}}, \quad L_{z w}=H
$$

For altitudes above $2000 \mathrm{ft}$ :

$$
L_{x w}=L_{y w}=L_{z w}=1750 \mathrm{ft}
$$

The numerical implementation of the Dryden turbulence model relies on passing white noise signals through low order linear filters such that the outputs of the filters, random functions, exhibit the power spectral densities of the Dryden model in equations (xyz). White noise is defined as a signal whose correlation function is an impulse and whose power spectral density function is constant. 
Let the power spectral density of a white noise signal be denoted as $\Phi_{w n}$. If the transfer function of a linear filter is $F(s)$, the output of the filter in response to a random input will be a random function $f$. The power spectral density of $f$ will be given by:

$$
\Phi_{\mathrm{f}}(\Omega)=\Phi_{w n}|F(j \Omega)|^{2}
$$

The following linear filters must be used on each axis, respectively, in order for the outputs to have the spectra described by equations (xyz):

$$
\begin{aligned}
& F_{x w}(s)=\frac{1}{1+L_{x w} s} \\
& F_{y w}(s)=\frac{1+\sqrt{3} L_{y w} s}{\left(1+L_{z w} s\right)^{2}} \\
& F_{z w}(s)=\frac{1+\sqrt{3} L_{z w} s}{\left(1+L_{z w} s\right)^{2}}
\end{aligned}
$$

Note that the transfer function is obtained with a Laplace transform involving spatial frequency. The conversion to time domain can be done considering that:

$$
\Omega=\left(\mathrm{V}_{\mathrm{r}} \mathrm{t}\right)^{-1}
$$

where $V_{r}$ is a reference velocity of the vehicle. The inputs to the filters are random un-correlated numbers with Gaussian distribution. Their standard deviations along the three axes can be determined with the following relationships, where $\Delta \Omega$ is the spatial integration step.

$$
\begin{gathered}
\sigma_{w n}=\sigma_{x w} \sqrt{\frac{2 L_{x w}}{\Delta \Omega}} \\
\sigma_{w n}=\sigma_{y w} \sqrt{\frac{2 L_{y w}}{\Delta \Omega}} \\
\sigma_{w n}=\sigma_{z w} \sqrt{\frac{2 L_{z w}}{\Delta \Omega}}
\end{gathered}
$$

The user must specify the level of turbulence as "none", "low", "medium", or "severe".

\subsection{Wind Gust}

The wind gust is defined by its magnitude $W_{G}$, the orientation angles $\theta_{G}$ and $\psi_{G}$ with respect to $C S_{E}$, the duration of the gust $D_{G}$, and the location of the gust expressed implicitly in terms of simulation time $T_{G}$. This formulation was found to be more convenient for simulation and analysis purposes. The gust orientation angles and their sign are defined in the same way as 
the corresponding angles of the constant wind. With these definitions and conventions, note that:

$$
\begin{aligned}
& {\left[\vec{W}_{G}\right]_{G}= \begin{cases}{\left[\begin{array}{lll}
W_{G} & 0 & 0
\end{array}\right]_{G}^{T}} & \text { if } T_{G} \leq \text { simulation time }<T_{G}+D_{G} \\
{\left[\begin{array}{lll}
0 & 0 & 0
\end{array}\right]_{G}^{T}} & \text { otherwise }\end{cases} } \\
& {\left[\vec{W}_{G}\right]_{B}=L_{B G}\left[\vec{W}_{G}\right]_{G}=L_{B E}(\varphi, \theta, \psi) L_{E G}\left(\theta_{G}, \psi_{G}\right)\left[\vec{W}_{G}\right]_{G}}
\end{aligned}
$$

The user must specify the wind gust scenario by providing the values for the five defining parameters $W_{G}, \theta_{G}, \psi_{G}, D_{G}$, and $T_{G}$.

\subsection{Wind Shear}

Wind shear is a complex atmospheric phenomenon consisting of abrupt variations in wind velocity over relatively reduced distances. These variations are produced by non-zero spatial wind gradients that exist in a certain limited region of the physical space denoted as $R_{W S}$. The non-zero spatial wind gradients can be modeled as a wind shear tensor $W_{S}$ whose components will represent the derivatives of the three wind velocity vector projections with respect to distance along the three axes [44, 46]. Therefore:

$$
\underline{W_{s}}=\frac{W_{d \vec{W}}}{\underline{d \vec{r} S C}}= \begin{cases}0 & \text { if } C \notin R_{W S} \\ \neq 0 & \text { if } C \in R_{W S}\end{cases}
$$

In components with respect to $C S_{W}$, this equation is equivalent to:

$$
\left[\underline{W_{s}}\right]_{W}=\left[d w_{i j}\right]_{W}=\left[\begin{array}{lll}
\frac{d w_{c x}}{d X_{W}} & \frac{d w_{c x}}{d Y_{W}} & \frac{d w_{c x}}{d Z_{W}} \\
\frac{d w_{c y}}{d X_{W}} & \frac{d w_{c y}}{d Y_{W}} & \frac{d w_{c y}}{d Z_{W}} \\
\frac{d w_{c z}}{d X_{W}} & \frac{d w_{c z}}{d Y_{W}} & \frac{d w_{c z}}{d Z_{W}}
\end{array}\right]_{W}
$$

Note that:

$$
\underline{W_{s}}= \begin{cases}0 & \text { if } d w_{i j}=0, \quad \forall i, j \\ \neq 0 & \text { otherwise }\end{cases}
$$

Wind shear may be modeled by a vector $\vec{W}_{S}$ that is the dot-product between the spatial gradient tensor $\left(\frac{W_{d \vec{W}}}{d \vec{r} S C}\right)$ and the position vector of aircraft center of mass with respect to a fixed point in $C S_{S}\left(\vec{r}^{S C}\right)$ :

$$
\vec{W}_{S}=\frac{W_{d \vec{W}}}{\underline{d \vec{r} S C}} \cdot \vec{r}^{S C}
$$


Note that this relationship holds for as long as the aircraft is inside the atmospheric volume in which the phenomenon occurs. It should be noted that, once the aircraft exits $R_{W S}$ at $t=t_{\text {exit }}$, the gradient tensor becomes 0 ; however, the constant wind value is altered by the effects of the non-zero gradient accumulated while aircraft was inside $R_{W S}$. Therefore, for simulation purposes, the accurate definition of $\vec{W}_{S}$ is:

$$
\vec{W}_{S}(t)= \begin{cases}\frac{W_{d \vec{W}}}{d \vec{r} S C} \cdot \vec{r} S C & \text { for } t<t_{\text {exit }} \\ \vec{W}_{S}\left(t_{\text {exit }}\right) & \text { for } t \geq t_{\text {exit }}\end{cases}
$$

In most applications for UAV design and performance analysis, the absolute spatial location of $R_{W S}$ is not so important. More important is rather the relative location of $R_{W S}$ with respect to aircraft trajectory. This facilitates the analysis of what happens when the aircraft experiences wind shear while in straight level flight, turn, climb, or any other maneuver. Therefore, it is more convenient to assimilate the location of $R_{W S}$ within the physical space to a specified time during simulation $T_{W S}$. The extent of the wind shear affected region $R_{W S}$ can then be assimilated to a total duration $D_{W S}$. With these assumptions, the wind gradient tensor can be expressed as:

$$
\frac{w_{d \vec{W}}}{\underline{d \vec{r} S C}}= \begin{cases}\neq 0 & \text { for } T_{W S} \leq t \leq T_{W S}+D_{W S} \\ 0 & \text { otherwise }\end{cases}
$$

and the effect of the wind shear on the total atmospheric velocity vector is:

$$
\vec{W}_{S}(t)= \begin{cases}\frac{W_{d \vec{W}}}{d \vec{r} S C} \vec{r}^{S C} & \text { for } t<T_{W S}+D_{W S} \\ \vec{W}_{S}\left(t=T_{W S}+D_{W S}\right) & \text { for } t \geq T_{W S}+D_{W S}\end{cases}
$$

The components of $\vec{W}_{S}$ with respect to body axes, which are necessary for calculation of aerodynamic forces and moments, are determined as follows:

$$
\left[\vec{W}_{S}\right]_{B}=L_{B E}\left[\vec{W}_{S}\right]_{E}=L_{B E} L_{E W}\left[\vec{W}_{S}\right]_{W}=L_{B E} L_{E W}\left[\vec{W}_{S}\right]_{W}
$$

Therefore:

$$
\left[\vec{W}_{S}\right]_{B}= \begin{cases}L_{B E} L_{E W}\left[\frac{{ }^{W} \vec{w}}{d \vec{r} S C}\right]_{W}\left[\vec{r}^{S C}\right]_{W} & \text { for } t<T_{W S}+D_{W S} \\ L_{B E} L_{E W}\left[\vec{W}_{S}\left(T_{W S}+D_{W S}\right)\right]_{W} & \text { for } t \geq T_{W S}+D_{W S}\end{cases}
$$

To simplify notation, let us focus only on the $t<T_{W S}+D_{W S}$ case. 


$$
\left[\vec{W}_{S}\right]_{B}=L_{B E} L_{E W}\left[\begin{array}{lll}
\frac{d w_{c x}}{d X_{W}} & \frac{d w_{c x}}{d Y_{W}} & \frac{d w_{c x}}{d Z_{W}} \\
\frac{d w_{c y}}{d X_{W}} & \frac{d w_{c y}}{d Y_{W}} & \frac{d w_{c y}}{d Z_{W}} \\
\frac{d w_{c z}}{d X_{W}} & \frac{d w_{c z}}{d Y_{W}} & \frac{d w_{c z}}{d Z_{W}}
\end{array}\right]_{W} \quad L_{W E}\left[\vec{r}^{S C}\right]_{E}
$$

Note that:

$$
\begin{aligned}
& \vec{r}^{S C}=\vec{r}^{E C}-\vec{r}^{E S} \\
& \vec{r}^{E S}=\vec{r}^{E C}\left(t=T_{W S}\right)
\end{aligned}
$$

In components with respect to Earth axes, we obtain:

$$
\begin{gathered}
{\left[\vec{r}^{S C}\right]_{E}=\left[\vec{r}^{E C}\right]_{E}-\left[\vec{r}^{E S}\right]_{E}} \\
{\left[\vec{r}^{S C}\right]_{E}=\left[\begin{array}{l}
x_{E} \\
y_{E} \\
z_{E}
\end{array}\right]_{E}-\left[\begin{array}{l}
x_{E}\left(t=T_{W S}\right) \\
y_{E}\left(t=T_{W S}\right) \\
z_{E}\left(t=T_{W S}\right)
\end{array}\right]_{E}}
\end{gathered}
$$

Then:

$$
\left[\vec{W}_{S}\right]_{B}=L_{B E} L_{E W}\left[\begin{array}{lll}
\frac{d w_{c x}}{d X_{W}} & \frac{d w_{c x}}{d Y_{W}} & \frac{d w_{c x}}{d Z_{W}} \\
\frac{d w_{c y}}{d X_{W}} & \frac{d w_{c y}}{d Y_{W}} & \frac{d w_{c y}}{d Z_{W}} \\
\frac{d w_{c z}}{d X_{W}} & \frac{d w_{c z}}{d Y_{W}} & \frac{d w_{c z}}{d Z_{W}}
\end{array}\right]_{W} L_{W E}\left[\begin{array}{l}
x_{E}-x_{E}\left(t=T_{W S}\right) \\
y_{E}-y_{E}\left(t=T_{W S}\right) \\
z_{E}-z_{E}\left(t=T_{W S}\right)
\end{array}\right]_{E}
$$

The user must first define the constant wind scenario and then provide the 9 components of the wind gradient tensor with respect to wind axes, the simulation time $T_{W S}$ when the wind spatial gradients become active (equivalent to the location of the wind shear phenomenon), and the duration $D_{W S}$ of non-zero gradients (equivalent to exiting the region of the wind shear phenomenon). 


\section{SIMULATION ENVIRONMENT}

To simulate autonomous flight and fault-tolerant control laws, a simulation environment is required that allows for changes in parameters and visual cues of the aircraft simulated. In this chapter, the WVU simulation environment used in this research will be discussed, along with the metrics for evaluating the UAV performance under different atmospheric scenarios.

\subsection{WVU Simulation Environment}

This research effort was only possible due to the WVU UAV Simulation Environment that allows for maximum flexibility in terms of trajectory generation and tracking algorithms, aircraft subsystem abnormal conditions, and integration of atmospheric phenomena models [4-6 and 61]. This testing environment was created with the purpose of simulating different fault tolerant scenarios to analyze the performance of tracking controllers. The simulation environment was built in for Matlab/Simulink platform and the visuals of the vehicle and respective environment are provided by FlightGear (open source software) [62 and 63]. In addition, the UAVDashboard interface, integrates the map customized by the user with visuals in a feedback program [4 and 6]. Some important features of the simulation environment are the possibility of looking at parameters values in real time and save or load trajectories created. These feature become crucial when the same trajectory is needed multiple times during a study. Besides autonomous flight, it is also possible to perform manual or formation flight depending on the objective to be studied. A hardware joystick is interfaced with the model along with a throttle to provide the user with manual control. It is possible to interchange parameters and features during the simulation, such as the controllers or the path planners. The user may see plots of the trajectories generated and analyze performance indices of the controllers. The images below (Figure 15, Figure 17 and Figure 18) show the aircraft model, FlightGear and UAVDashboard, respectively. Moreover, the WVU UAV Simulation Environment provided user-friendly capability to integrate the atmospheric phenomena models developed and described in this dissertation. 


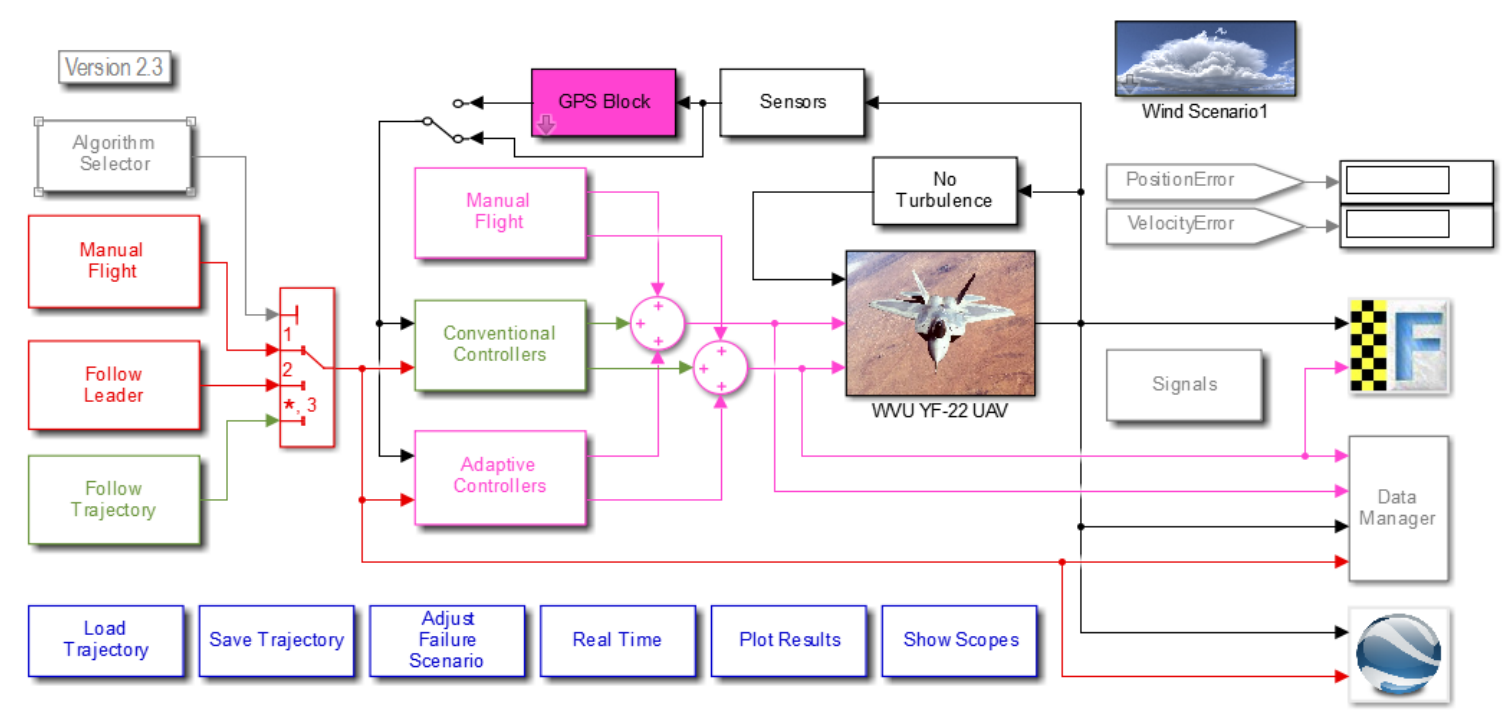

Figure 15- YF22 Aircraft Model.

\subsection{Graphical User Interface (GUI)}

Whenever doing simulations and experiments in any software, it is important to have a user friendly interface that permits the user to vary parameters and variables easily and in a timely manner. Thus, a GUI was developed to facilitate the changes in inputs before the simulation starts, it allows the user to choose among different aircraft models, trajectory tracking algorithms (conventional or adaptive), path planners and failures [5 and 6]. Furthermore, if any variation in the simulation is needed after the GUI is closed, the model is built with a configurable subsystem allowing the user to simply interchange any of the options that were available in the GUI. This interface is shown in Figure 16.

The visuals are presented by FlightGear. It is synchronized with Dashboard and provides 3D motion and visualization of the environment in which the aircraft is exposed. In this project, the visual is of the San Francisco (CA) Bay area. Although this software is used mainly for visual cues without any impact on the experiment, it is synchronized and updates according to the model being used in the simulation environment [4, 6 and 62]. Thus, it permits the user to see changes in the dynamics of the aircraft in real time. The aircraft will yaw, roll, pitch or shake depending on external forces. This feature is very useful because it helps the user to understand what is happening just by watching the aircraft dynamic responses to the inputs. An example of a typical view of FlightGear is shown in Figure 17. 


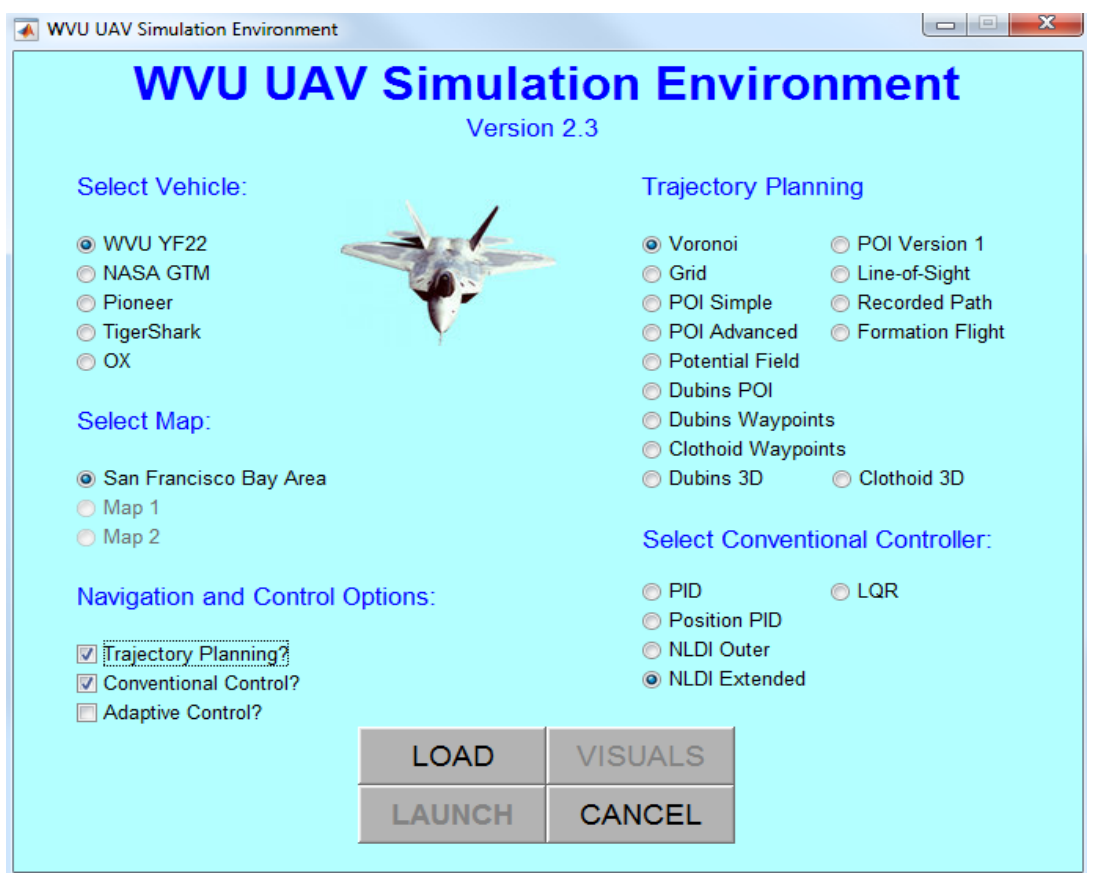

Figure 16- Graphical User Interface (GUI).

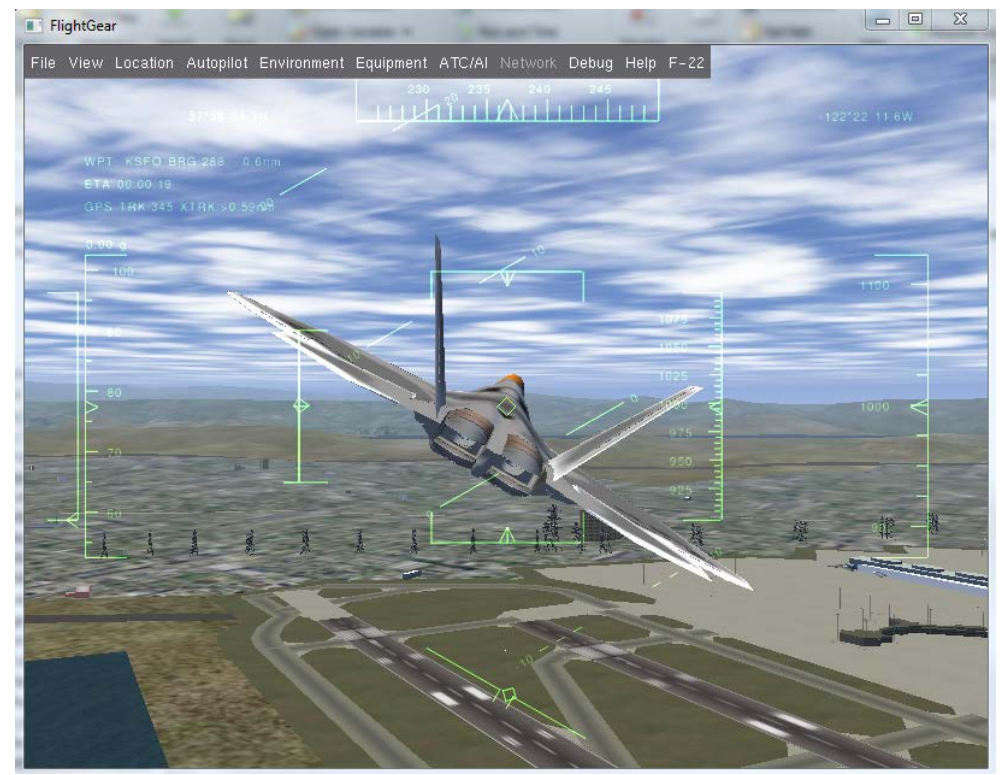

Figure 17- FlightGear Interface.

Another relevant tool utilized for visual information, and is synchronized with the model and FlightGear, is the UAVDashboard. On this interface, it is possible to create scenarios and trajectories by adding threat zones, obstacles, points of interest, waypoints or load pre-recorded paths for repeatability. It shows on a 2D perspective the trajectory planned, plotted against the actual path that the aircraft is following for conveniently and easy perception of tracking errors. 
The Dashboard is built in the Microsoft Visual C\# programming language and presents the same region or location shown in FlightGear connected via UDP [6]. To initialize any simulation, the aircraft and its starting coordinate point have to be placed into the environment of Dashboard. Following this process, addition of risk zones is needed or for pre-recorded path could be loaded instead. An example of the expected view of the UAVDashboard interface can be seen in Figure 18.

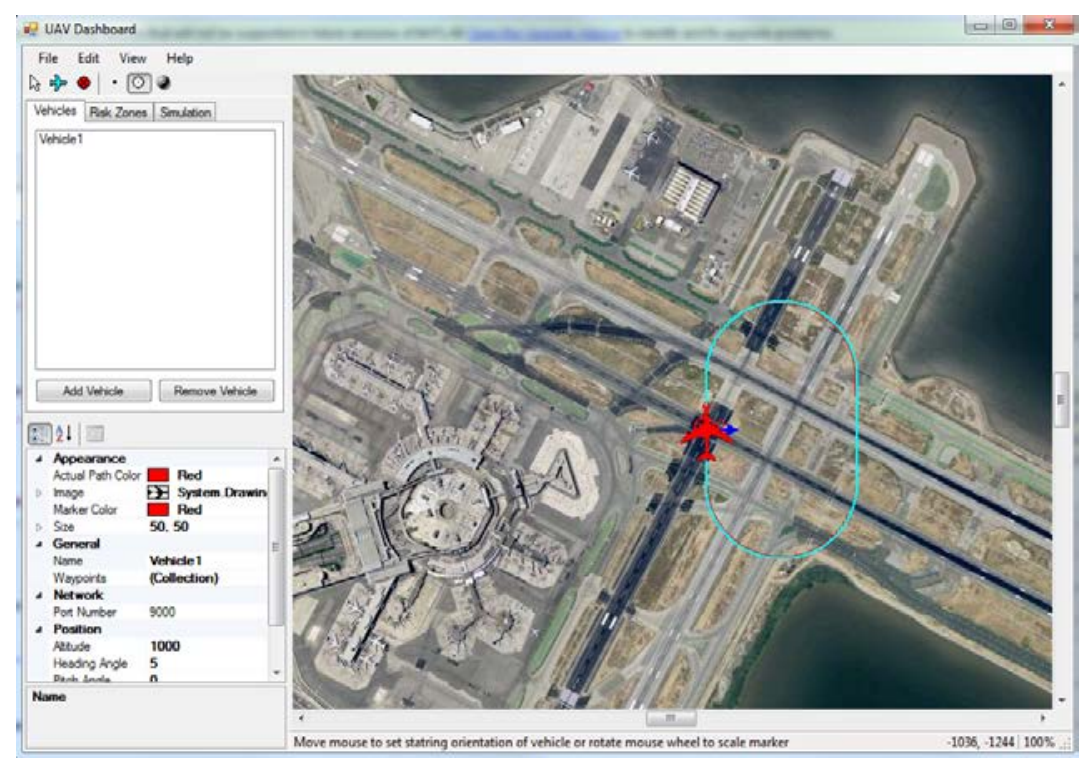

Figure 18- Dashboard Interface.

\subsection{Aircraft Models}

There are five different aircraft models that can be chosen in the GUI, they are the WVU YF-22, NASA GTM, Pioneer drone, TigerShark and OX. Regarding the last three UAV military aircraft mentioned, a thorough information of the implementation into the simulation environment and modeling can be found in Karas [6]. For the NASA Generic Transport Model aircraft, it is a $5.5 \%$ scale of the Boeing 757 commercial jet and contains significant abnormal conditions simulation. Despite the various models available, the fault-tolerant wind model implemented during this research only deals with the WVU YF-22, which is an UAV mimicking the Lockheed/Boeing fighter aircraft used by the U.S Airforce. In fact, this model is the most complete and trustworthy among the others, it contains more options for fault-tolerant experiments and analysis, and is the only one validated, hence, it was chosen for this research effort. The WVU YF$22 \mathrm{UAV}$ is a research aircraft developed at WVU and is approximately $15 \%$ of the actual size aircraft and can do missions of about 12 minutes of flight duration. Its wingspan is $6^{\prime} 6^{\prime \prime}$ and the 
UAV is able to carry up to $12 \mathrm{lbs}$ of payload with maximum takeoff weight of $50 \mathrm{lbs}$ [6 and 64]. Figure 19 shows the WVU UAVs research aircraft.

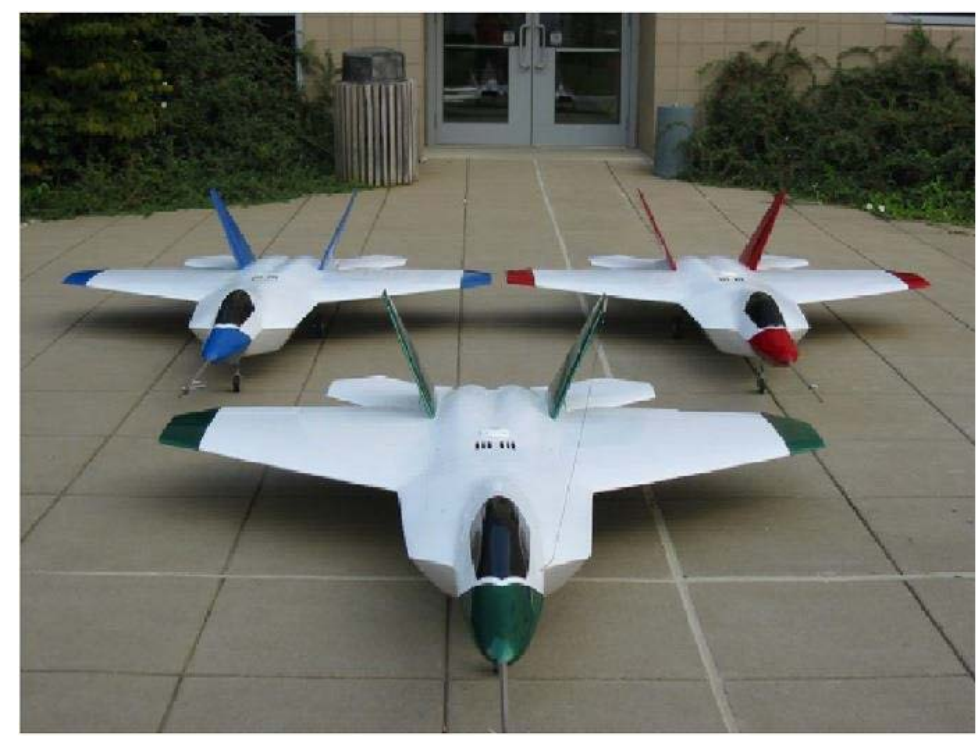

Figure 19- Research UAV YF-22 [4].

More information on this research aircraft can be found in [64] where detailed aerodynamics and dynamics of the aircraft are meticulously described. Numerous flights were done using this UAV, and the flight data recorded was used for several research projects, including parameter identification techniques, where the WVU YF-22 fault-tolerant simulation model block was created. During test flights, variables such as aerodynamic coefficients $\mathrm{Cd}, \mathrm{Cz}$ and $\mathrm{Cl}$, were saved and used to construct differential equations to build the non-linear model WVU YF-22. Additionally, a linear model was also developed with the purpose of developing trajectory tracking algorithms. A thorough discussion of such approach can be found in [4].

\subsection{Abnormal Conditions Simulation Models}

Within the simulation environment a few abnormal conditions models have been developed and implemented with the goal of testing the robustness of fault tolerant tracking controllers under such upset scenarios. Several alternatives are possible to be tested for actuator or sensor failures, along with Global Positioning System (GPS) errors or atmospheric influences.

For the actuator failures, two types are available, jammed/locked or damaged control surfaces for rudder, stabilators and ailerons. Once the control surface is locked in a pre-defined position, it will stay there for the remaining time of the simulation. Therefore, the differences in 
moments and aerodynamic forces between the surface pair causes the aircraft to change its dynamics. More about the subject can be found in [65].

Sensors are a very important source of measurements that are used by the aircraft computer to help maintain stability during flight. Although there are a wide range of sensors in an aircraft, the gyros are among the most essential to flight. Thus, failures in the three channels can be simulated for the gyro with different levels of severity. A bias is added to the sensor and that changes the output value, which results in an alteration of the dynamic behavior of the aircraft.

GPS are also susceptible to abnormal operations and can significantly influence the trajectory of an aircraft. Thus, attempting to better understand the effects of such failures, AlSinbol [5] developed a GPS model block and GUI. There, it is possible to create abnormalities in the GPS in update rate, pure time delay and measurement errors in Earth axis of the center of mass position and velocity of the aircraft.

Turbulence is classified in the WVU simulation environment according to its severity as: no turbulence, low turbulence, moderate turbulence and high turbulence. Following the main objective of this research, a wind model block and GUI (Figure 20) was added into the turbulence block and allows for simulation of constant wind, winds gust and wind shear. With respect to the constant wind, magnitude, heading angle and pitch angle have to be inputted in order to initiate the simulation. These directions are corresponding to the starting point and direction of the aircraft input on Dashboard. For wind gust simulation, the duration and time of occurrence are important factors that need to be defined. Moreover, the user is required to specify the direction of the wind and its magnitude.

Lastly, a wind shear model was implemented. In order to be simulated, the constant wind tab needs to be completed. The wind shear are gradient components that will increase the current constant wind by a certain amount over distance. Hence, the time of occurrence of the wind shear and its duration are import factors to be settled, as well as the magnitude and direction of the gradient components within the gradient matrix. 


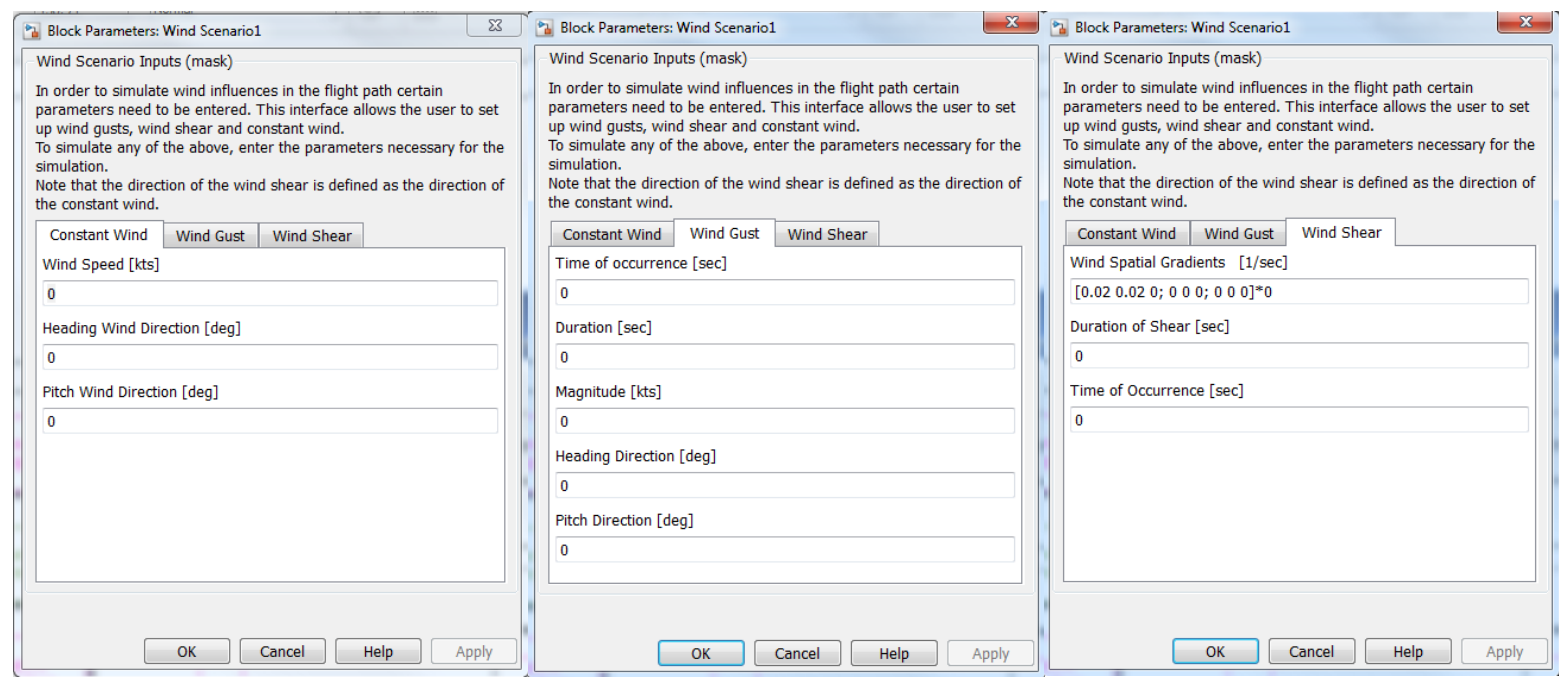

Figure 20- GUls for Constant Wind, Wind Gust and Wind Shear Models.

\subsection{Trajectory Planning Algorithms}

In this particular project, for the sake of repeatability and to be sure of exposing the aircraft to all directions of wind independent of its starting point, pre-recorded paths were used. A 2D figure 8 with $2 x$ minimum turning radius (Figure 21), an oval geometrical figure with $2 x$ minimum turning radius also in 2D (Figure 22) and a path with 3 Dimensional "s" turns (Figure 23 and Figure 24).

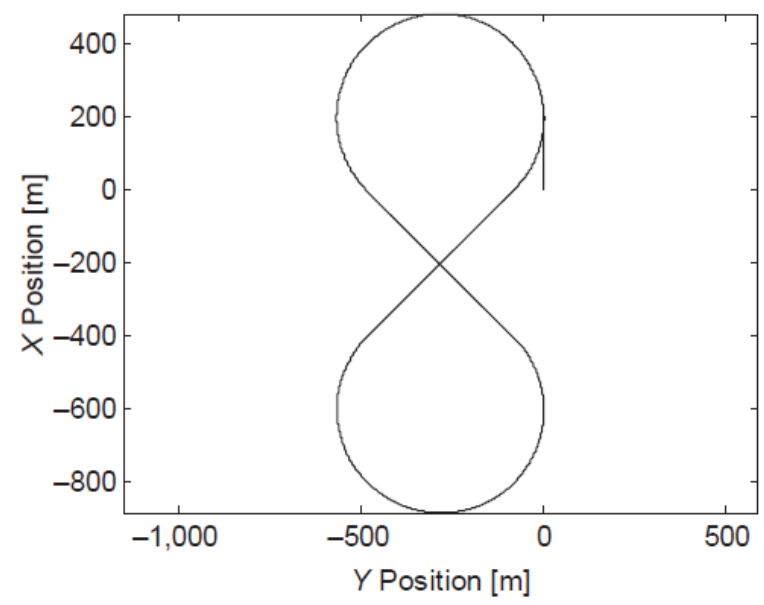

Figure 21- Figure 8 Geometric Path [7]. 


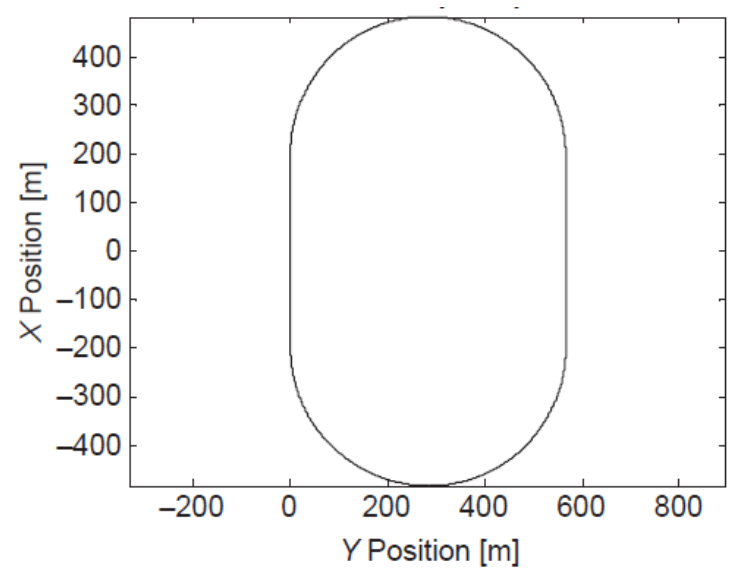

Figure 22- Oval Figure Geometric Path [7].

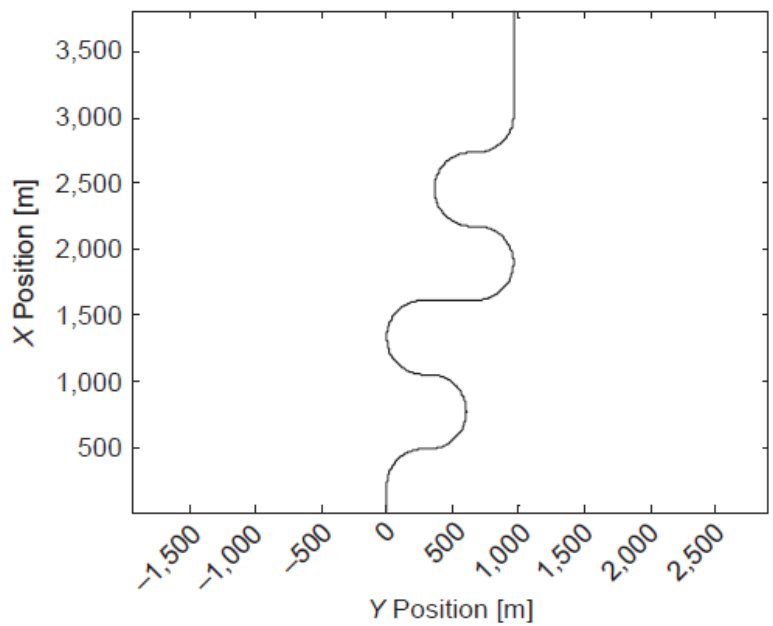

Figure 23- 3D S Turns [7].

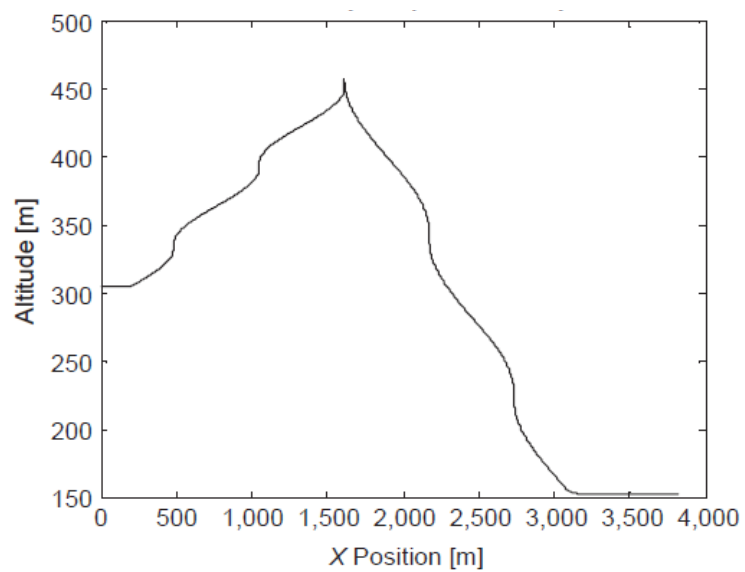

Figure 24- 3D S Turns Vertical Perspective [7]. 
In addition to the three paths above, a path planning algorithm was used to create two other paths to meet the same goal mentioned previously, the Dubins and clothoid path planners in 2 Dimensions. These are pose-based methods that uses waypoints specified by the human operator with poses (headings) to plan the trajectory. According to Wilburn [61], Dubins algorithm is the most common pose-based method for path planning, and for 2D plane, it generates a flyable trajectory by connecting tangent points generated from combinations of circular arcs and straight line segments.

The 2D Clothoid path planner algorithm is less common although is more specialized. This method works by having a continuous curvature profile for the curves created. This approach characterizes a ramp or graduation for the acceleration command input instead of the step input generated by the Dubins. Both methods were created for specific applications. It is necessary to define the start and final pose of the aircraft, then generate the path in between them, therefore some optimization might be needed. The pose is oriented by the coordinates of the center of mass of the aircraft and the tangent to the trajectory. Thus, the Dubins and Clothoid algorithms were developed to solve this optimization problem [6]. An example of the two types of trajectory generation can be seen in the figure below. For more information and details on either path planner algorithm, please refer to [61].

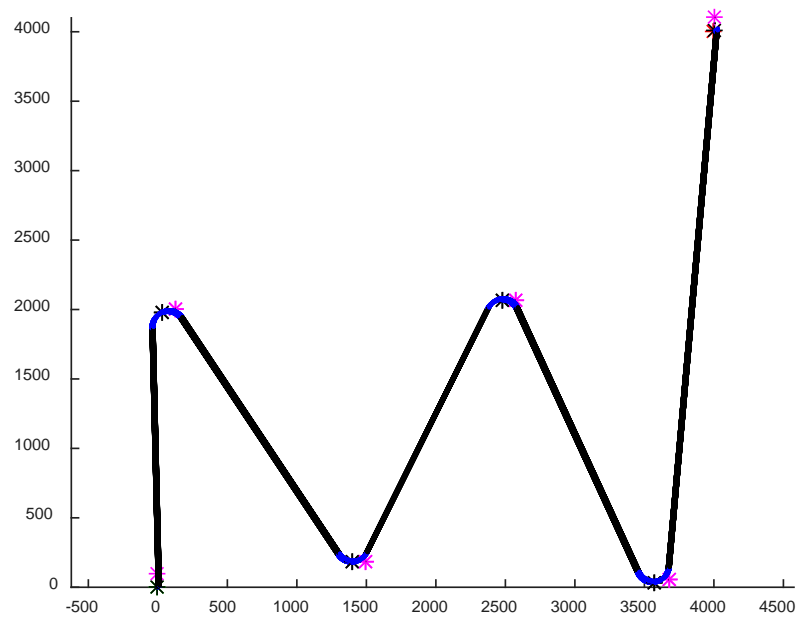

Figure 25- Dubins Path Generation. 


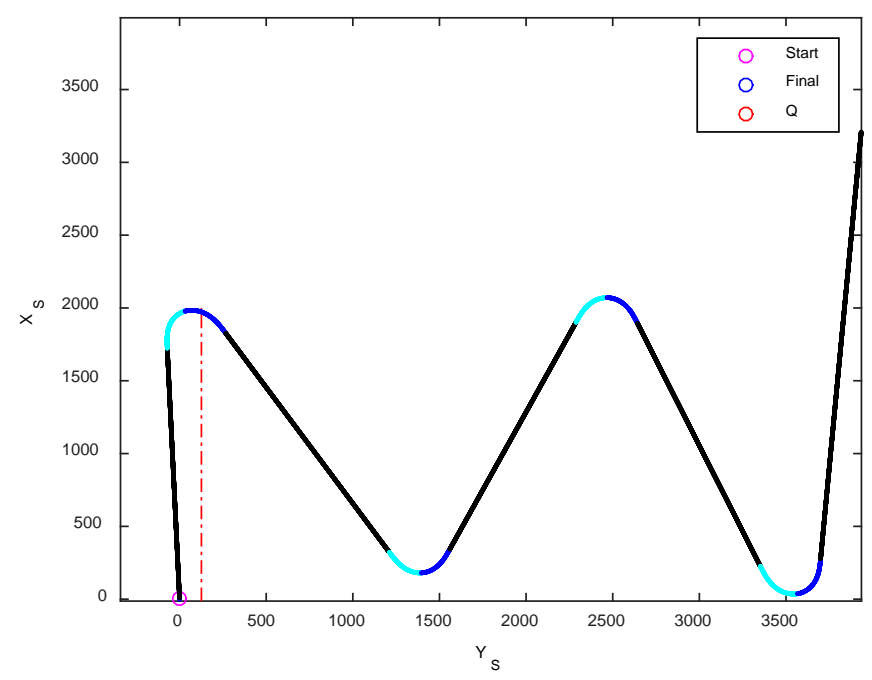

Figure 26- Clothoid Generation.

\subsection{Trajectory Tracking Controllers}

Within the simulation environment, there are two major classes of trajectory tracking controllers that were implemented: fixed parameter control law and adaptive control laws. Both methods will be discussed here since they were used to test the sensitivity of the controllers.

\subsection{1. $\quad$ Fixed-Parameter Control Laws}

There are different approaches for fixed-parameter control laws available in the simulation environment, although, in this study the only one used was the position, proportional, integral and derivative (PPID) compensation. The main purpose of the PPID controller is to minimize errors in the vertical, longitudinal and lateral position [6 and 7]. It compares the desired position and velocity from the commanded trajectory with readings of the aircraft GPS sensor. The difference is the error. The design of the PID controllers is based on lateral and longitudinal control, each containing an inner and outer loop [5]. The ailerons, elevators and throttle are used in the inner loop to control bank and pitch angles while the velocity, lateral, longitudinal and vertical distances are controlled in the outer loop. The errors in position and velocity are computed in terms of the body axis and passed on to the outer loop controller. Moreover, the PID controller will compute the necessary bank angle, pitch angle and throttle needed to correct the lateral, longitudinal and vertical errors. Finally, the inner loop gives the necessary command to the ailerons, throttle and elevator to achieve the required values to compensate the errors [5]. This controller provides good tracking control, although in presence of upset conditions it may lack robustness [4 and 6]. A schematic of PPID controller can be seen in Figure 27 and the schematic works for adaptive parameter as well. 


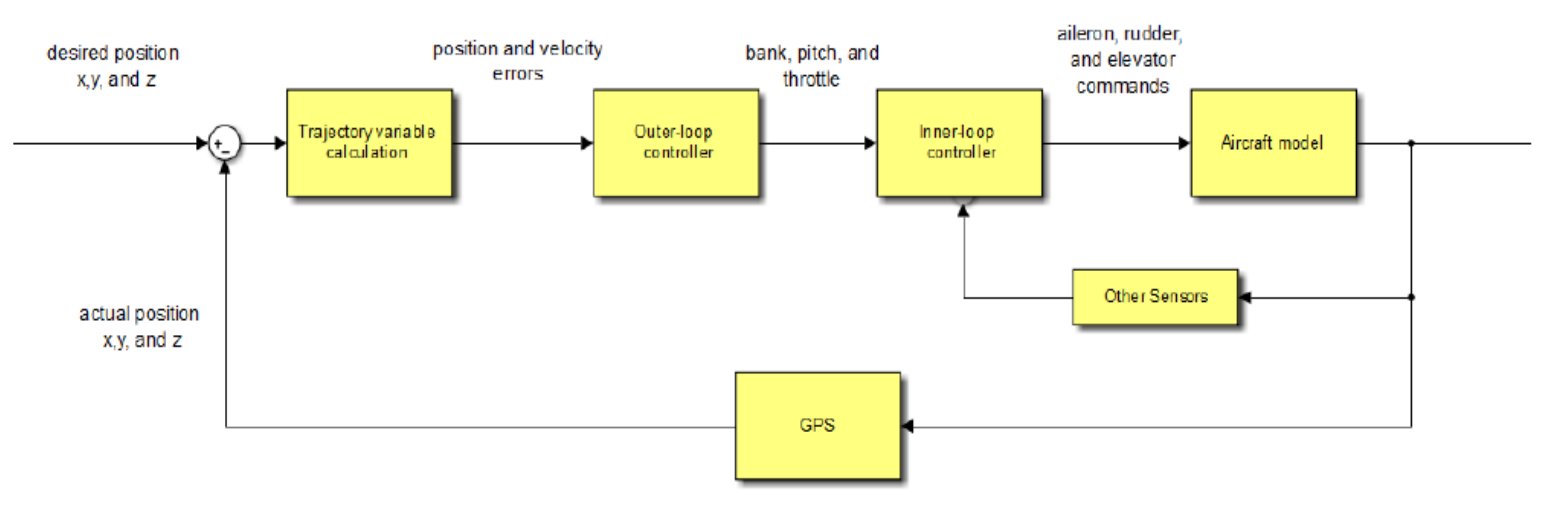

Figure 27- Schematic of PID Controller [5].

\subsubsection{Adaptive Control Laws}

While conventional controllers are more robust, it usually do not perform well under abnormal conditions. This is due to fact that conventional controllers are more sensitive to uncertainties [4]. Therefore, adaptive controllers were developed to improve this issue, they are built to be robust and have good performance under imprecision or abnormal conditions. Adaptive controllers, unlike conventional controllers, have gains that are adaptable during flight in real-time to compensate for changes in flight conditions or subsystems. According to Al-Sinbol [5], an adaptive mechanism is added to the fixed parameter architecture (therefore structure is the same) to modify gains. This is expected to improve performance under conditions that depart from the ones assumed when the fixed parameter control laws were designed, such as excessive atmospheric phenomena or subsystem failure. For more details on adaptive control, please refer to $[4,66$ and 67].

\subsubsection{NLDI Extended}

The NLDI extended (nonlinear dynamic inversion) controller is a NLDI-based control used in the inner-loop and outer-loop. "The inner-loop controller is divided into two sub-controllers referred to as slow mode and fast mode" according to Wilburn [4]. In the slow mode, the roll, pitch and yaw commands are calculated. Then, the fast mode uses the commands provided by the slow mode to produce control surface commands for ailerons, elevators and rudders. For more information on NLDI Extended controller, please refer to [4]. 


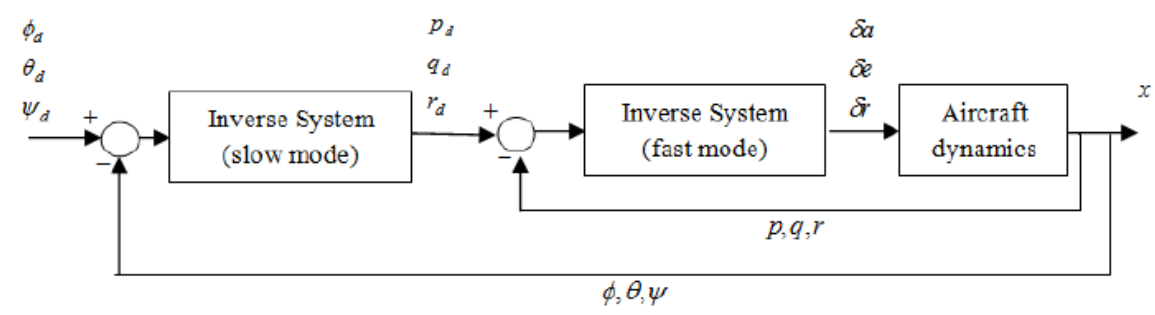

Figure 28-Schematic of NLDI Extended Controller.

\subsection{Performance Metrics}

In order to know if a controller has good or poor performance, a set of performance metrics were developed within the simulation environment and they are based on two criteria [67]. The first objective is that the aircraft should follow the commanded trajectory with the least possible tracking errors, thus, it is based on the actual accuracy of the controller. In calculating the error, the parameters measured are the maximum, average and standard deviation of the absolute error [4 and 5]. In order to measure this, the errors are defined in the horizontal XY plane, along the $Z$ axis and combined in the 3-dimensional space. The error in the XY plane can be defined as in equation below.

$$
e_{x y}=\sqrt{\left[x_{c}(t)-x(t)\right]^{2}+\left[y_{c}(t)-y(t)\right]^{2}}
$$

Where $t$ represents time, $X$ and $Y$ are the commanded position of the aircraft. The actual position of the aircraft is denoted by subscript $c$. The error in $Z$ is calculated similarly, with $Z_{c}$ being the actual versus $Z$ the commanded trajectory, the equation is then define as:

$$
e_{z}=\left|z_{c}(t)-z(t)\right|
$$

The 3D space tracking errors for XYZ can be calculated as:

$$
e_{x y z}(t)=\sqrt{\left[x_{c}(t)-x(t)\right]^{2}+\left[y_{c}(t)-y(t)\right]^{2}+\left[z_{c}(t)-z(t)\right]^{2}}
$$

Based on the equations define above, the trajectory tracking metrics can be calculated for the nine possible indices (average, maximum and standard deviation for $X Y, Z$ and $X Y Z$ (3D)) using the following equations:

Maximum tracking error for XY plane:

$$
e x y_{\max }=\max \left(\left|e_{x y}(t)\right|\right)
$$

Average tracking error for $\mathrm{XY}$ plane:

$$
\operatorname{exy}_{\text {ave }}=\operatorname{mean}\left(\left|e_{x y}(t)\right|\right)
$$


Standard deviation for XY plane:

$$
e x y_{S T D}=S T D\left(\left|e_{x y}(t)\right|\right)
$$

With the equations defined above for trajectory tracking metrics, a specific performance vector can be define as:

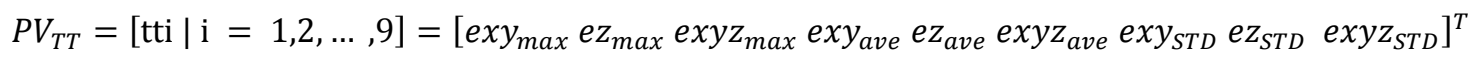

The second objective is related to control activity, this means how much the controller is working to be able to maintain the aircraft in the commanded trajectory, with minimum possible deflection of the control surfaces, without reaching saturation. Commands are generated by trajectory controller with the constraint that saturation is to be avoided. The deflections should be gradual in a way that the aircraft will be able to obey the command from the controller. Hence, the measurement of the actuator signals (control activity) can be done by integrating the absolute value of the rate of change of the signal given, and the saturation can be calculated as a percentage of the time steps in which the surface is operating at its maximum extent [5]. Let the deflection of the surface control elevator be $\boldsymbol{\delta} \boldsymbol{e}$, then the indices of control activity is defined by the equations:

Integration of the stabilator rate of change:

$$
I \dot{\delta}_{x}=\frac{1}{T} \int_{0}^{T}\left|\dot{\delta}_{x}(t)\right| d t
$$

Stabilator saturation index:

$$
S_{\delta_{x}}=\frac{100}{T} \int_{0}^{T} \tilde{\delta}_{x}(t) d t
$$

Where:

$$
\tilde{\delta}_{x}(t)=\left\{\begin{array}{l}
0, \text { if } \delta_{x}>\delta_{x_{\min }} \wedge \delta_{x}<\delta_{x_{\max }} \\
1, \text { if } \delta_{x} \leq \delta_{x_{\min }} \vee \delta_{x} \geq \delta_{x_{\max }}
\end{array}\right.
$$

These same equations can be used to calculate for aileron (a), rudder ( $r$ ) and throttle $(t)$ as well, where the deflection of the elevator would be substituted by any of the other surfaces deflections mentioned, and throttle would be related to displacement. Using the definitions above, another specific vector can be defined for control activity as:

$$
P V_{C A}=[\text { cai } \mid \mathrm{i}=1,2, \ldots, 8]=\left[\begin{array}{lllll}
I \dot{\delta}_{e} & I \dot{\delta}_{a} I \dot{\delta}_{r} I \dot{\delta}_{t} S_{\delta_{e}} S_{\delta_{a}} S_{\delta_{r}} S_{\delta_{t}}
\end{array}\right]^{T}
$$


In combining the accuracy with the activity performance of the controller, another performance metric can be calculated and is here referred to as Total Performance Index (PI). This is done to facilitate the calculations of the various indices (17 total) for one or more controller. The trajectory tracking accuracy can be define as:

$$
P I_{T T}=w_{T T} \overline{P V}_{T T}
$$

The control activity is calculated through equation below:

$$
P I_{C A}=w_{C A} \overline{P I}_{C A}
$$

Where the weight vectors WTT and WCA are shown on Table 1:

Table 1-Weight Vectors.

\begin{tabular}{|c|c|}
\hline wTT & {$[0.060 .080 .060 .120 .160 .120 .120 .160 .12]$} \\
\hline wCA & {$[0.100 .100 .100 .200 .100 .100 .100 .20]$} \\
\hline
\end{tabular}

Finally the total performance index can be defined as:

$$
P I_{\text {Total }}=\bar{w}_{T T} P I_{T T}+\bar{w}_{C A} P I_{C A}
$$

A weighed sum and normalization of the performance indices were done to facilitate the final calculation of the total PI. The components are normalized for each parameters between 0 (poor performance) and 1 (perfect performance) and the weights assigned to each parameter is related to the importance of each metric. In this research, the major concern is whether the aircraft is able to follow the trajectory regardless if the control activity is high, thus, more importance is given for trajectory tracking then for control activity. The value of 0.7 was used for trajectory tracking and a weight of 0.3 was assigned for control activity.

The total performance index will have different results for fixed parameter or adaptive parameter. Usually, a $\mathrm{PI}$ of above 0.6 will result in "good" trajectory tracking with small deviations from the commanded path and small oscillations. PIs ranging from 0.3 to 0.6 usually will provide poor tracking, the aircraft might deviate from the original path and may end up getting lost eventually. Lastly, total performance of below 0.3 will result in very poor tracking, where the aircraft deviates significantly from its course. Note that what "good" and "poor" means does not depend on the characteristic of the control laws. 
The trajectory tracking and control activity performance index can have a zero value assigned if the aircraft lost control or if the values exceeded the threshold. Figure 29 below shows an example of the individual metric contributions to the trajectory tracking and control activity indices. The respective cutoff performance indices values for trajectory tracking and control activity can be seen on Table 2 .

Table 2- Cutoff Values for Performance Indices

\begin{tabular}{|l|l|}
\hline Cutoff_PI Trajectory Tracking & {$\left[\begin{array}{l}50505010101055 \text { 5] meters } \\
\hline \text { Cutoff_PI Control Activity }\end{array}\right][0.50 .50 .520100100100100]$} \\
\hline
\end{tabular}
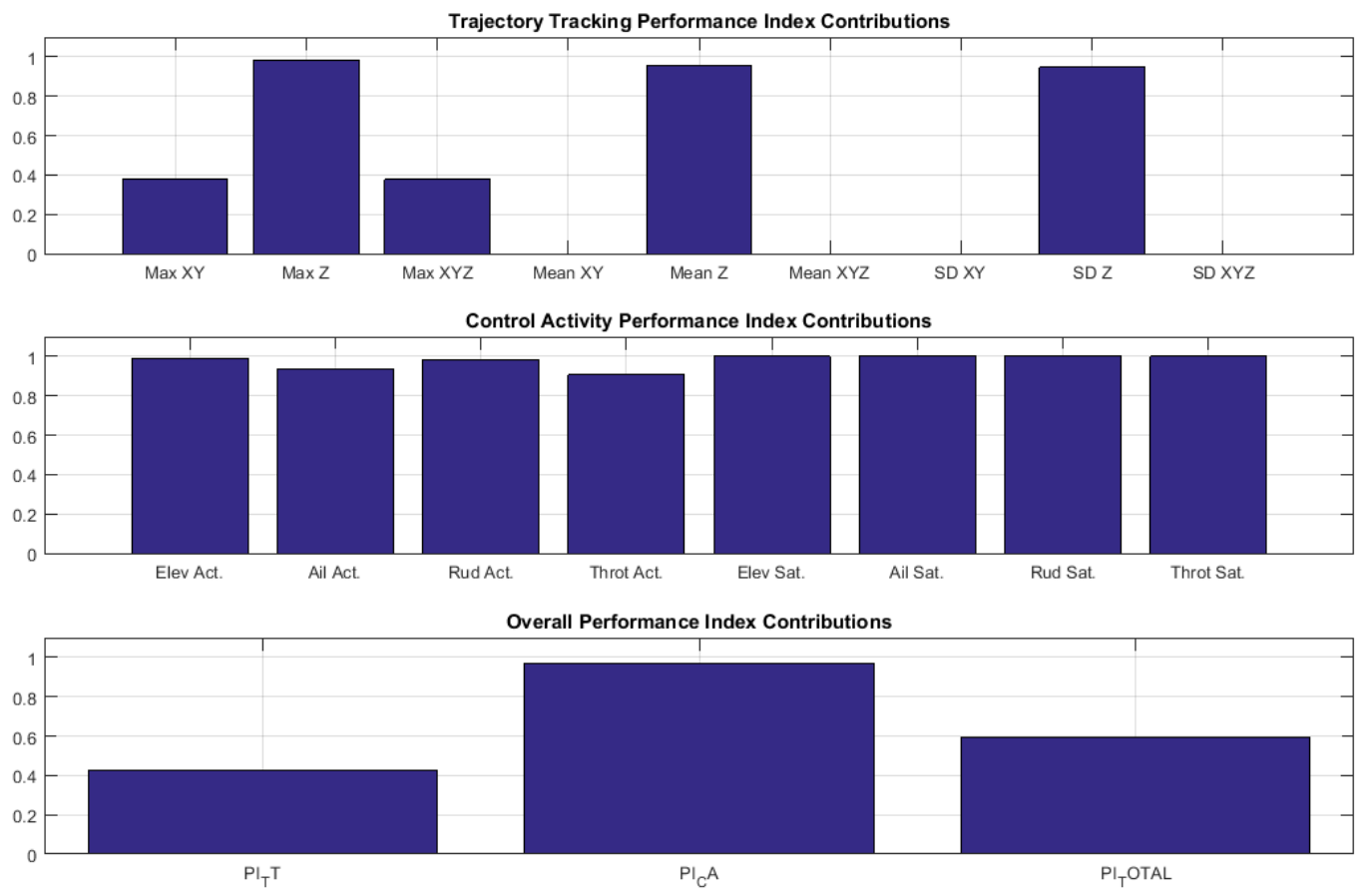

Figure 29-Total Performance Index Contributions. 


\section{RESULTS AND ANALYSIS}

In this chapter, an analysis of UAV trajectory tracking performance is performed by assessing the effects of the most relevant atmospheric phenomena: wind, turbulence, gusts and wind shear. The evaluation of trajectory tracking performance relies on composite performance indices based on tracking error and control activity, as described in Chapter 4. It should be noted for control activity evaluation, the higher the bar, the less the controller was working. In other words, the more the controller was working to keep the aircraft on its path, the lower the score it was assigned. When interpreting the values of the control activity index, it should be considered that high values are indicative of "good" performance only in conjunction with good tracking. High values may be assigned when the controller is producing no control compensation while moving away from the commanded trajectory. Values close to zero of the trajectory tracking error index show, obviously, poor performance; however, they are not always an indication of a crash or complete departure from commanded trajectory. To make distinction among such situations, further inspection of aircraft states is necessary. The presence of atmospheric phenomena is considered as "abnormal condition". A sensitivity analysis with respect to the severity and other characteristics of these phenomena is performed through comparison with nominal conditions.

The aircraft used for the simulations is the WVU-YF22 aircraft [64]. Abnormal conditions were simulated using the wind models developed in this research effort, with exception of the turbulence model that was already available in the WVU simulation environment. The respective trajectories used for the simulations were presented in the previous chapter.

This chapter also includes a limited discussion of the additional UAV performance challenges under actuator failures. Locked elevator and aileron scenarios were considered.

It should be noted that the large number of factors and levels of severity/magnitude produced a large number of test cases. While all of them are considered in the discussion, only the most representative plots are included in this chapter, the rest are presented in the appendix.

\subsection{Experimental Design}

In this experiment, there were six main factors analyzed: control laws, commanded trajectory, constant wind, wind gust, wind shear, and actuator condition. A summary of all factors and levels of severity is presented from Table 3 to Table 7. The primary outcomes of the experiment were the relative response of the aircraft capability of following the trajectory with 
minimum errors and effort of the controller. This is given in terms of performance index values for trajectory tracking and control activity, respectively.

According to the specifications described in Table 3, Table 4 and Table 5, the number of cases investigated for constant wind were F1x F2x F3 $(2 \times 5 \times 36)$ for a total of 360 cases. The wind direction is given in Earth axes and was plotted against the performance evaluated, that being control activity or trajectory tracking error index. The magnitude was defined based on local wind speed (West Virginia) [68]. The highest magnitude was defined as $25 \mathrm{kts}$ and low magnitude was defined as $10 \mathrm{kts}$. In this research effort, the effects of turbulence were evaluated when the aircraft is experiencing constant wind. No mathematical model was created in this project for turbulence, instead, a previous model available in the WVU simulation environment was used in which the mathematical model was created based on the Dryden model. The turbulence intensity for low constant wind is $1 \%, 2.5 \%$ and $4 \%$ (low, medium and severe turbulence respectively), and for high speed constant wind case, the turbulence intensity is $0.4 \%, 1 \%$ and $1.6 \%$ (low, medium and severe turbulence respectively).

To analyze the wind gust phenomenon the factors considered were F1xF2xF4 $(2 \times 3 \times 48)$ (see Table 3, Table 4 and Table 6) for a total of 288 cases. The results were plotted against performance index of control activity or trajectory tracking. The wind gust direction shown is related to body axis for better analysis of the gust effects. The magnitude of the gust was defined experimentally based on references listed in Chapter 2. The highest value (40 kts) was assigned to be the one where the trajectory tracking starts to become poor but the aircraft will still manage to follow the commanded trajectory. The lowest magnitude is defined as half of the highest value. Gust duration was defined as 20 seconds based on references [33, 34]. This was used for long duration and 10 seconds for short duration. Simulations were performed with the gust occurring in the middle of a turn or straight flight segment, and for the 3D s turns trajectory, this occurs during climb.

In order to analyze the wind shear effect, the factors simulated were F1xF2xF5 $(2 \times 3 \times 36)$ for a total of 216 different scenarios (see Table 3, Table 4 and Table 7). The levels of severity of the shear in each direction was determined experimentally through simulation, with highest value possible where the aircraft doesn't lose control and lowest being half that value. In comparison with look up tables found in [45], for vector component along $X$ axis the value determined experimentally is about half of the maximum look up table value. For vector component in $Z$, the maximum value determined experimentally is close to the values encountered in the look up tables. There was no reference values found for vector component 
along $\mathrm{Y}$ direction, thus, if compared to values found for vector components along $\mathrm{X}$ direction, the experimental values are close to the values of the look up tables. For each vector component, the gradient direction was varied only in $\mathrm{X}, \mathrm{Y}$ and $\mathrm{Z}$ direction with respect to that component. Constant wind had to be input for the simulation, thus, the magnitude used was $15 \mathrm{kts}$. The direction of the constant wind was defined in Earth axes and is $\Psi_{\mathrm{w}}=180^{\circ}, \Psi_{\mathrm{w}}=90^{\circ}$ and $\theta_{\mathrm{w}}=-30^{\circ}$ for $X, Y$ and $Z$ respectively (only one wind direction was simulated for each vector). The wind shear was simulated occurring in the middle of turn or middle of straight flight path for a duration period of 20 seconds. It should be noted that for the 3D s turns trajectory, this occurs during climb.

A few simulations were done involving wind phenomena in addition to actuator failures. According to Table 8, a total of 18 cases (F1XF6) were investigated were the failure affected the left and right ailerons. The failures occurred within 5 seconds into the simulation and continued for the duration of the simulation. The aircraft only encountered wind gust during turn flight path for 10 seconds (duration of gust). To study the sensitivity of the controllers, the aileron is locked at $5^{\circ}$ degrees (medium severity level failure) for all cases in an attempt to expose the effects of the failure without making the aircraft lose control. The constant wind magnitude was defined as low (10 kts) and the wind gust magnitude was defined as $20 \mathrm{kts}$. All cases were investigated for oval trajectory only.

It should be noted that all the simulation scenarios considered were simulated only once. While it is well understood that when using actual experimental data, repeated similar tests are necessary for statistical significance, inserting additional variability and perturbations into the simulation was considered beyond the purpose of the study. The turbulence model has a random component; however, the statistical properties of the random process are consistently the same in similar simulation tests.

Table 3 - Control Laws

\begin{tabular}{|c|c|}
\hline \multirow{2}{*}{ F1= Control Laws } & Fixed Parameter Position PID \\
\cline { 2 - 2 } & Adaptive Parameter Position PID \\
\hline Total & 2 \\
\hline
\end{tabular}


Table 4- Commanded Trajectories

\begin{tabular}{|c|c|}
\hline \multirow{4}{*}{ F2= Commanded Trajectory } & Oval (2x minimum turning radius) \\
\cline { 2 - 2 } & Figure 8 (2x minimum turning radius) \\
\cline { 2 - 2 } & 3D S turns (2x minimum turning radius) \\
\cline { 2 - 2 } & Dubins \\
\cline { 2 - 2 } & Clothoid \\
\hline Total & 5 \\
\hline
\end{tabular}

Table 5- Constant Wind Factors and Levels

\begin{tabular}{|c|c|}
\hline \multirow{2}{*}{ F3= Constant Wind (this set has 3 factors) } \\
\hline \multirow{2}{*}{ Wind Magnitude } & Low $=10 \mathrm{kts}$ \\
\cline { 2 - 2 } Wind Direction (Earth Axes) & High $=25 \mathrm{kts}$ \\
\cline { 2 - 2 } & $\Psi_{\mathrm{w}}=180^{\circ}$ (head), $\Psi_{\mathrm{w}}=90^{\circ}$ (left) \\
\hline Turbulence on All 3 Axes & $\theta_{\mathrm{w}}=0^{\circ}$ (tail), $\theta_{\mathrm{w}}=30^{\circ}$ (up), $\theta_{\mathrm{w}}=-30^{\circ}$ (down) \\
\hline Total & $2 \times 6 \times 3=36$ \\
\hline
\end{tabular}

Table 6 - Wind Gust Factors and Levels

\begin{tabular}{|c|c|}
\hline \multicolumn{2}{|c|}{$\mathrm{F} 4$ = Wind Gust (this set has 4 factors) } \\
\hline \multirow{2}{*}{ Gust Magnitude } & Low $=20 \mathrm{kts}$ \\
\cline { 2 - 2 } & High $40 \mathrm{kts}$ \\
\hline \multirow{2}{*}{ Gust Duration } & Short $=10 \mathrm{~s}$ \\
\cline { 2 - 2 } & Long $=20 \mathrm{~s}$ \\
\hline Gust Direction (Earth axes) & $\begin{array}{c}\theta_{\mathrm{G}}=-90^{\circ} \text { (down), } \theta_{\mathrm{G}}=90^{\circ} \text { (up), } \Psi_{\mathrm{G}}=0^{\circ} \text { (tail) } \\
\Psi_{\mathrm{G}}=180^{\circ} \text { (head), } \Psi_{\mathrm{G}}=90^{\circ} \text { (left), } \Psi_{\mathrm{G}}=270^{\circ} \text { (right) }\end{array}$ \\
\hline Gust Location & $\begin{array}{c}\text { Middle of turn } \\
\text { Middle or straight segment }\end{array}$ \\
\hline Total & $2 \times 2 \times 6 \times 2=48$ \\
\hline
\end{tabular}


Table 7- Wind Shear Factors and Levels

\begin{tabular}{|c|c|}
\hline \multicolumn{2}{|c|}{ F5 $=$ Wind Shear (this set has 6 factors) } \\
\hline \multirow{3}{*}{ Wind Magnitude } & Gradient "X" - Small $=0.0125$, large $=0.025 \quad(\mathrm{~m} / \mathrm{s}) / \mathrm{m}$ \\
\hline & Gradient "Y" -Small $=0.0175$, large $=0.035(\mathrm{~m} / \mathrm{s}) / \mathrm{m}$ \\
\hline & Gradient "Z" - Small $=0.01$, large $=0.02 \quad(\mathrm{~m} / \mathrm{s}) / \mathrm{m}$ \\
\hline Wind Vector Component & $\mathrm{X}_{\mathrm{cw}}, \mathrm{Y}_{\mathrm{cw}}$ and $\mathrm{Z}_{\mathrm{cw}}$ \\
\hline Gradient Direction & $X_{w}, Y_{w}$ and $Z_{w}$ \\
\hline \multirow{2}{*}{ Wind Shear Location } & Middle of turn \\
\hline & Middle of straight segment \\
\hline Wind Shear Duration & $20 \mathrm{~s}$ \\
\hline Constant Wind Magnitude & $15 \mathrm{kts}$ \\
\hline Total & $2 \times 3 \times 3 \times 2 \times 1 \times 1=36$ \\
\hline
\end{tabular}

Table 8- Actuator Failures Factors and Levels

\begin{tabular}{|c|c|}
\hline \multicolumn{2}{|c|}{ F6= Failures (this set has 6 factors) } \\
\hline Wind Direction and Gust Direction (Earth Axes) & $\begin{array}{c}\Psi_{\mathrm{w}}=180^{\circ} \text { (head), } \Psi_{\mathrm{w}}=90^{\circ} \text { (left), } \Psi_{\mathrm{G}}= \\
180^{\circ} \text { (head) }\end{array}$ \\
\hline Wind Magnitude & \begin{tabular}{c}
$10 \mathrm{kts}$ \\
\hline Aileron and Stabilator Failures
\end{tabular} \\
\hline Gust Magnitude & Ailerons=Left and right, stabilator= (left) \\
\hline Gust Location & $3 \times 1 \times 3 \times 1 \times 1=9$ \\
\hline Total & Middle of turn \\
\hline
\end{tabular}

\subsection{Control Laws Analysis}

There were two controllers used for the analysis of the wind phenomena, the fixed parameter PPID and the adaptive parameter PPID. After all simulations were concluded, a trend was observed throughout all of the cases regarding different types of wind phenomena. Overall, the adaptive controller exhibits, in the majority of the cases, a greater performance index than the fixed parameter controller. It should be noted that, as it is shown in Figure 30 and Figure 31 below, the adaptive controller has a much higher tracking error performance under nominal conditions (around 0.9) compared to the fixed parameter one (around 0.5). On the other hand, 
the index values for the adaptive parameter controller dropped significantly in the presence of wind, while the fixed parameter controller exhibited a much smaller decrease.

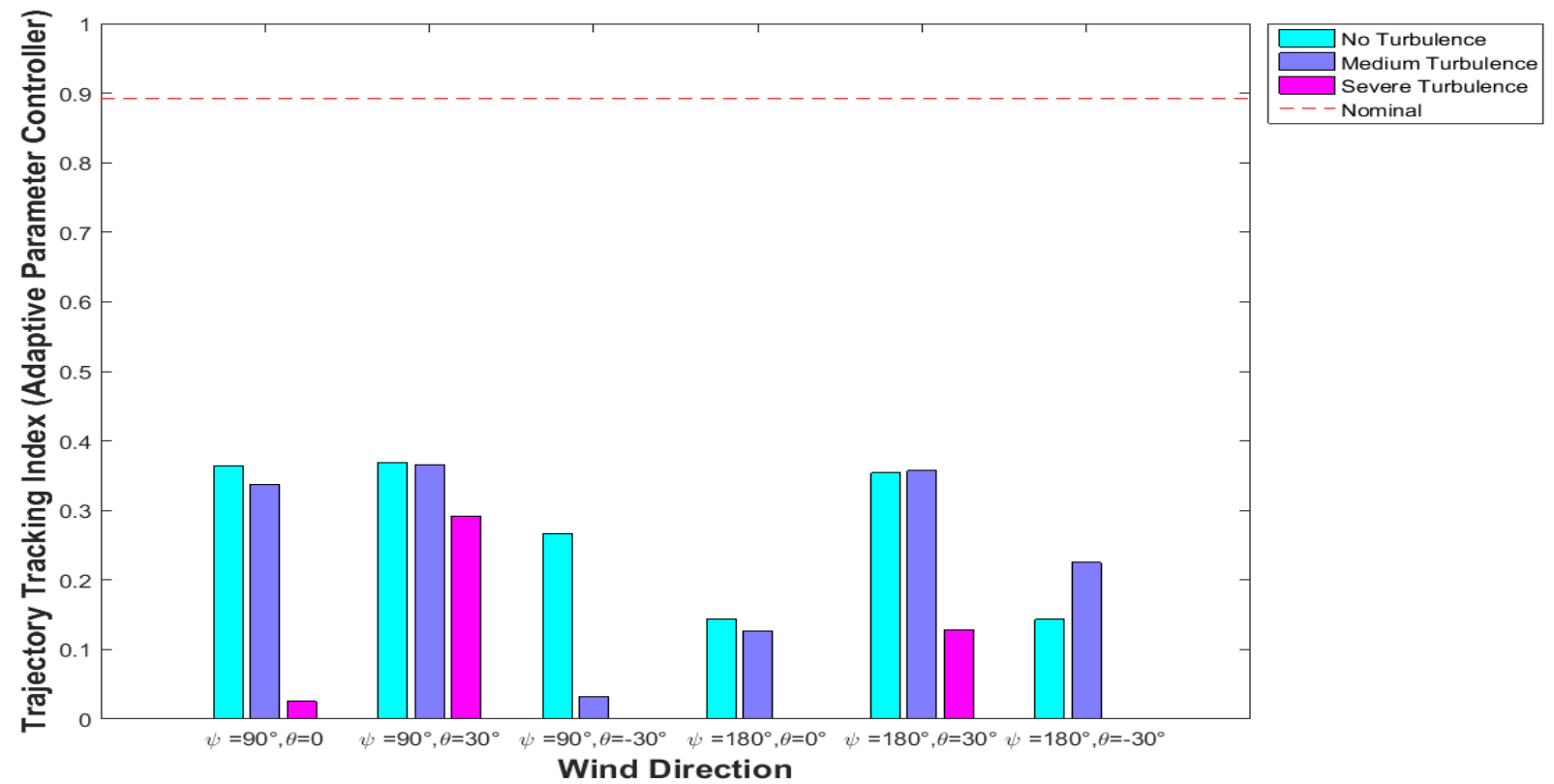

Figure 30- Tracking Index for Oval Trajectory Under Strong Constant Wind (Adaptive PPID).

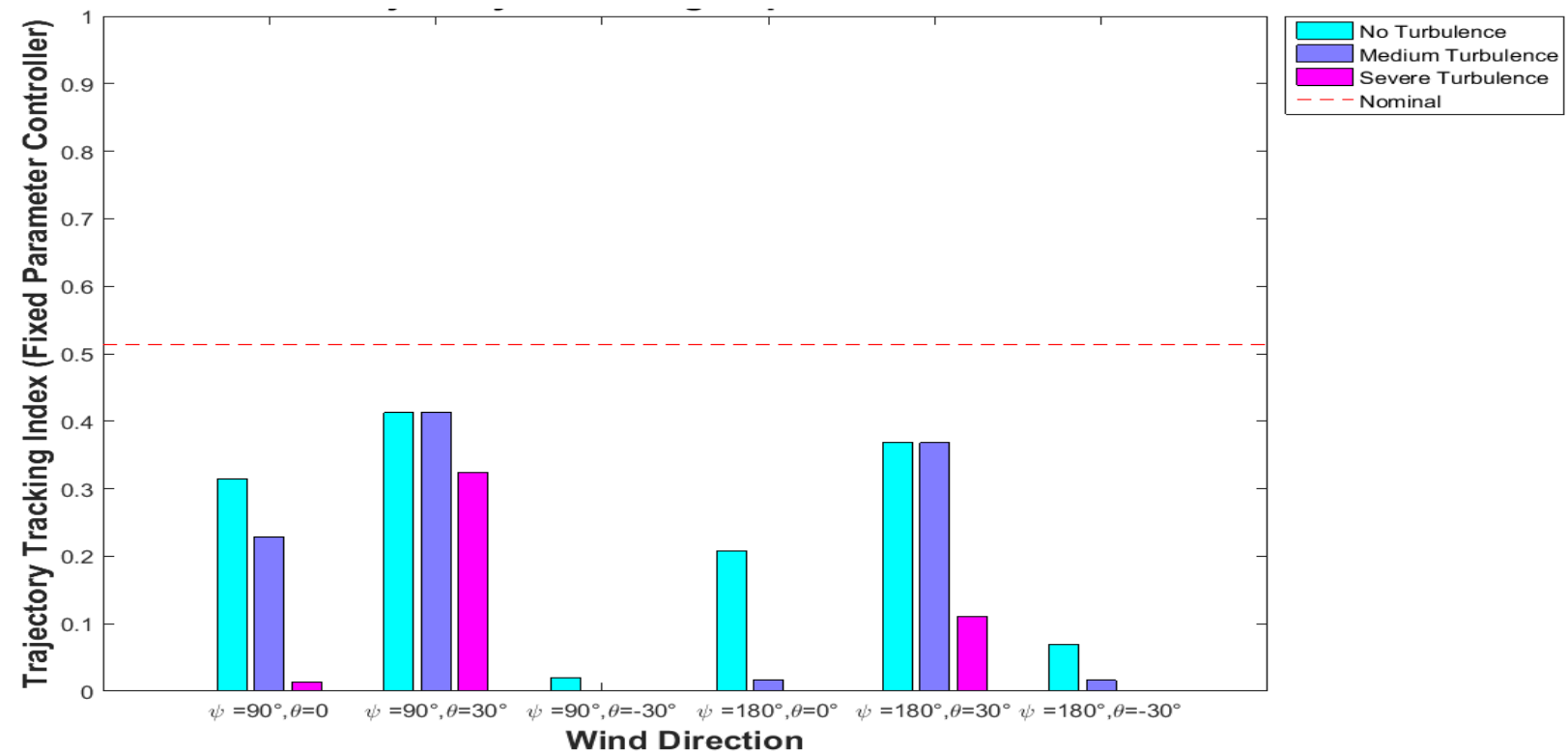

Figure 31- Tracking Index for Oval Trajectory Under Strong Constant Wind (Fixed PPID).

Since adaptive controllers are expected to have a higher performance then fixed parameter counterparts, a different verification test was performed for confirmation. For this, it was used a more advanced adaptive controller and its respective fixed parameter version of the 
nonlinear dynamic inversion (NLDI) extended type [4]. The simulation was performed for only one case just to confirm the trend seen about the degradation of the adaptive controller. The NLDI adaptive controller behaved similar to the adaptive PPID exhibiting a high degradation. As it can be seen in Figure 32 (high speed constant wind), although the performance of the adaptive version is still higher under constant wind, both controllers performed poorly. The index is zero for fixed parameter under high turbulence and the controller oddly performs better under medium turbulence than under no turbulence at all. The adaptive parameter controller seemed to be indifferent to no turbulence or medium turbulence. On the other hand, the performance index was very low for severe turbulence. The values for nominal conditions of fixed and adaptive parameter controller overlap for the NLDI controller. These results suggest that the benefits of adaptive control in still atmosphere do not necessarily transfer under wind conditions. Thus, the trend seen about the degradation of the adaptive PPID was confirmed.

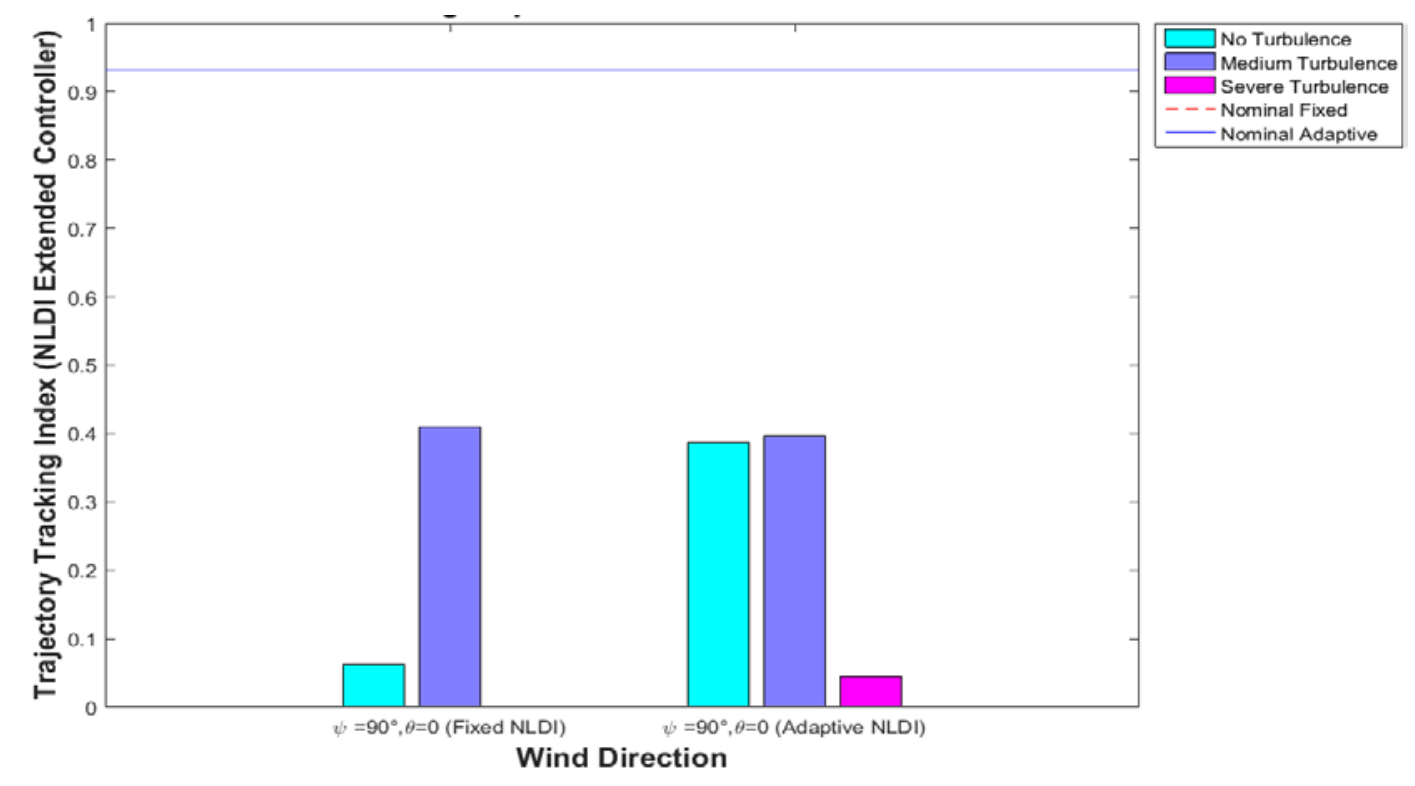

Figure 32- Tracking Index for Oval Trajectory Under Strong Constant Wind for NLDI Extended (Fixed vs Adaptive).

Another trend was observed for the control activity. The control activity increases under wind conditions as expected. However, there is small difference between the adaptive and fixed parameter versions of the control laws. Also, the control activity index appeared to be insensitive to wind magnitude. This difference is very small, barely perceptible by looking at the plots. Since the adaptive controller is constantly changing gains to compensate for abnormal conditions, it was expected that it would exhibit more activity under wind influence than the fixed parameter controller. However, this was not the case in this study as can be seen in Figure 33 and Figure 34. 
The control activity increased as turbulence levels increased, but the difference between control activity under wind shear and wind gust is small. This is shown in Figure 34, Figure 35 and Figure 43.

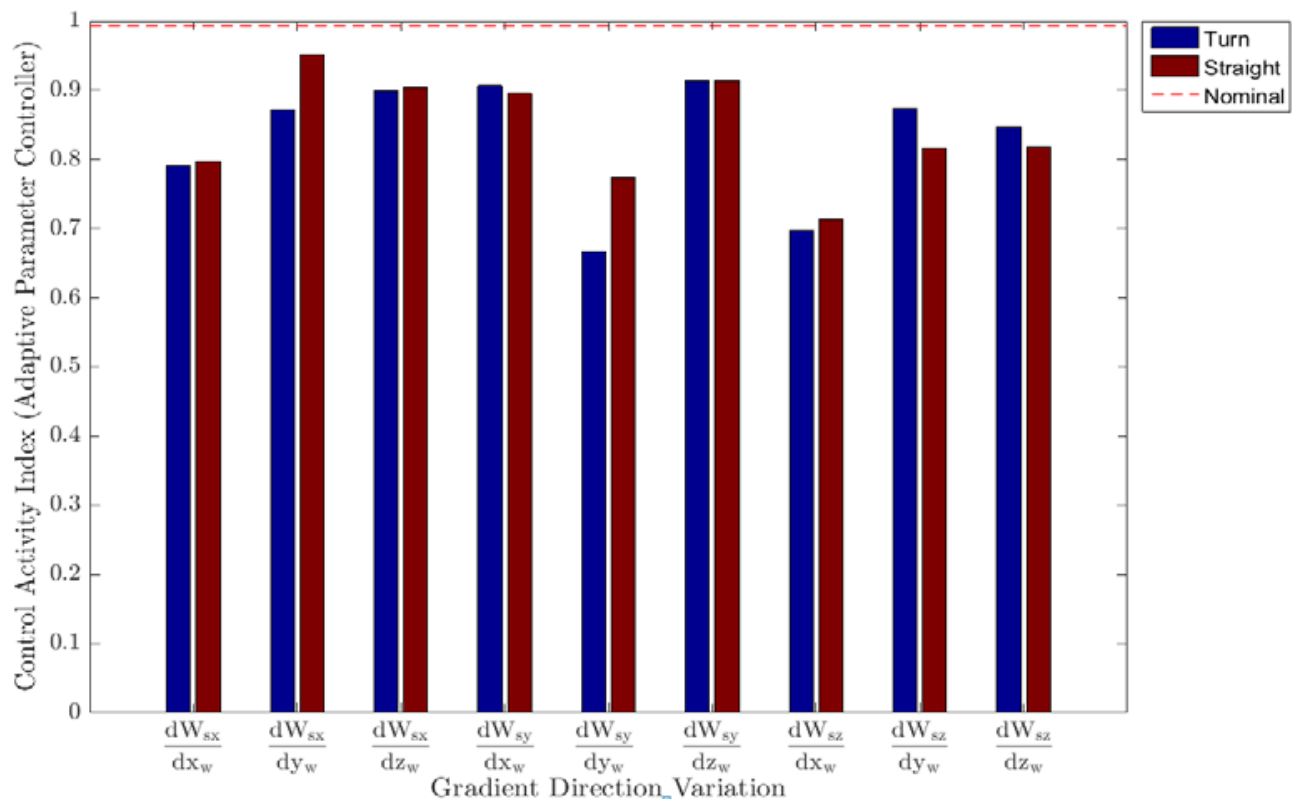

Figure 33- Activity Index for Figure 8 Trajectory Under Strong Wind Shear (Adaptive PPID).

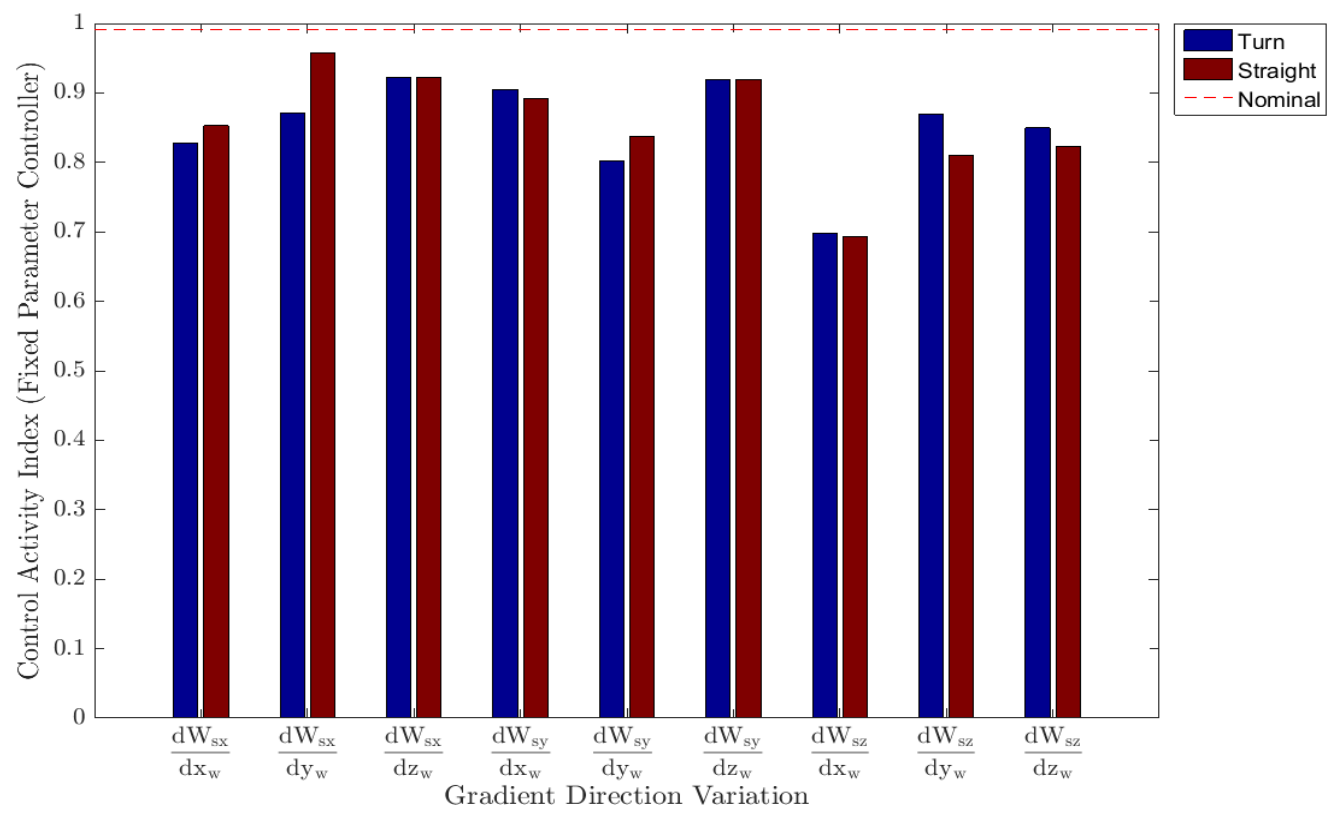

Figure 34- Activity Index for Figure 8 Trajectory Under Strong Wind Shear (Fixed PPID).

Even with the tracking performance index of the NLDI Extended controller not being much better than the PPID controllers, the control activity is a lot higher if compared with trends seen 
previously with respect to the PPID controllers. The lowest index for fixed PPID controller is around 0.7 (Figure 35), and the index values for control activity for NLDI controllers are a lot worse (around 0.37) as presented in Figure 36. Oddly, the results show that under medium turbulence, there is less effort of the controller, thus, a higher index is assigned for medium turbulence than for no turbulence or severe turbulence.

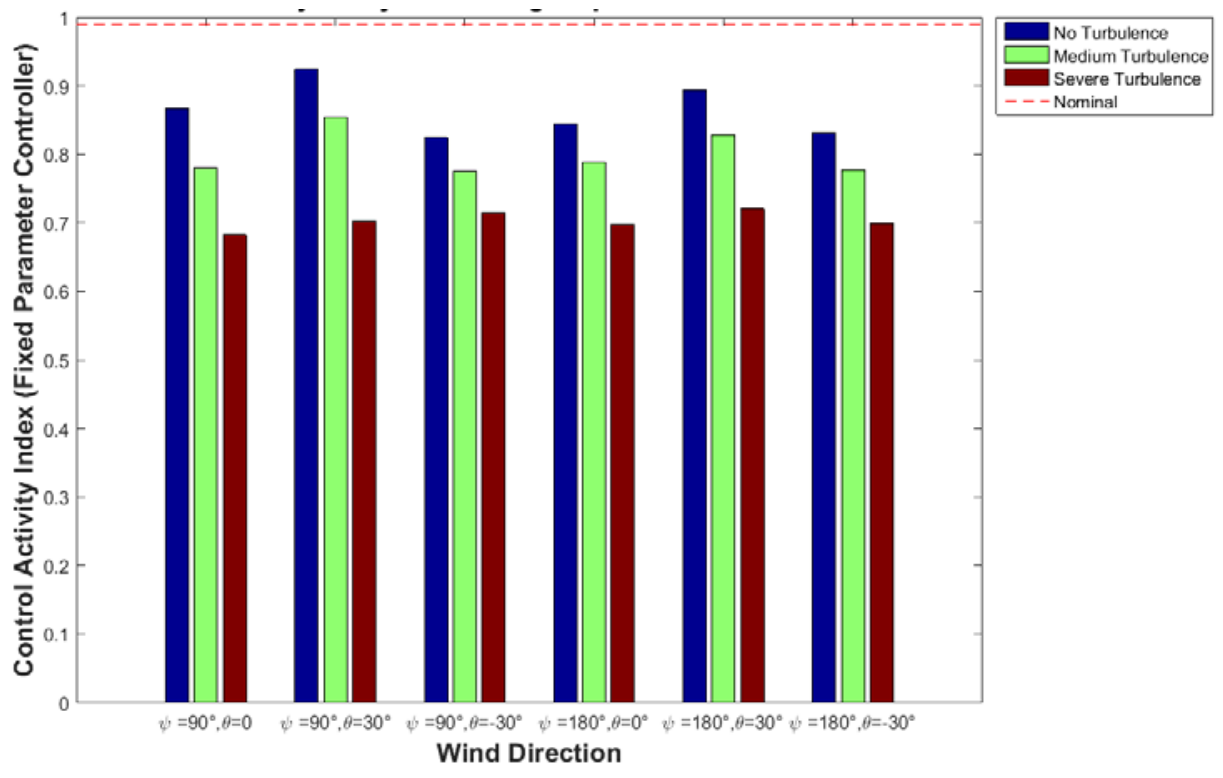

Figure 35- Activity Index for Oval Trajectory Under Strong Constant Wind (Fixed PPID).

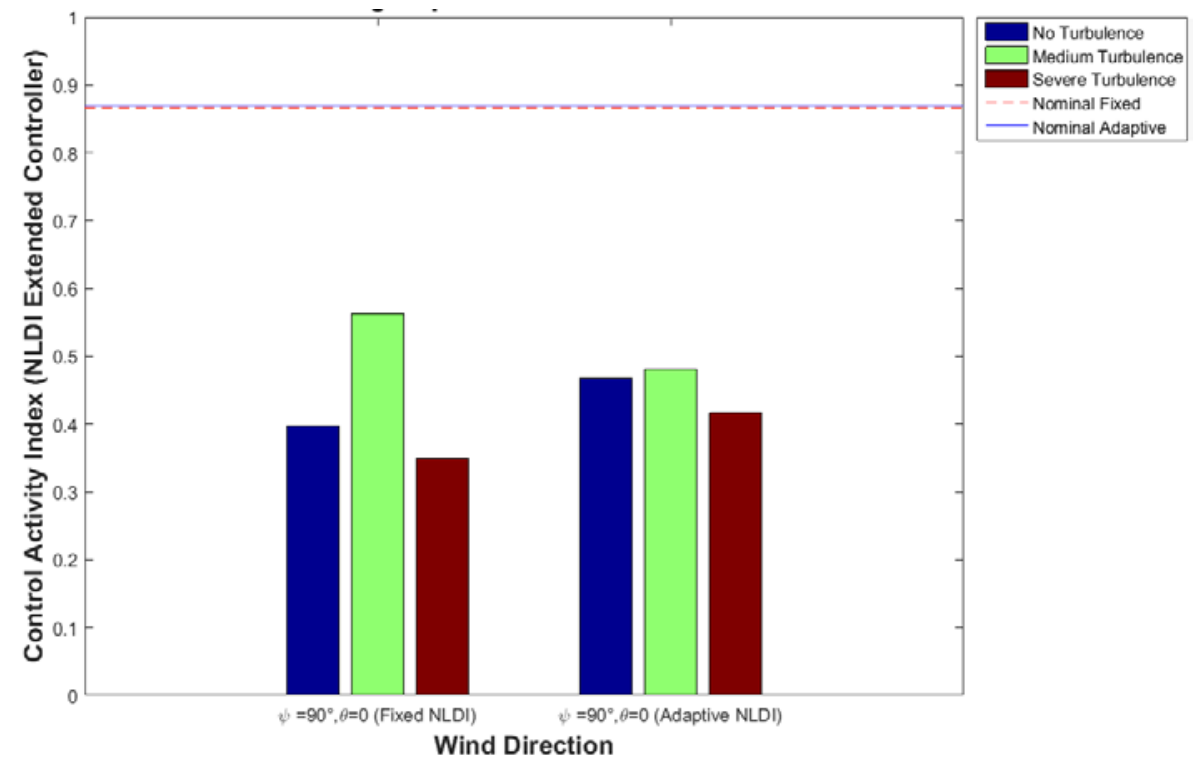

Figure 36- Activity Index for Oval Trajectory Under Strong Constant Wind for NLDI Extended (Fixed Vs Adaptive). 


\subsection{Commanded Trajectory Analysis}

The commanded trajectory factor also has an impact on the results. In this study, as previously discussed, five different types of trajectories were simulated: clothoid, Dubins, figure 8, oval and 3D S turns. The results for clothoid, Dubins, and 3D s turns are presented in Figure 37, Figure 38 and Figure 39.

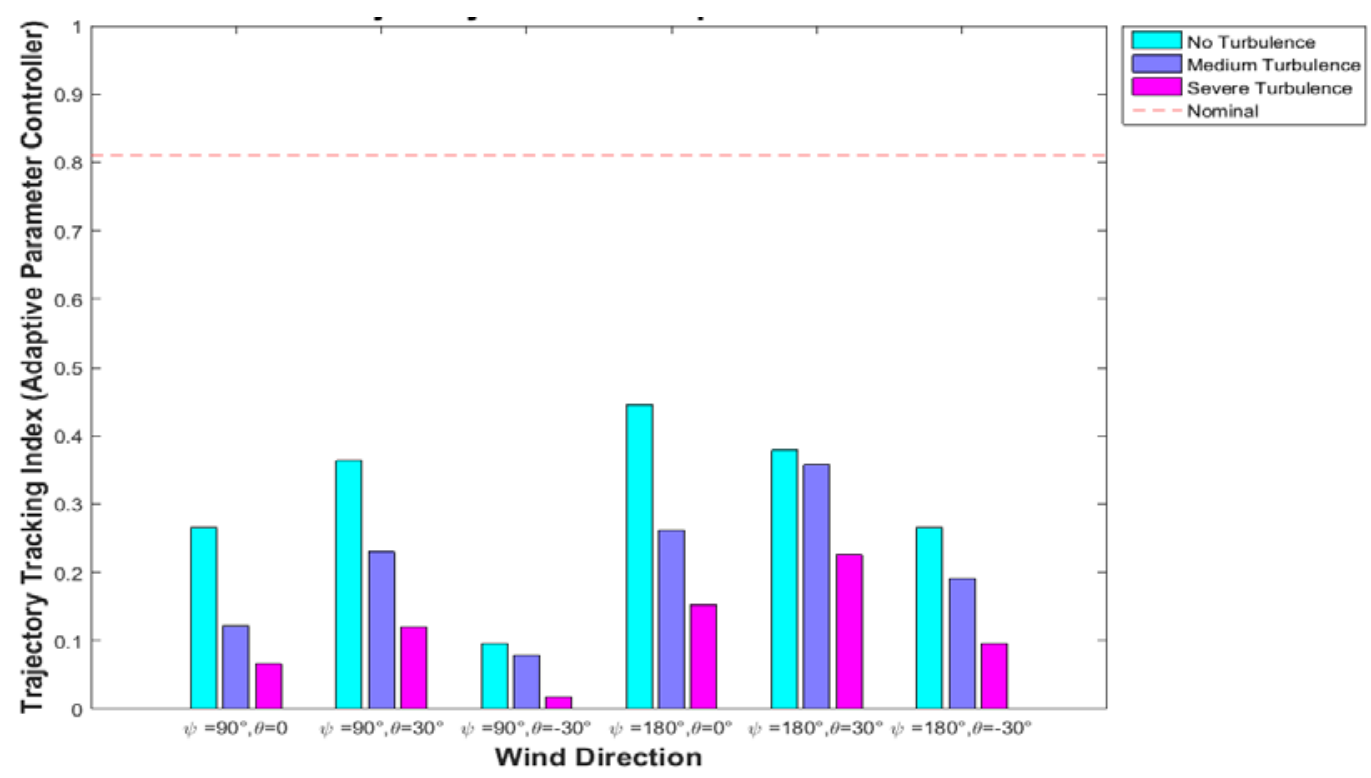

Figure 37- Tracking Index for Clothoid Trajectory Under Low Constant Wind (Adaptive PPID).

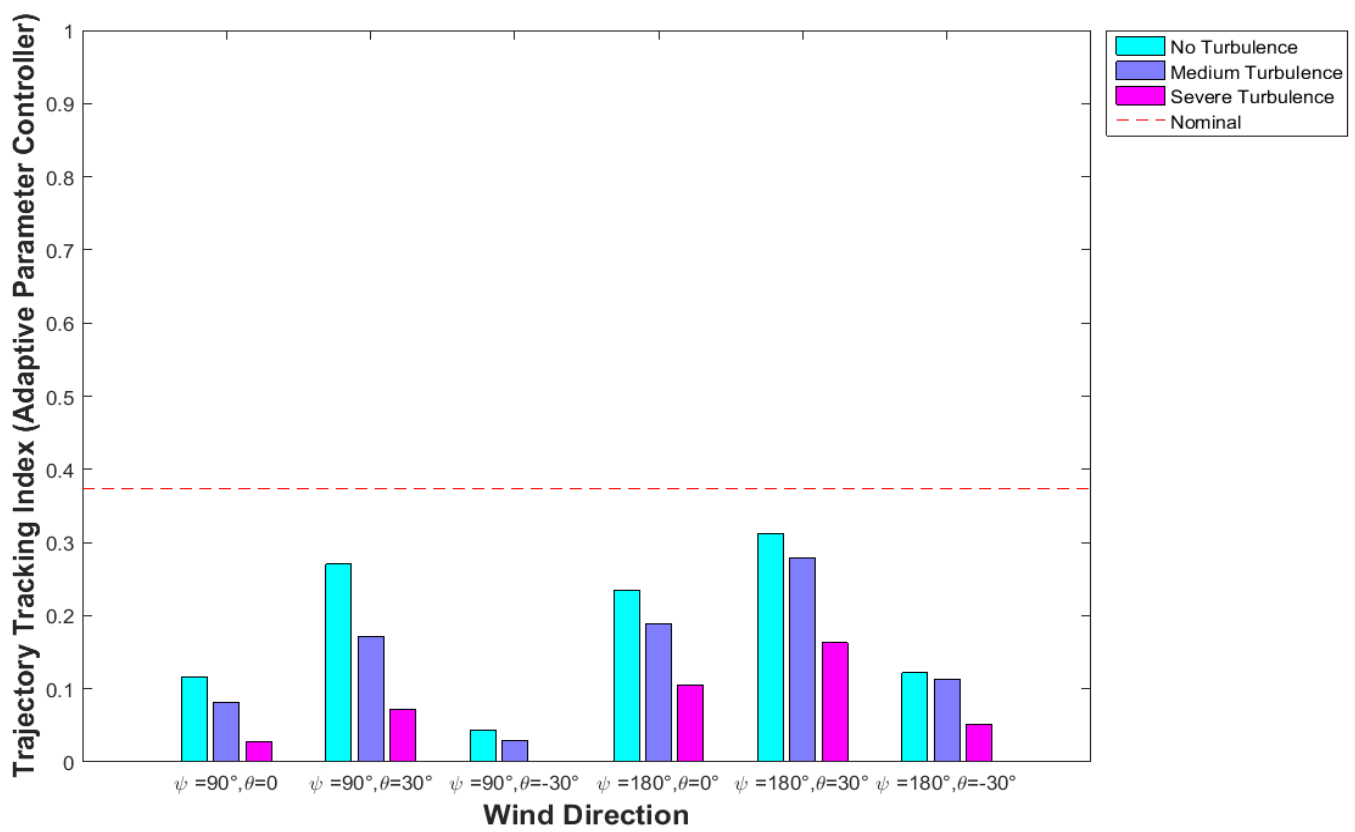

Figure 38- Tracking Index for Dubins Trajectory Under Low Constant Wind (Adaptive PPID). 


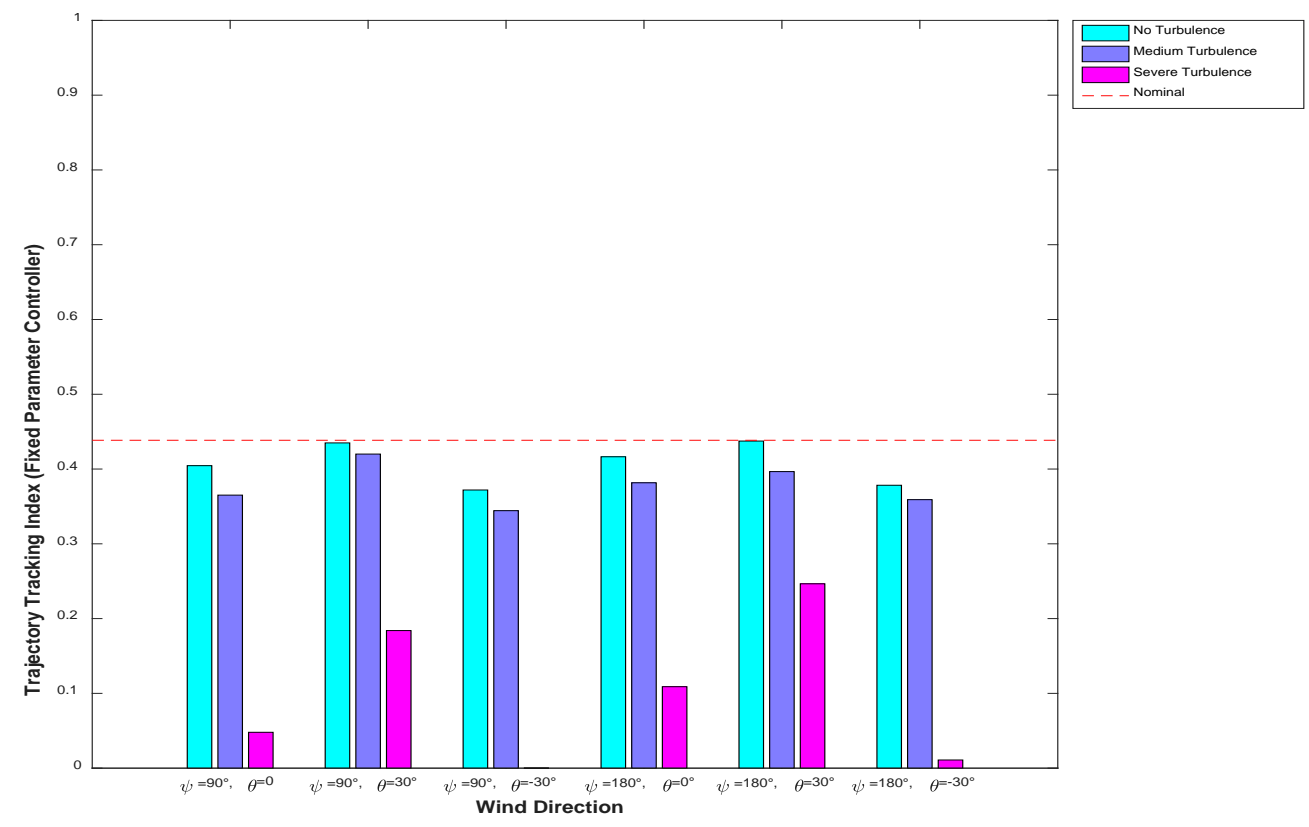

Figure 39- Tracking Index for 3D S Turns Trajectory Under Low Constant Wind (Fixed PPID).

Depending on the trajectory path, the performance index for nominal conditions decreased considerably as in the case of Dubins and 3D S turns if compared to clothoid trajectory. This is due to the superior smoothness of the clothoid path. For trajectory tracking, the performance index for nominal conditions for adaptive controller in the Dubins trajectory, dropped to about half the value obtained for clothoid trajectory. The 3D s turns also presented low performance index for nominal condition.

Another peculiarity found only in the Dubins trajectory is that the adaptive and fixed PPID controllers have a very similar tracking index under nominal conditions (less than 0.4). Figure 40 illustrates this peculiarity.

For the 3D s turns, the performance indices presents smother variations and are closer to the nominal conditions for the adaptive and the fixed parameter controllers under wind gust and also wind shear. This trend can be seen in Figure 41 and Figure 42. 


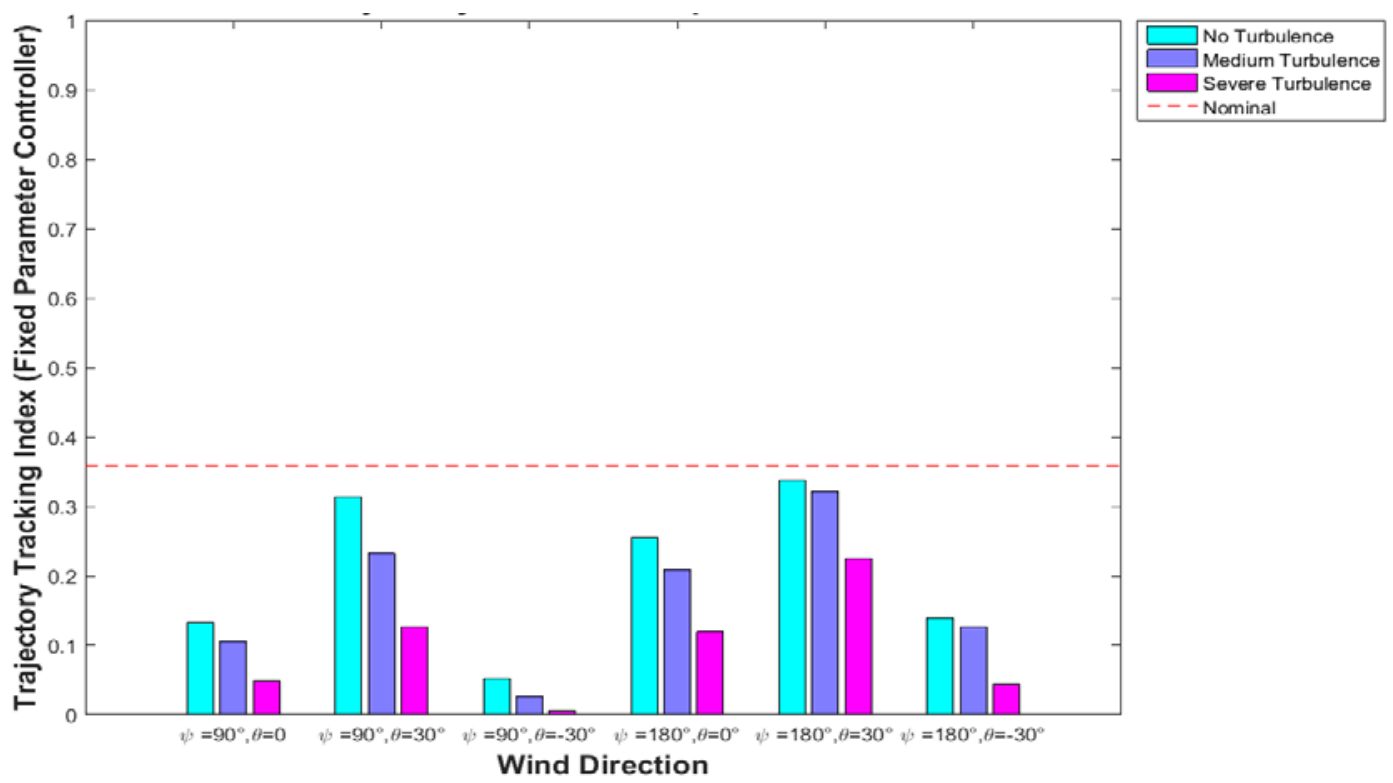

Figure 40-Tracking Index for Dubins Trajectory Under Low Constant Wind (Fixed PPID).

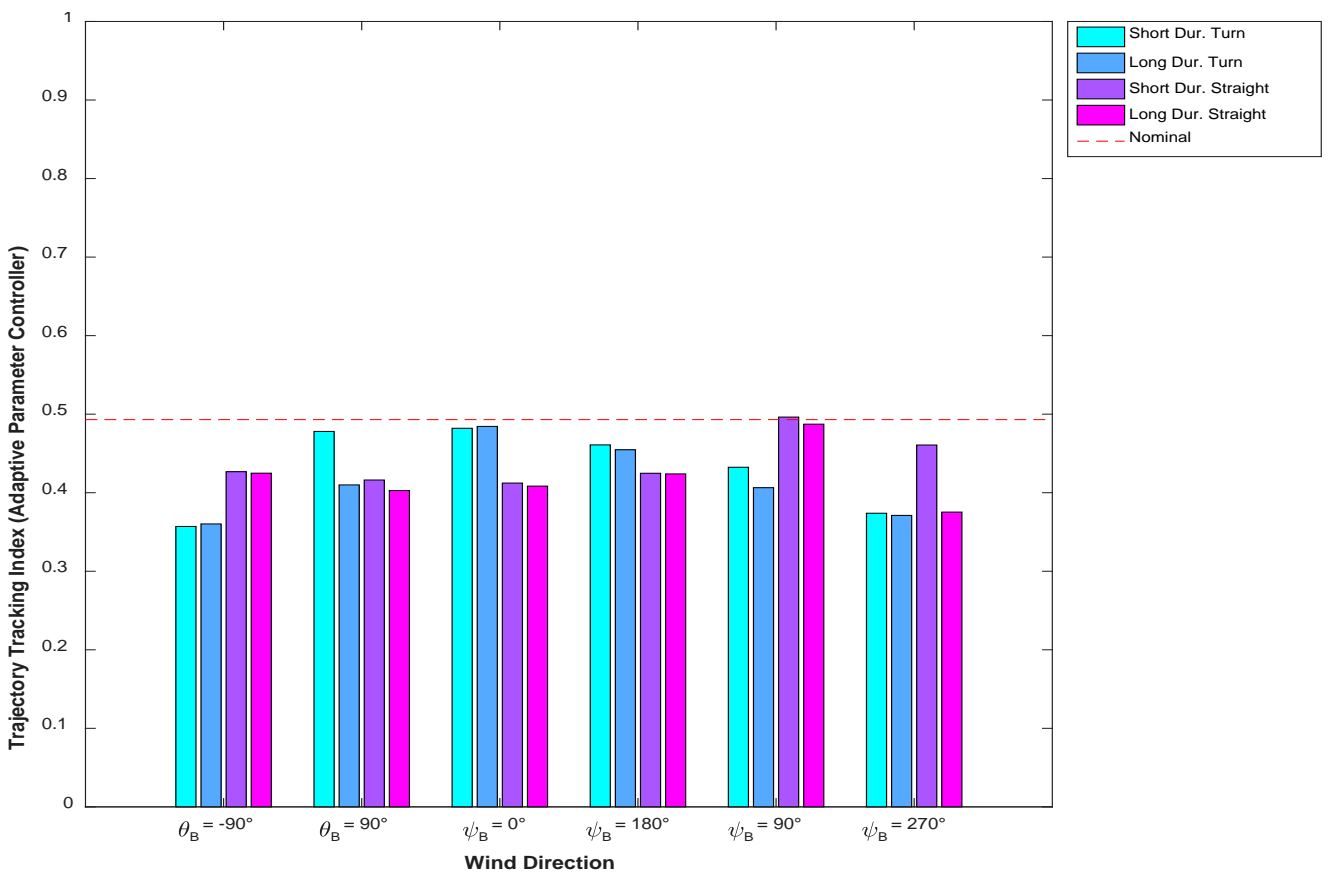

Figure 41- Trajectory Index for 3D S Turns Under Low Wind Gust (Adaptive PPID). 


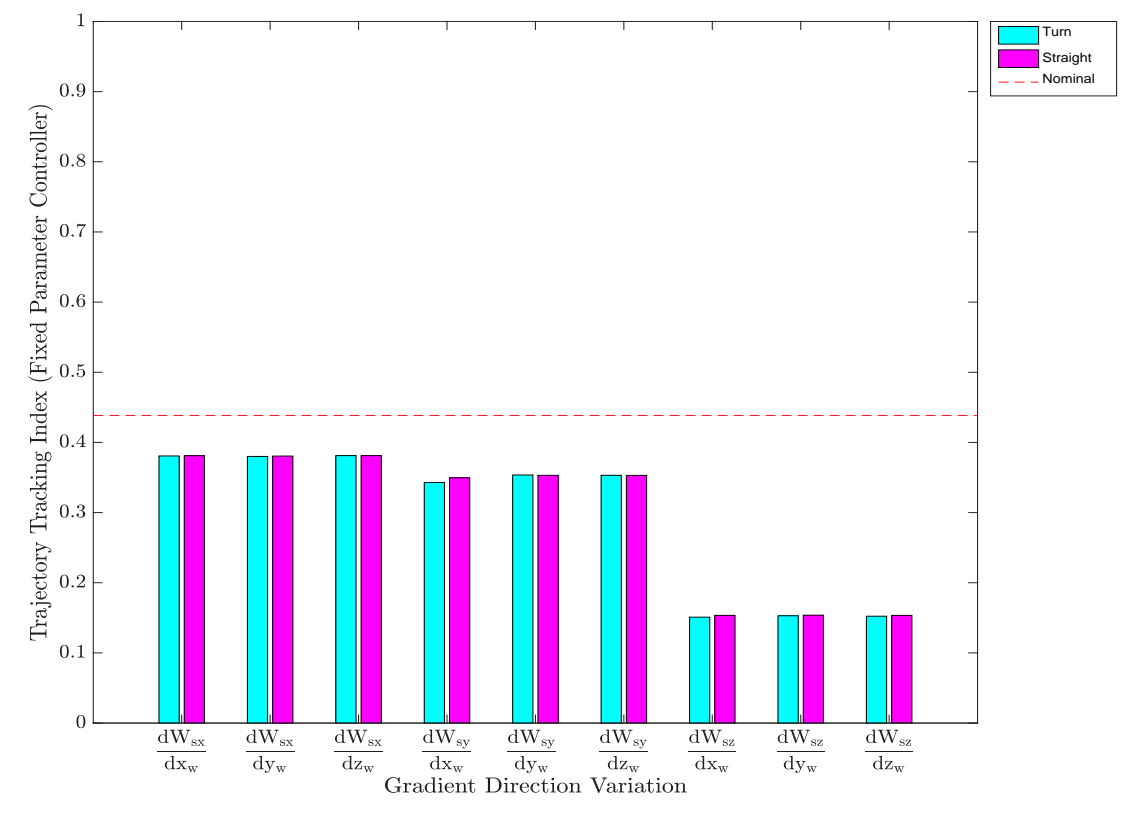

Figure 42- Trajectory Index for 3D S Turns Under Low Wind Shear (Fixed PPID).

There was not much change found for control activity related to different trajectories. Thus, the performance index was almost equivalent for all trajectories. This trend is shown in Figure 43 and Figure 44.

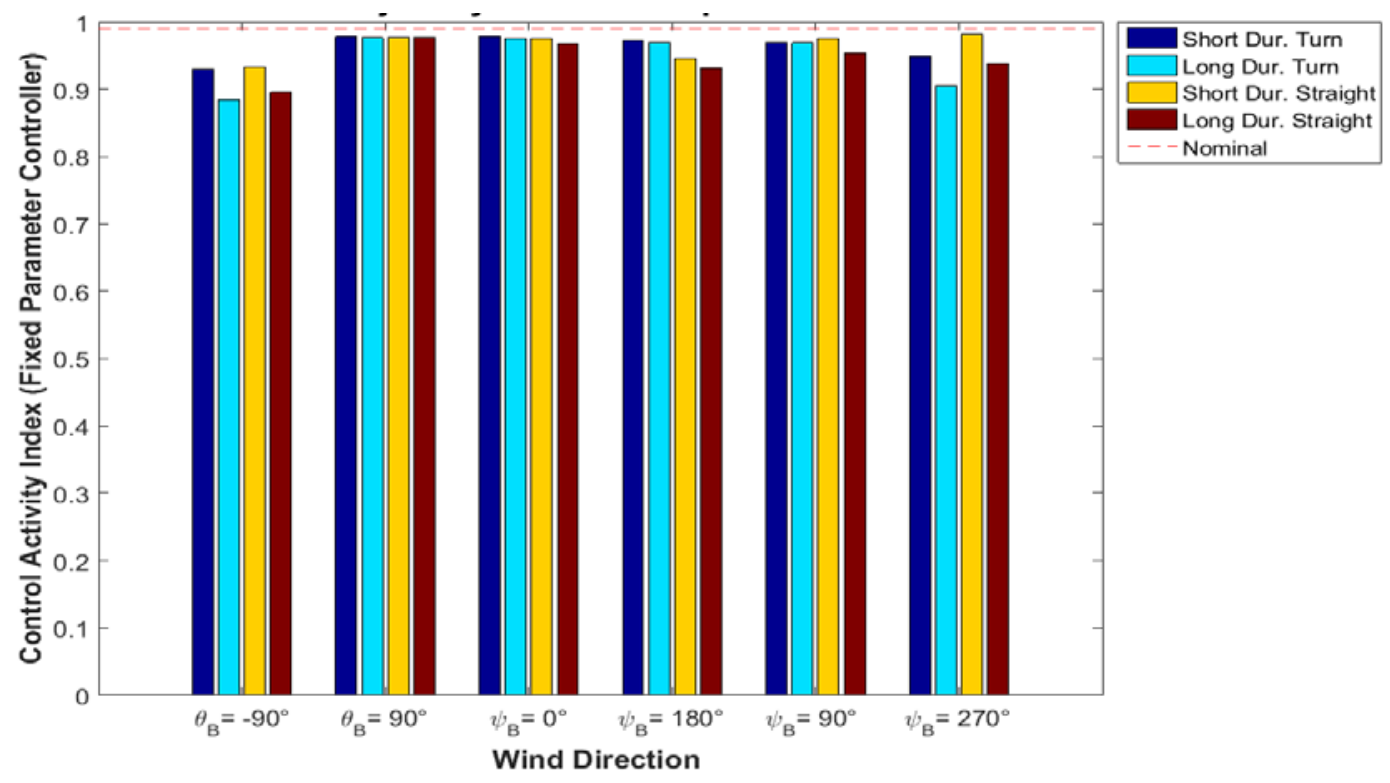

Figure 43- Activity Index for Oval Trajectory Under Low Wind Gust (Fixed PPID). 


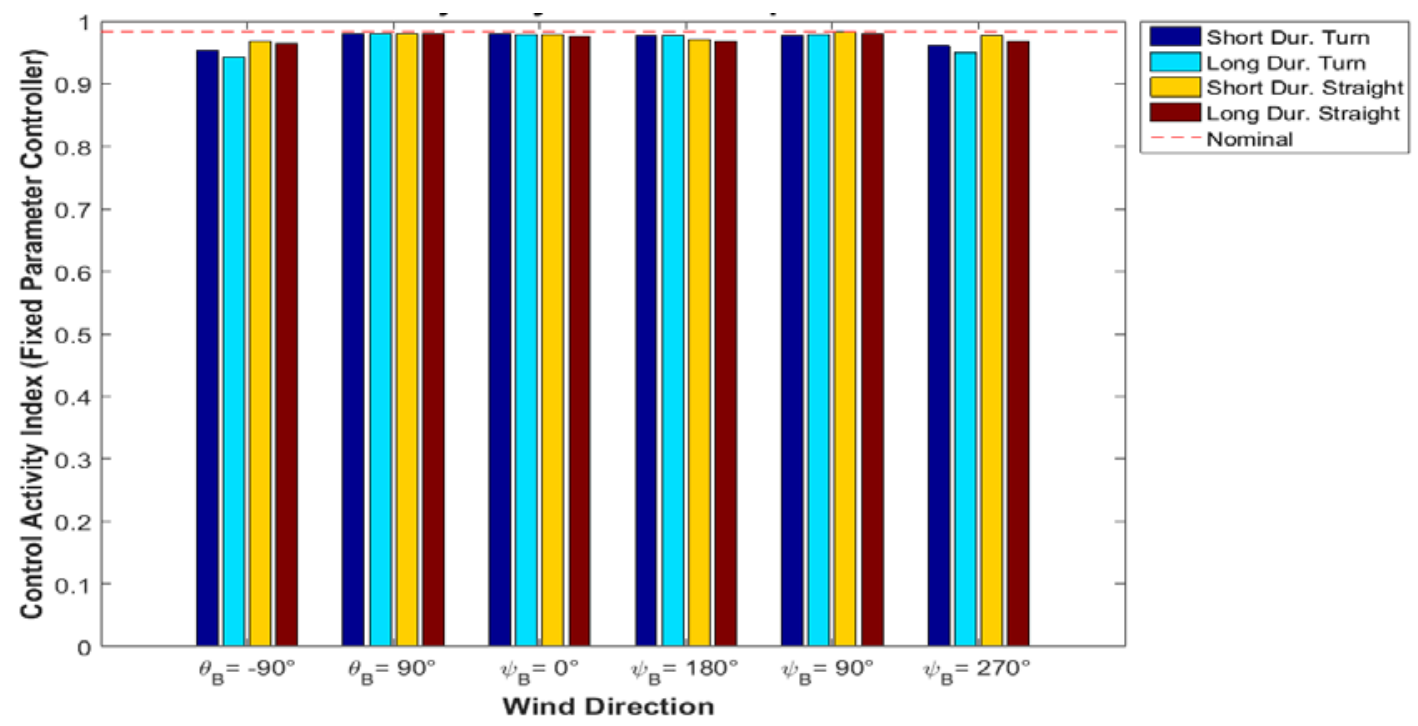

Figure 44- Activity Index for 3D S Turns Trajectory Under Low Wind Gust (Fixed PPID).

\subsection{Constant Wind and Turbulence Analysis}

As illustrated in Figure 45, it can be concluded that as the turbulence level increased, the trajectory tracking performance decreased for both controllers. This happened in the majority of the simulations for both controllers. The trajectory tracking index was also worse under strong constant wind as shown in Figure 45 and Figure 46 for both controllers. For the wind direction with downward components ( $\Psi_{\mathrm{w}}$ and negative $\theta_{\mathrm{w}}$ combined), the performance index was zero in the majority of the cases under high speed constant wind. This means that the trajectory tracking was too poor and the error exceeded the threshold or the aircraft lost control. 


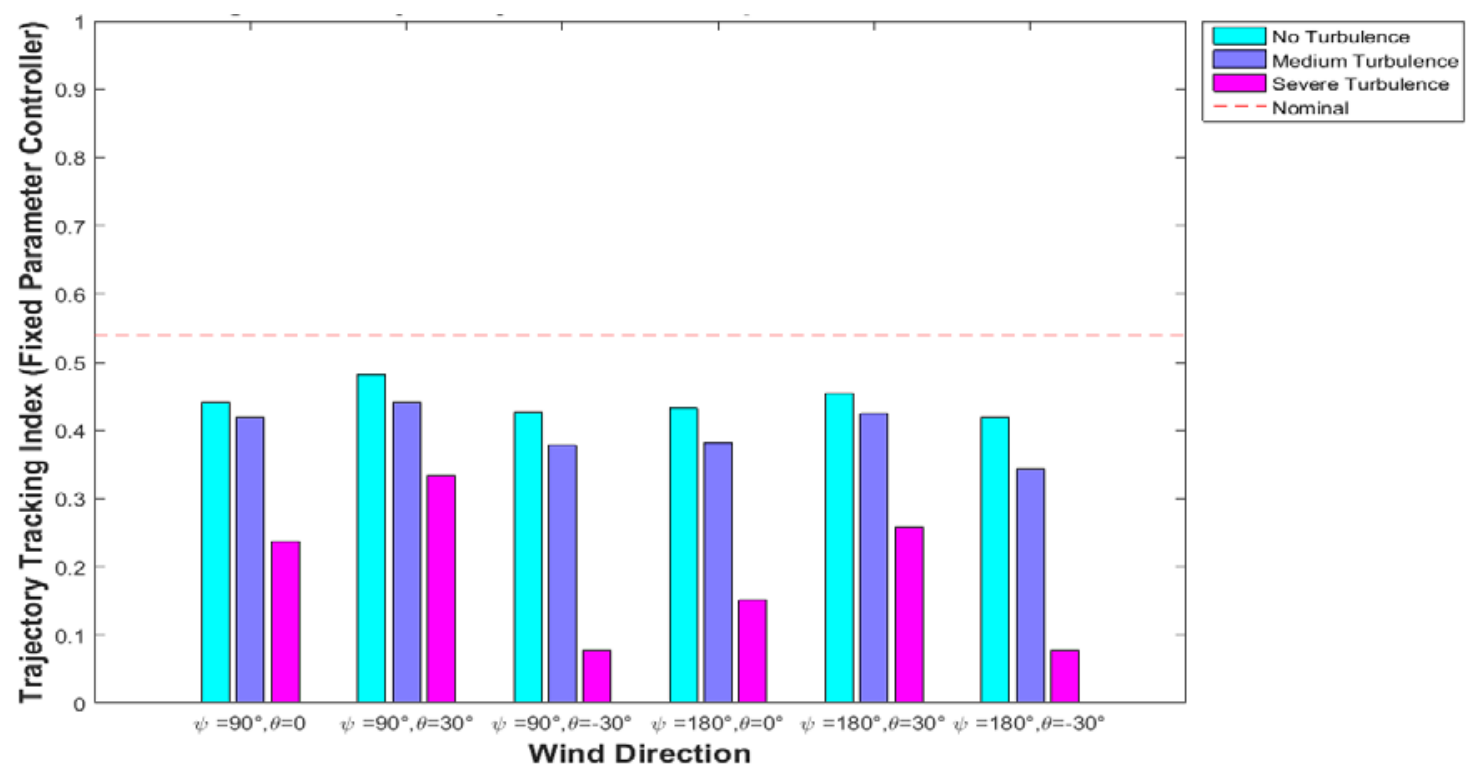

Figure 45- Tracking Index for Figure 8 Trajectory Under Low Constant Wind (Fixed PPID).

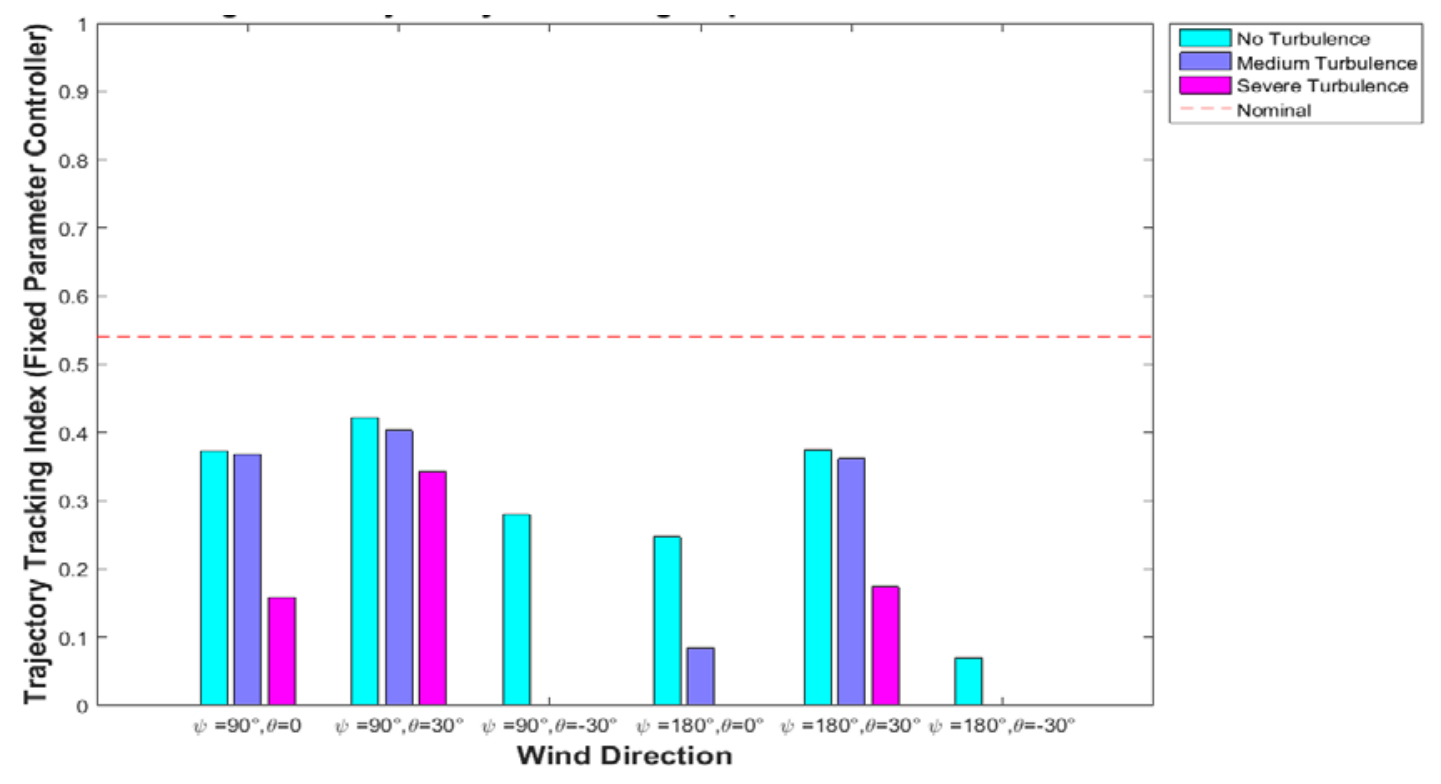

Figure 46- Tracking Index for Figure 8 Trajectory Under Strong Constant Wind (Fixed PPID).

It was observed throughout the simulation that just as the negative $\theta_{\mathrm{w}}$ (downward component) contributed to poor performance, positive $\theta_{w}$ (upward component) often resulted in higher performance indices. Such tendency can be seen in Figure 47 and Figure 48, where performance index is zero for all cases except with positive $\theta_{w}$. As compared to the case with no vertical wind component, the downdraft case reduces aircraft angle of attack, while the updraft increases it. This will result in reducing the lift and hence control moments produced by the control surfaces in the first case and increasing them in the second. Therefore, the downward 
wind will produce an equivalent decrease of control derivatives for stabilator and aileron, while the upward wind will produce an increase of the same control derivatives. As a result of the combination of aircraft and control system characteristics, the control effectiveness will be higher in the presence of upward wind and lower in the presence of downward wind. However, further testing and analysis has to be performed to confirm this preliminary explanation and establish the extent to which the observed trends can be generalized.

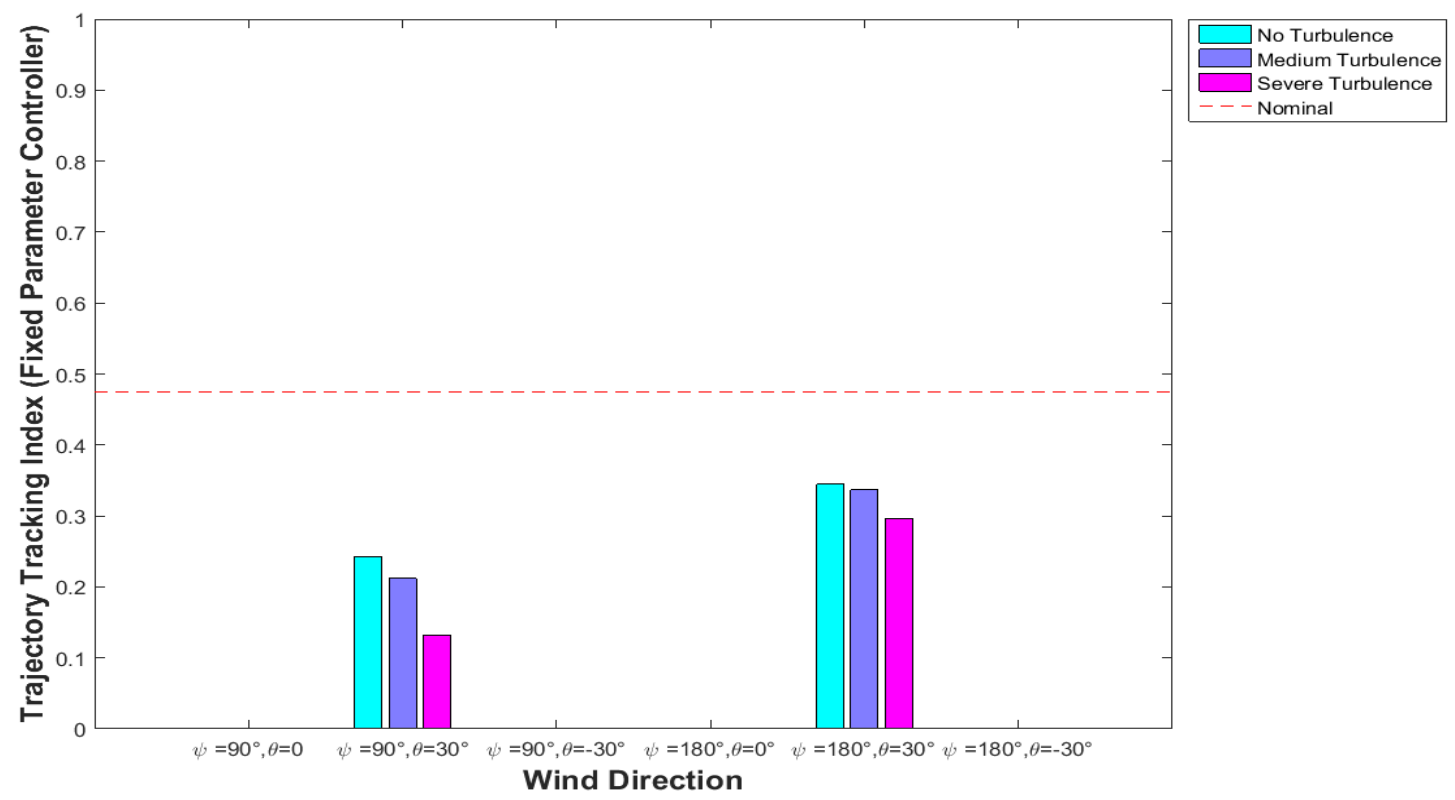

Figure 47- Tracking Index for Clothoid trajectory Under Strong Constant Wind (Fixed PPID).

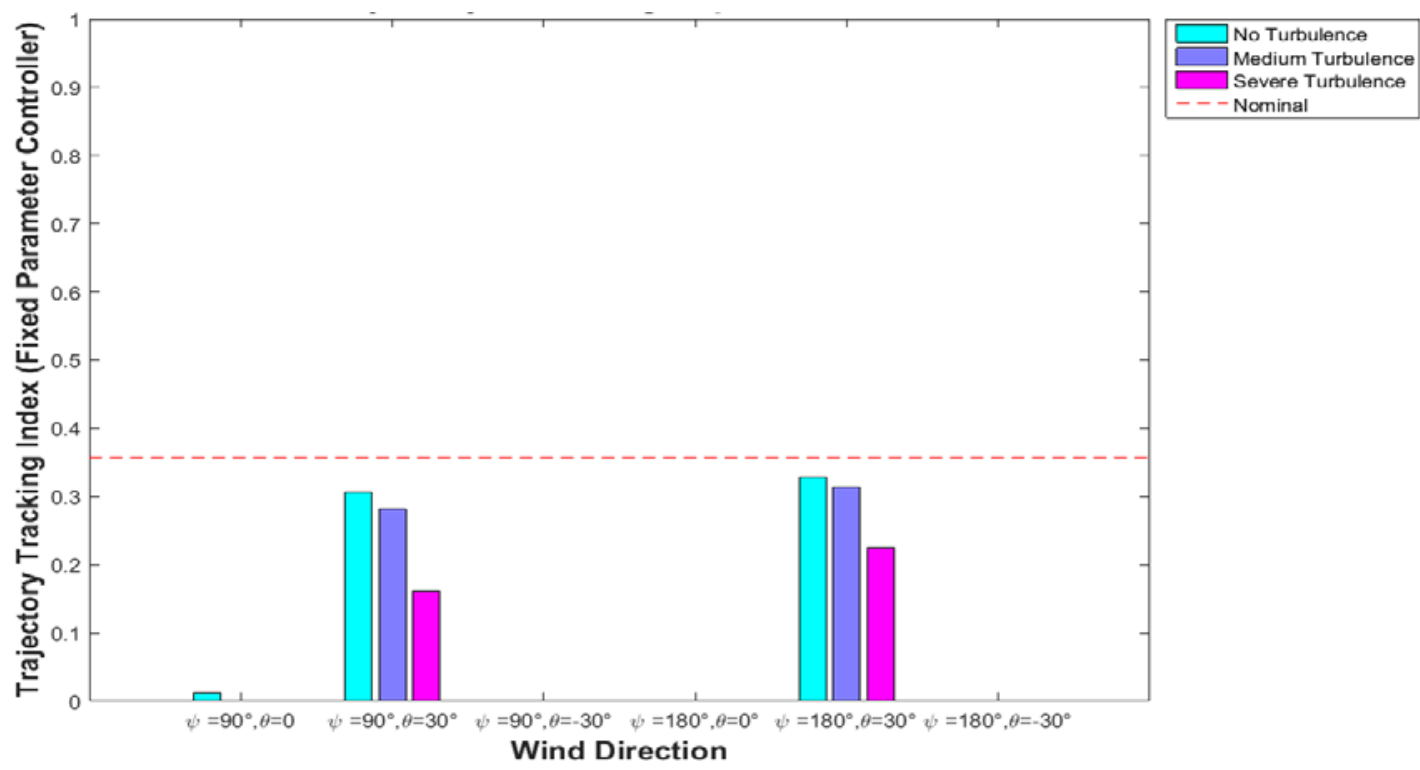

Figure 48- Tracking Index for Dubins trajectory Under Strong Constant Wind (Fixed PPID). 
For some trajectories, the performance indices were practically the same for no turbulence and medium turbulence, especially for adaptive controller under high constant wind. These results suggest a non-linear degradation of the trajectory tracking performance with turbulence severity, as illustrated in Figure 30. For fixed parameter controller under high constant wind, the effect of turbulence severity appears to be more linear, see Figure 46.

For 3D s turns, both controllers performed well for medium and no turbulence under low constant wind. The performance under wind influence is close to nominal values for both controllers (Figure 39 and Figure 49). Regarding this trajectory, a peculiar case was noted for the adaptive controller with an initial head wind $\left(\Psi_{\mathrm{w}}=180^{\circ}\right)$ in addition to positive $\theta_{\mathrm{w}}$ component and no turbulence. The controller performs better than in nominal condition, this could be due to the increase in lift generated by the wind (Figure 49). This may affect the controller since the weight for the $z$ component is higher than for xy component as mentioned before. Moreover, under severe turbulence the index is poor for almost all wind directions under strong constant wind. Similar tendency can be seen for fixed parameter controller as illustrated in Figure 50.

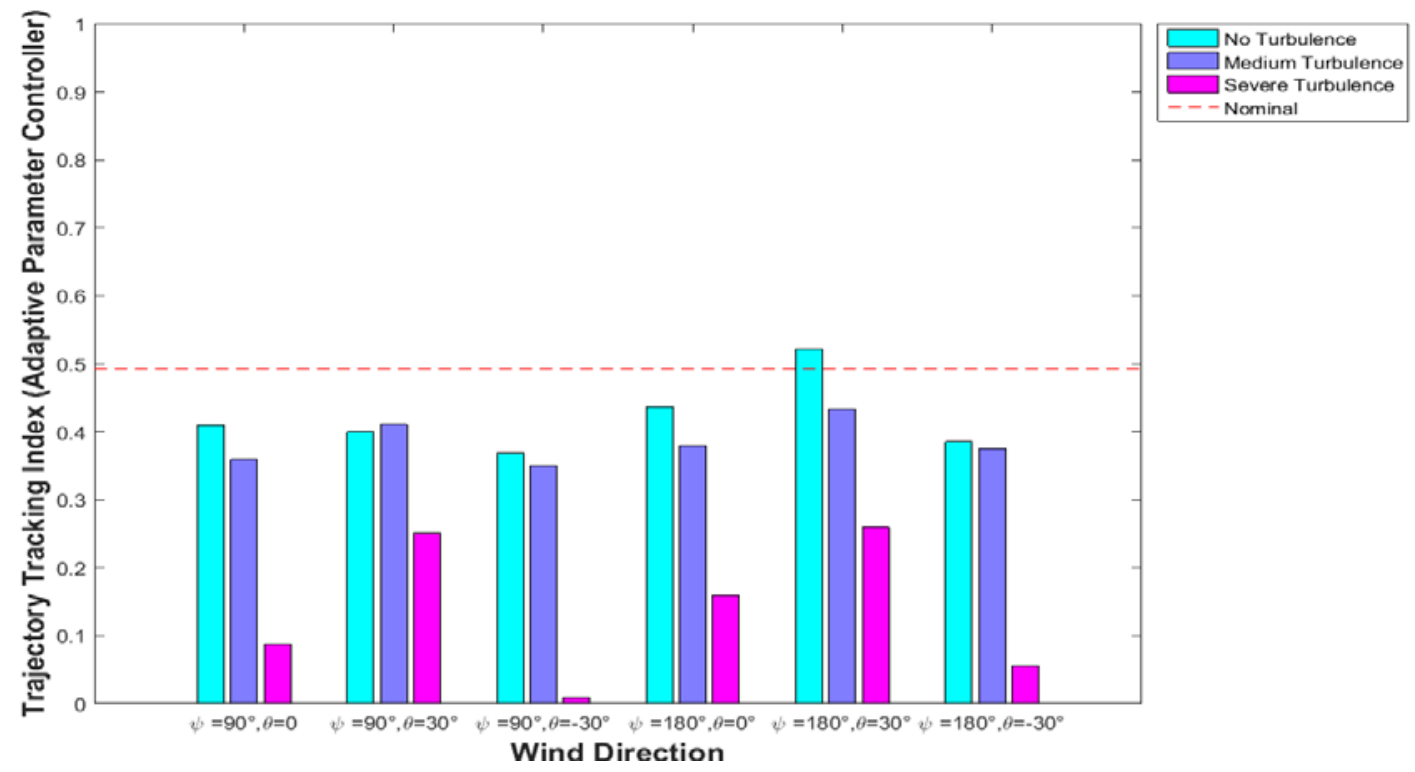

Figure 49- Tracking Index for 3D S Turns Trajectory Under Low Constant Wind (Adaptive PPID). 


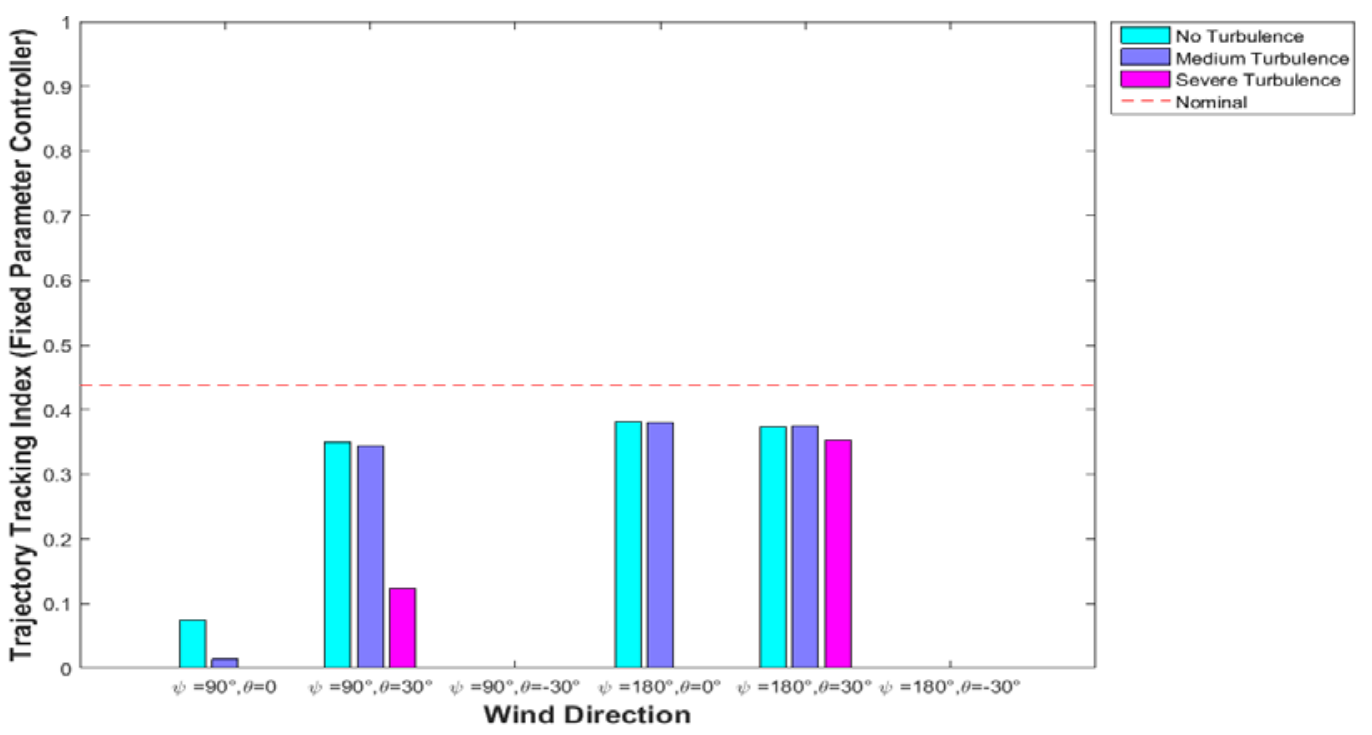

Figure 50- Tracking Index for 3D S Trajectory Under Strong Constant Wind (Fixed PPID).

The control activity increased as turbulence severity increased, as expected. Thus, the performance index values decreased. However, there was no relevant difference among wind directions or wind magnitude. This is illustrated in Figure 35.

\subsection{Wind Gust Analysis}

The aircraft fixed parameter controllers, in general, was less affected (presented smaller changes) by wind gust than the adaptive controllers, which was an unexpected trend. The performance index values are not so distant from the nominal values for majority of the cases for the fixed parameter controller. Furthermore, there was less variation among the cases for the fixed parameter than for the adaptive controller. Although, as seen for constant wind, negative values of $\theta_{\mathrm{G}}$ also resulted in loss of control for wind gusts for both controllers. Under high speed gust as a downward burst, the aircraft lost control or had a very poor performance in trajectory tracking, with errors that exceeded the thresholds which resulted in zero index value. This happened for all trajectories for $\theta_{B}=-90^{\circ}$ (with exception of 3D s turns) as shown in Figure 51 and Figure 52. Under short duration gust, both controllers presented better response during straight flight segment path than in turns. Exceptions happened for tail wind or wind initially blowing upwards $\left(\Psi_{B}=0^{\circ}\right.$ and $\theta_{B}=90^{\circ}$ respectively), which could be due to the tail wind becoming lateral wind as the aircraft follows the trajectory, this may facilitate the aircraft to turn. A possible explanation for the upward gust trend is that, since the aircraft is tilted during the turn, a short gust may not affect the aircraft flight as much as it would if the aircraft was flying in a straight path. 


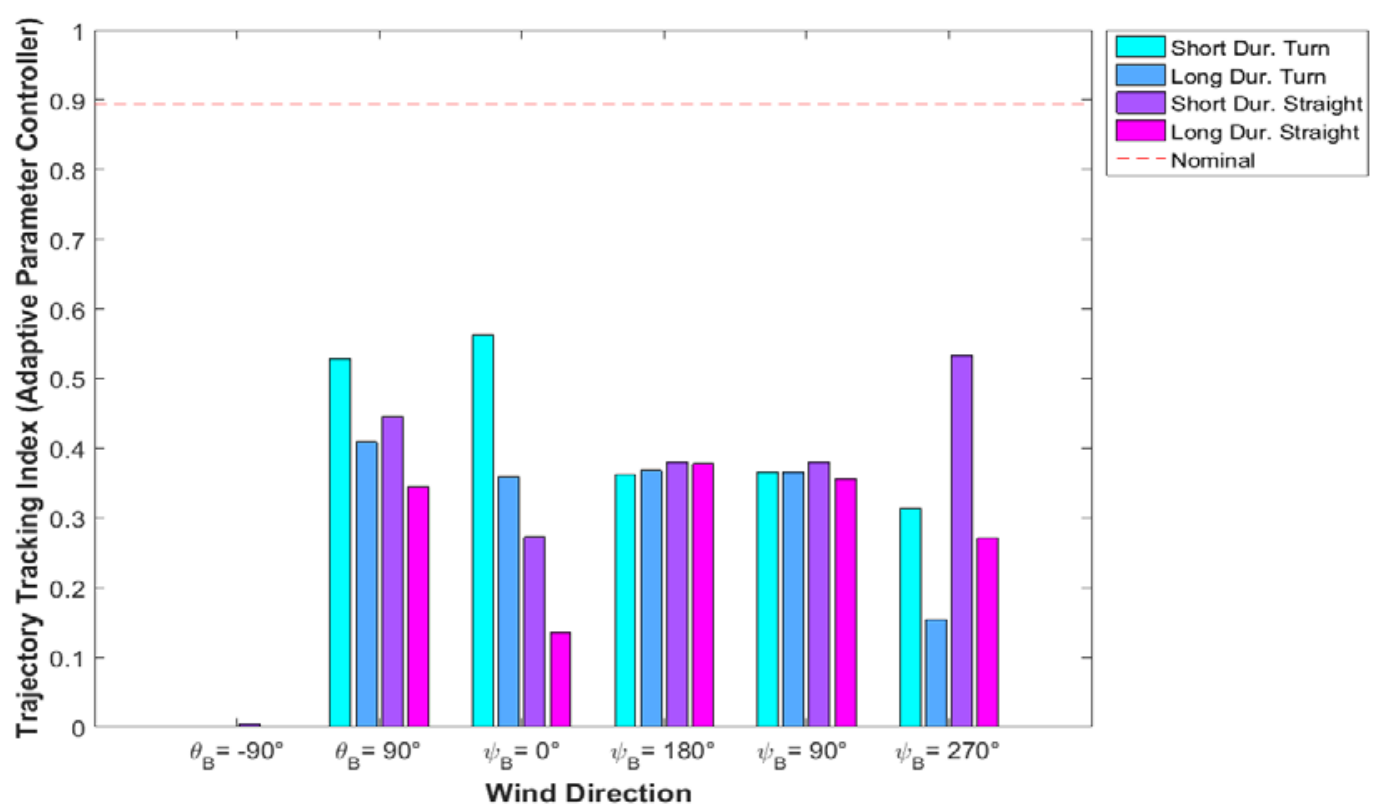

Figure 51- Tracking Index for Figure 8 Trajectory Under Strong Wind Gust (Adaptive PPID).

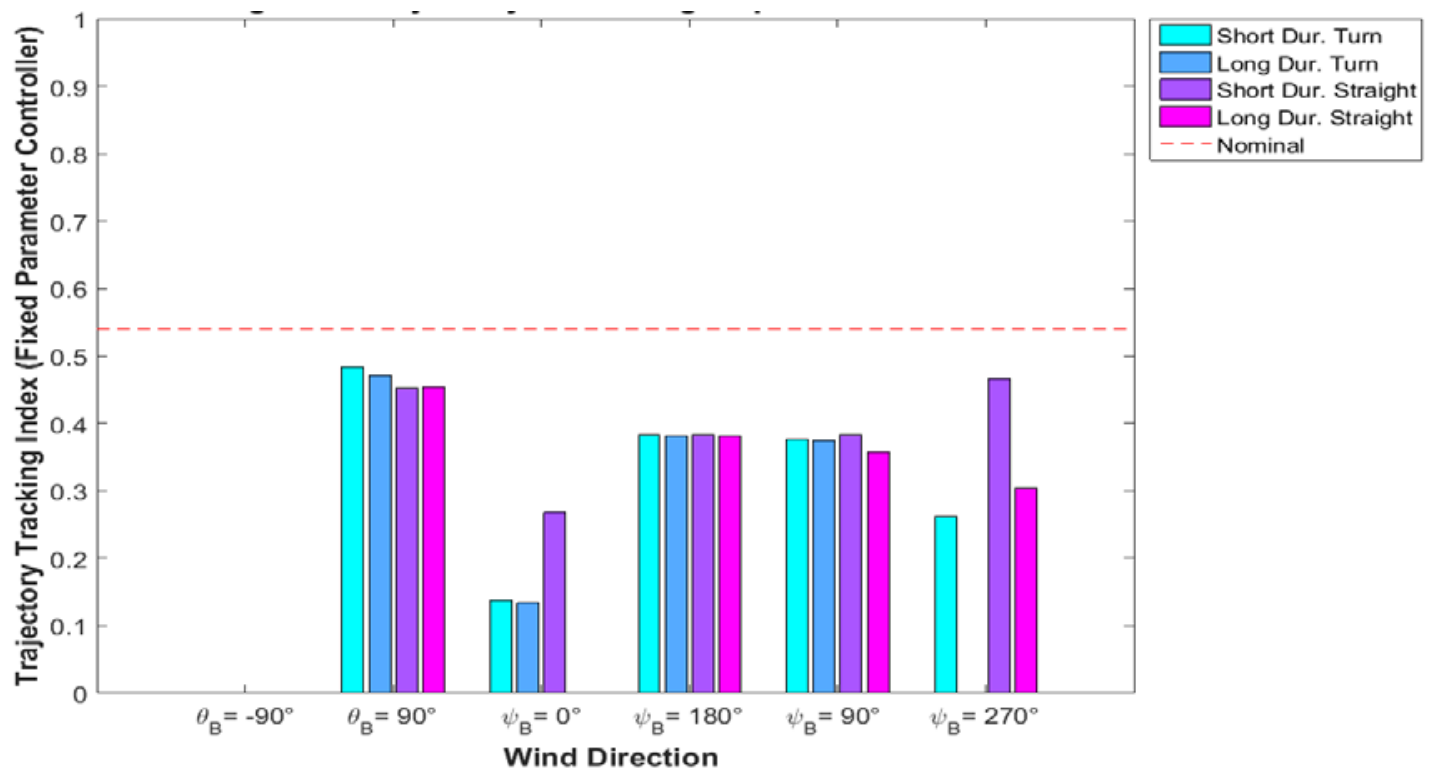

Figure 52- Tracking Index for Figure 8 Trajectory Under Strong Wind Gust (Fixed PPID).

There were small variation between long and short duration gusts, for the fixed parameter controller in all trajectories, for the majority of the cases (Figure 52 and Figure 53). On the other hand, the adaptive controller appeared to be more sensitive (higher variation) regarding gust duration. The index values were higher for short duration than for long duration gust (Figure 54, Figure 55 and Figure 58). 


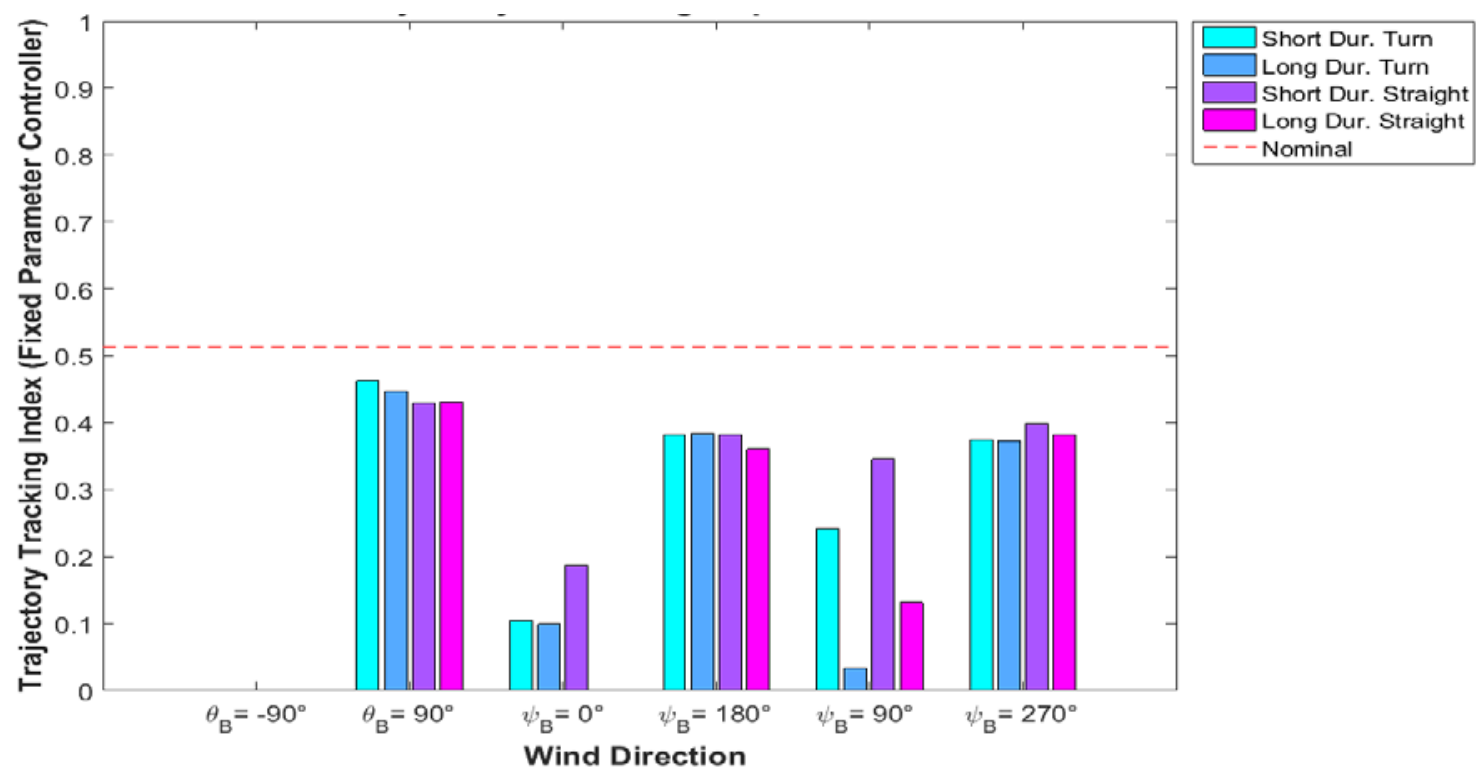

Figure 53- Tracking Index for Oval Trajectory Under Strong Wind Gust (Fixed PPID).

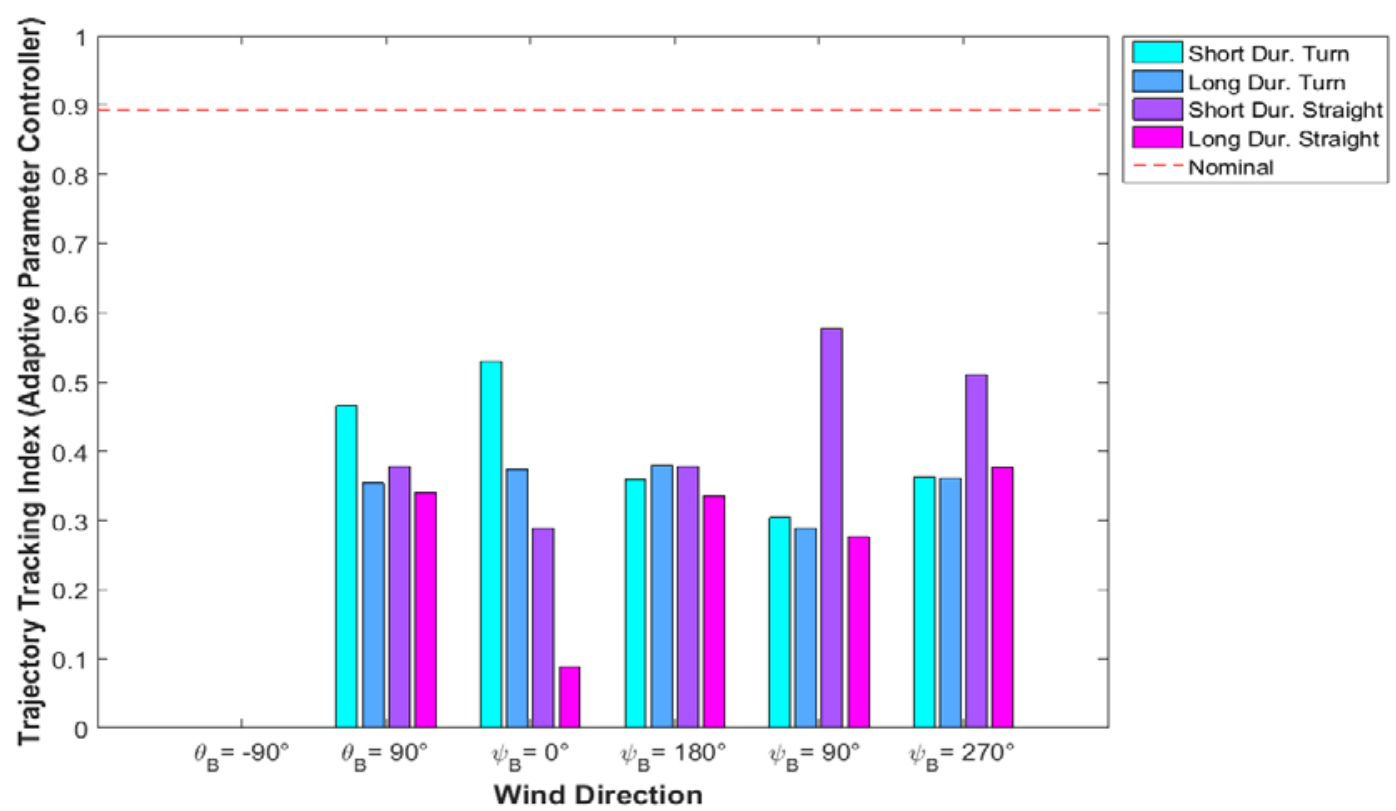

Figure 54- Tracking Index for Oval Trajectory Under Strong Wind Gust (Adaptive PPID).

For the 3D s turns trajectory, the wind gusts appeared to be less troublesome than for other trajectories. This was consistent with the trend noticed for constant wind. The performance index values are closer to nominal than for other trajectories and variations among the cases are smaller (Figure 54 and Figure 55). 


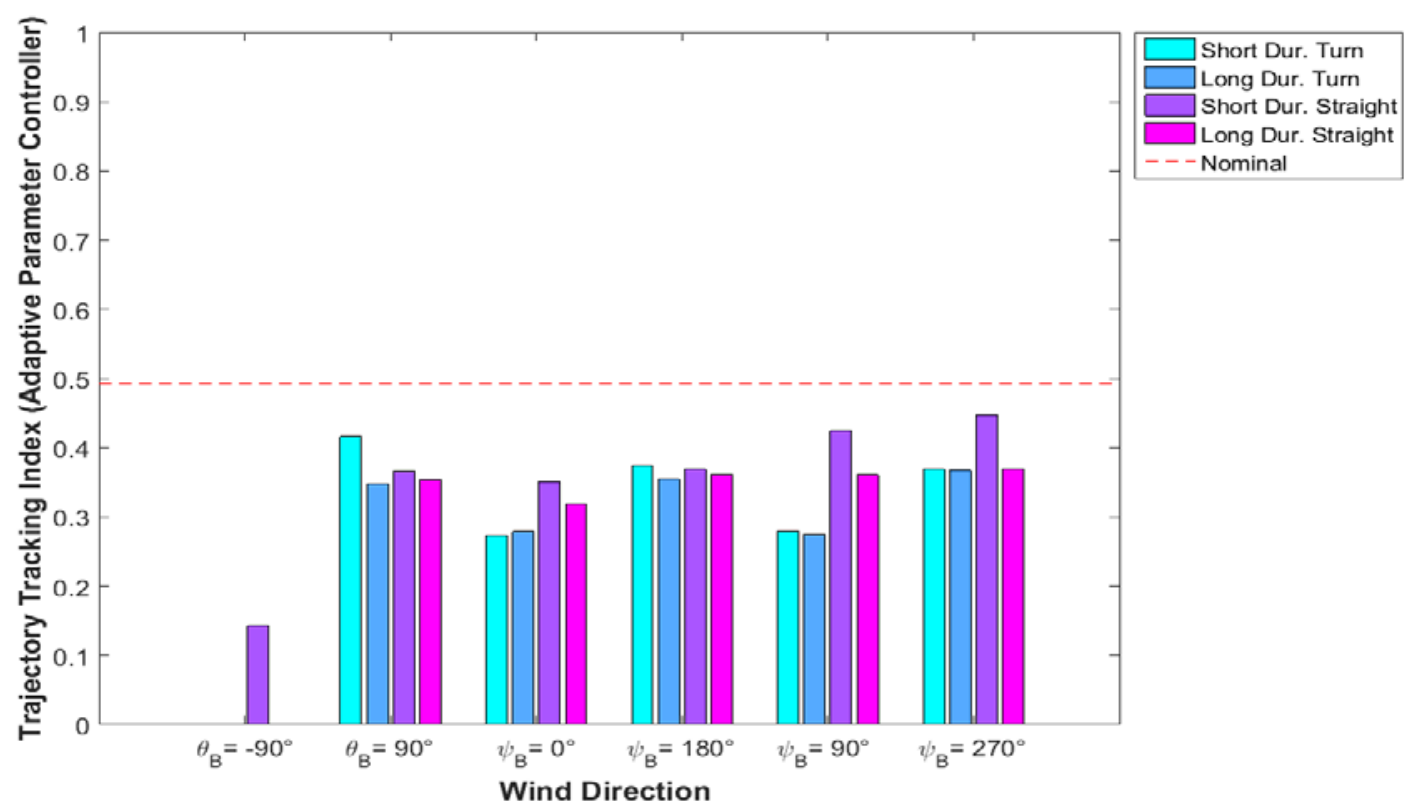

Figure 55- Tracking Index for 3D S Turns trajectory Under Strong Wind Gust (Adaptive PPID).

The fixed parameter controller under low speed gust presented good performance index for all cases for all trajectories. The majority of the values reach and slightly exceeded the index value for nominal conditions (Figure 56), or stay close to nominal values (Figure 57). Even for downburst, both controllers present higher performance indices than under strong wind gust. Under same conditions, the adaptive controller achieves better values of performance indices (Figure 57 and Figure 58). However, the relative deterioration of performance exhibited by the adaptive control laws is, in general, larger than the one of the fixed parameter controller.

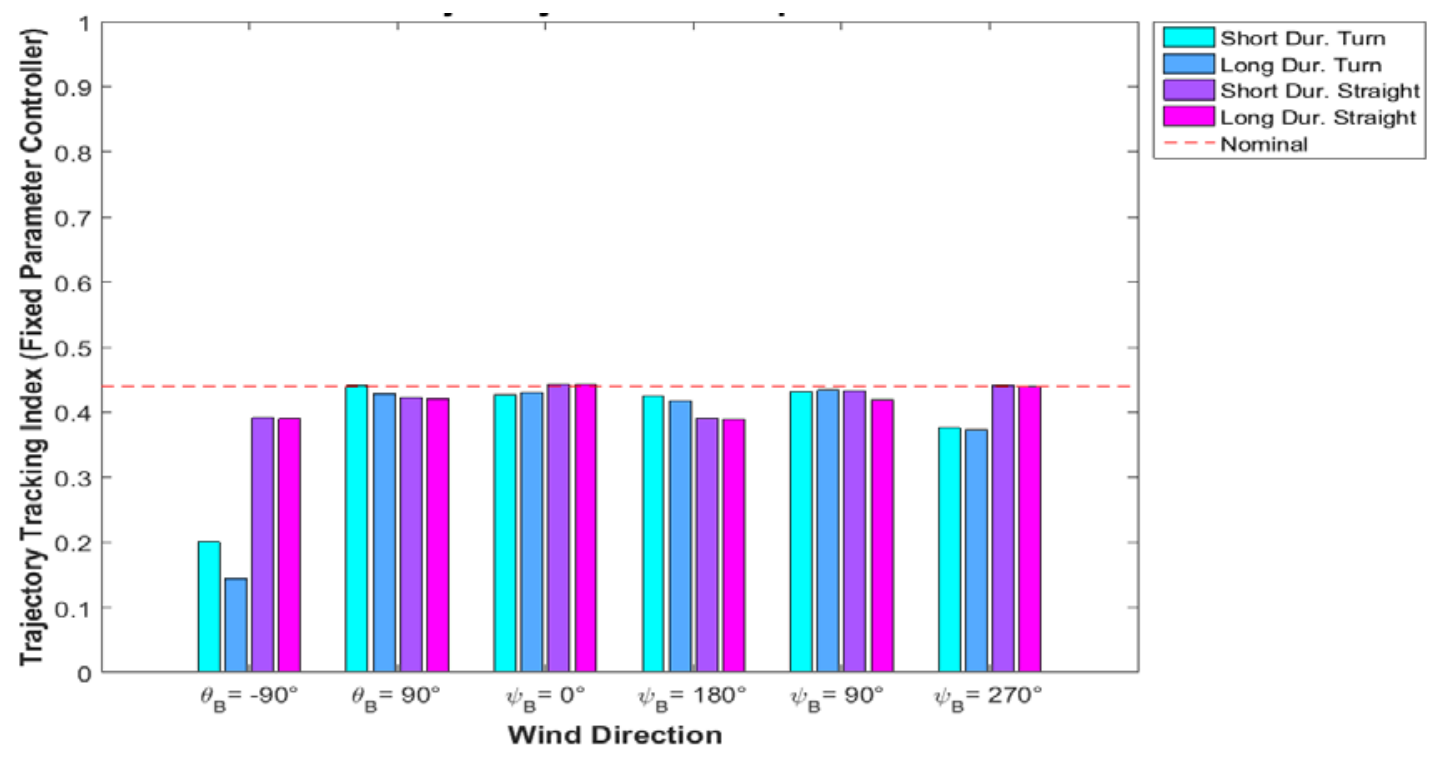

Figure 56- Tracking Index for 3D S Turns trajectory Under Low Wind Gust (Fixed PPID). 


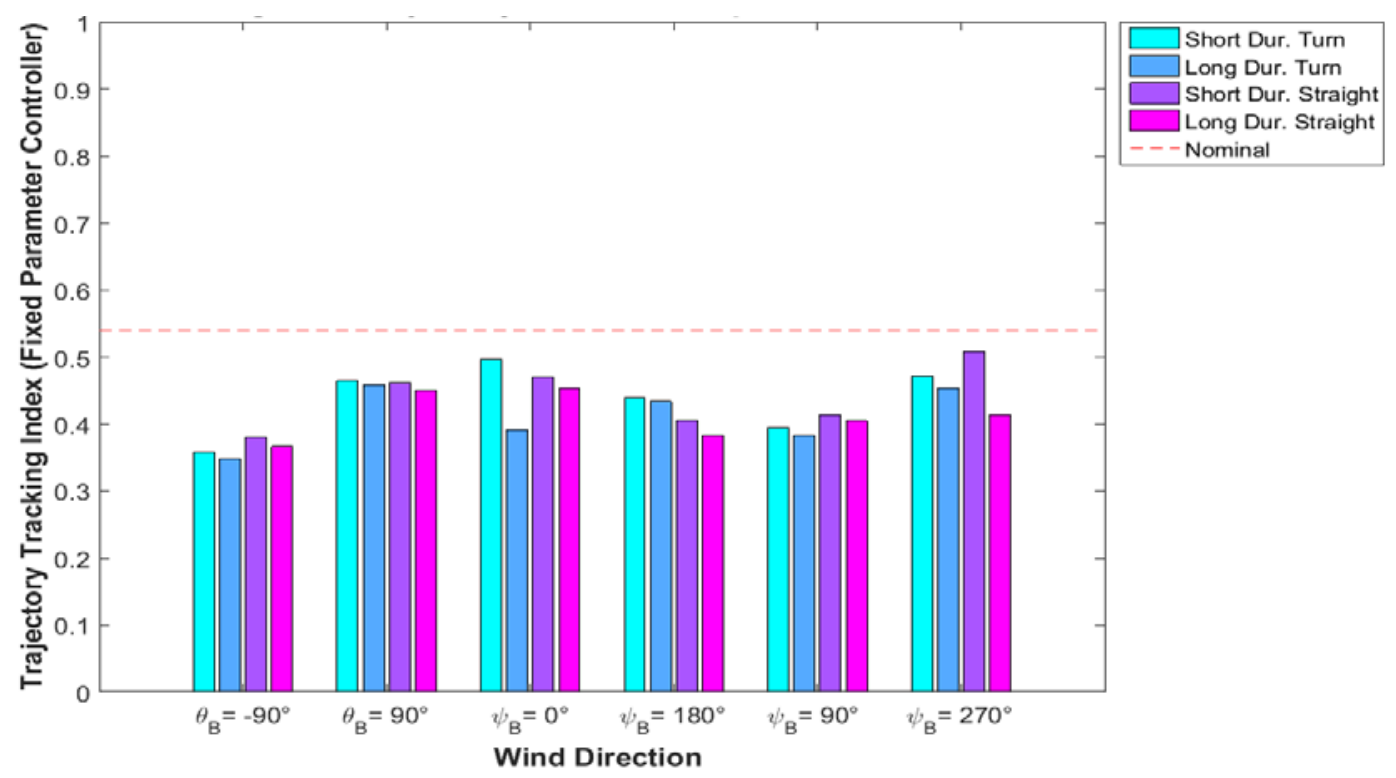

Figure 57- Tracking Index for Figure 8 trajectory Under Low Wind Gust (Fixed PPID).

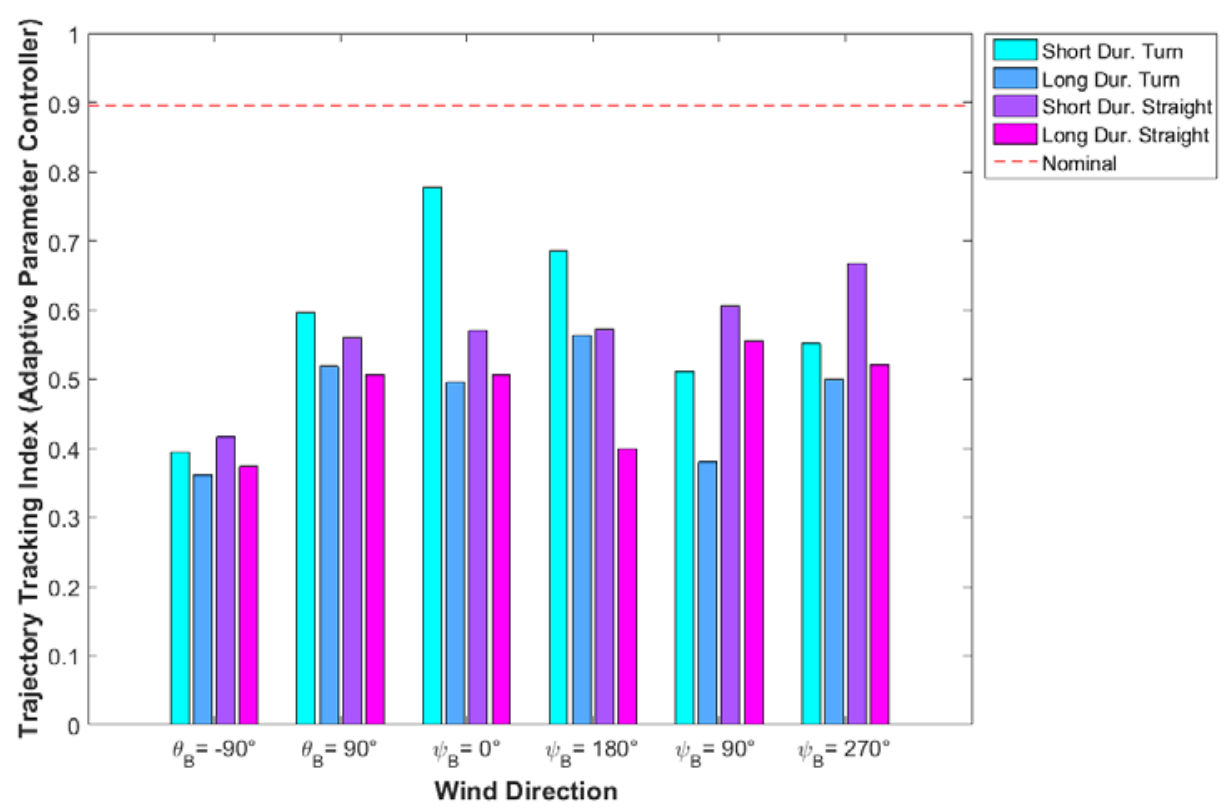

Figure 58- Tracking Index for Figure 8 trajectory Under Low Wind Gust (Adaptive PPID).

The control activity did not change much as magnitude was increased or for different trajectories. The performance indexes were high and close to nominal values, although, the lowest index was found for $\theta_{B}=-90$ for all trajectories. This is illustrated in Figure 43.

\subsection{Wind Shear Analysis}

For low wind shear, the performance index was lowest for vector component along the $X$ axis, and the gradient direction along $\mathrm{X}$. Moreover, zero performance index was obtained for 
strong wind shear. For straight flight segment, both controllers presented a better response than for turn flight segment in most cases, which is consistent with the turn being a slightly more difficult maneuver. Regarding the $Y$ vector component, the lowest performance obtained was with respect to gradient direction along the $Y$ axis. The $Z$ vector component presented basically the same index value independent of gradient direction or location of the wind shear (turn or straight flight) for oval and figure 8 trajectory. When the magnitude of the wind shear was increased, the trends were practically the same. The tracking performance index of the $Z$ component was lower than for vector components along the $X$ and $Y$ axes, since previous trends showed variation of downward wind to be so important. The trend observed is shown in Figure 59 and Figure 60.

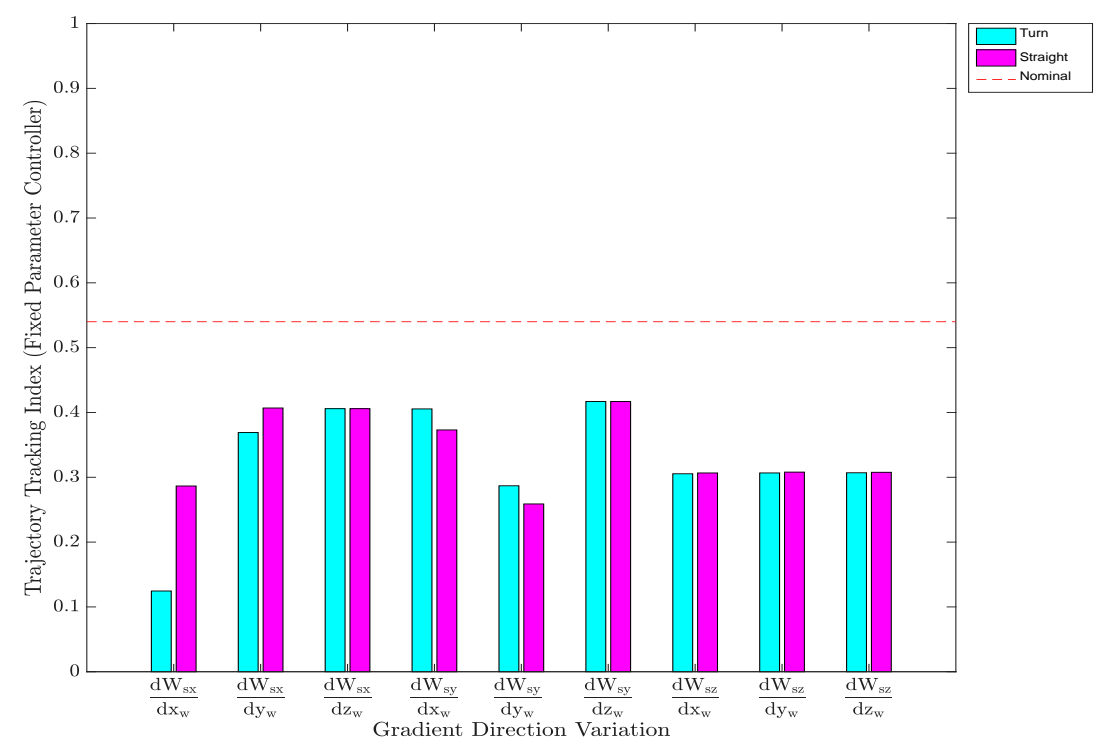

Figure 59- Tracking Index for Figure 8 Under Low Wind Shear (Fixed PPID). 


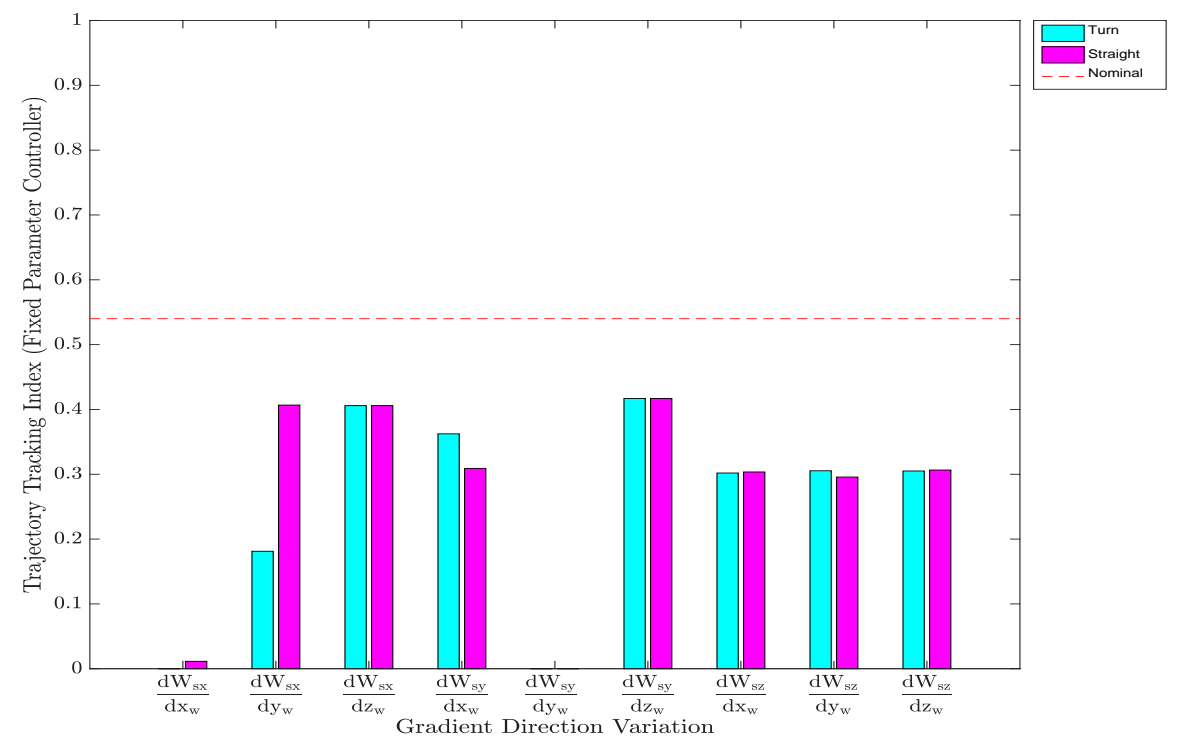

Figure 60-Tracking Index for Figure 8 Under Strong Wind Shear (Fixed PPID).

There were very small differences between the adaptive and fixed PPID controllers under same wind shear conditions. The vector component along the $Z$ axis still followed previous trend, where index values were practically the same for any variation of the gradient direction (Figure 61 and

Figure 62).

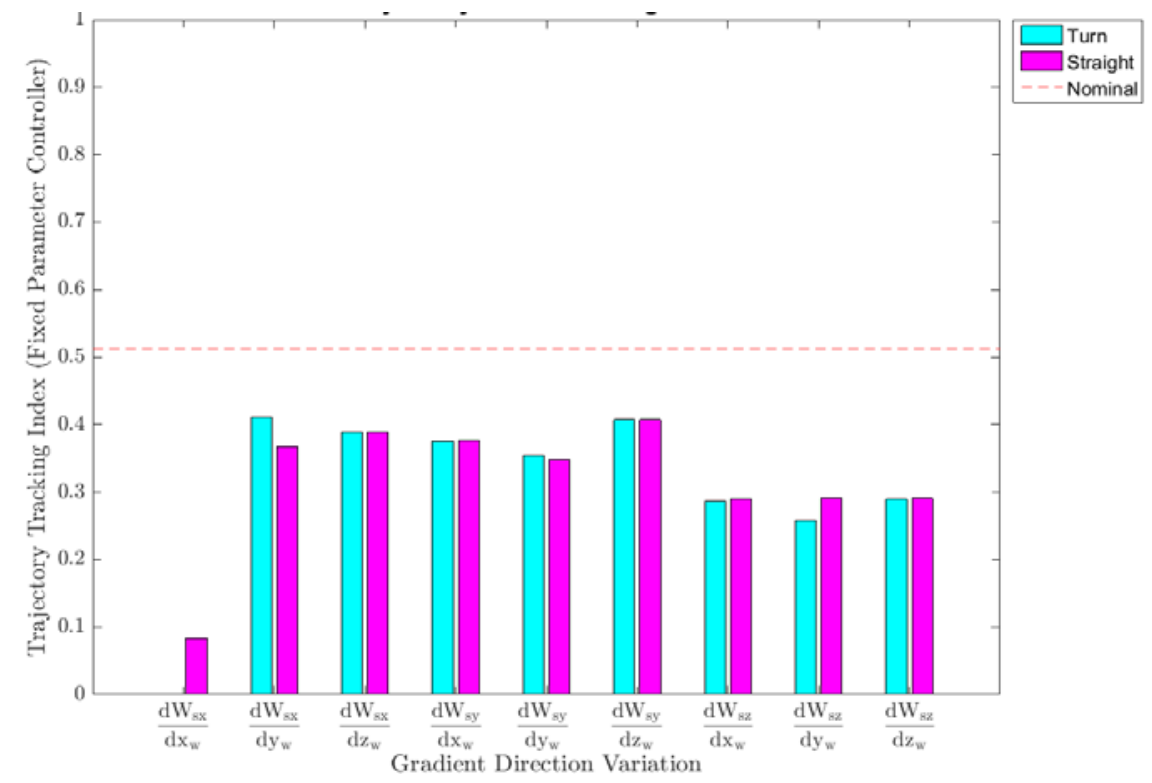

Figure 61- Tracking Index for Oval Trajectory Under Strong Wind Shear (Fixed PPID). 


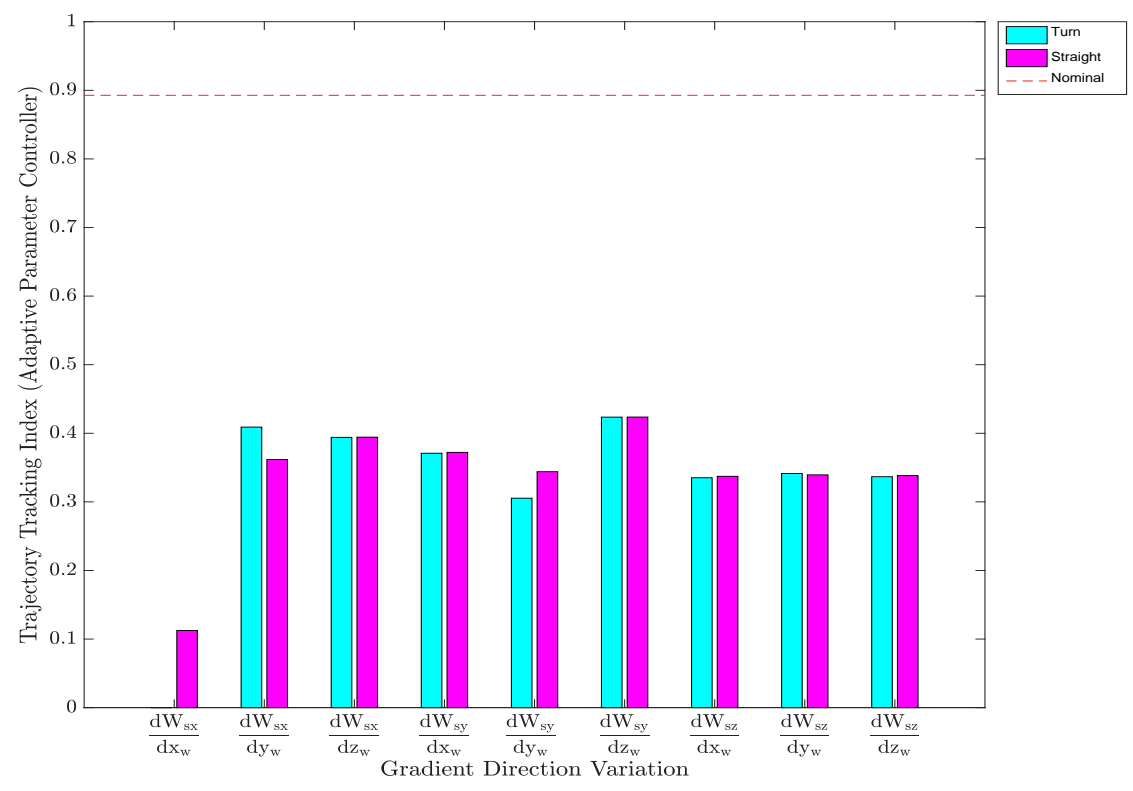

Figure 62- Tracking Index for Oval Trajectory Under Strong Wind Shear (Adaptive PPID).

A smoother performance index variation was obtained for 3D s turns trajectory, than for other trajectories for all vector components. The vector component along the $Z$ axis should only vary for this trajectory since is the only one with significant variation in altitude (Figure 62, Figure 63 and Figure 64).

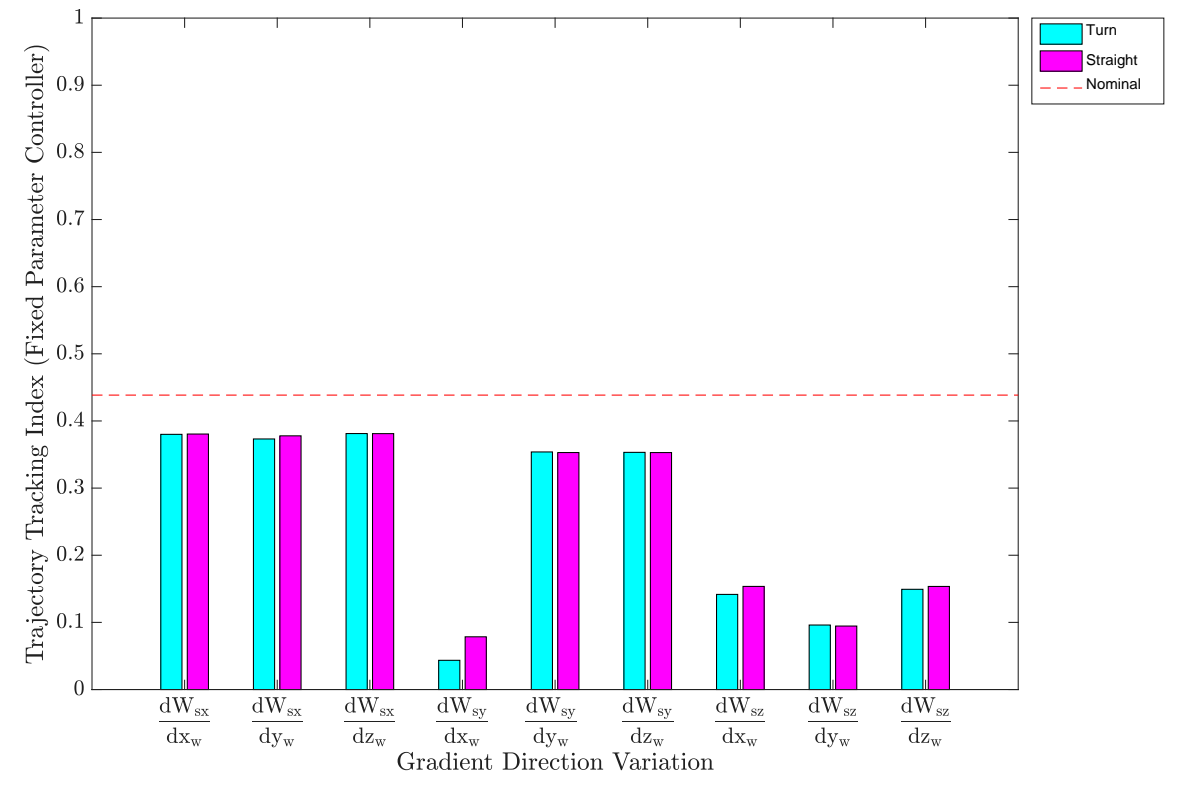

Figure 63- Tracking Index for 3D S Turns trajectory Under Strong Wind Shear (Fixed PPID). 


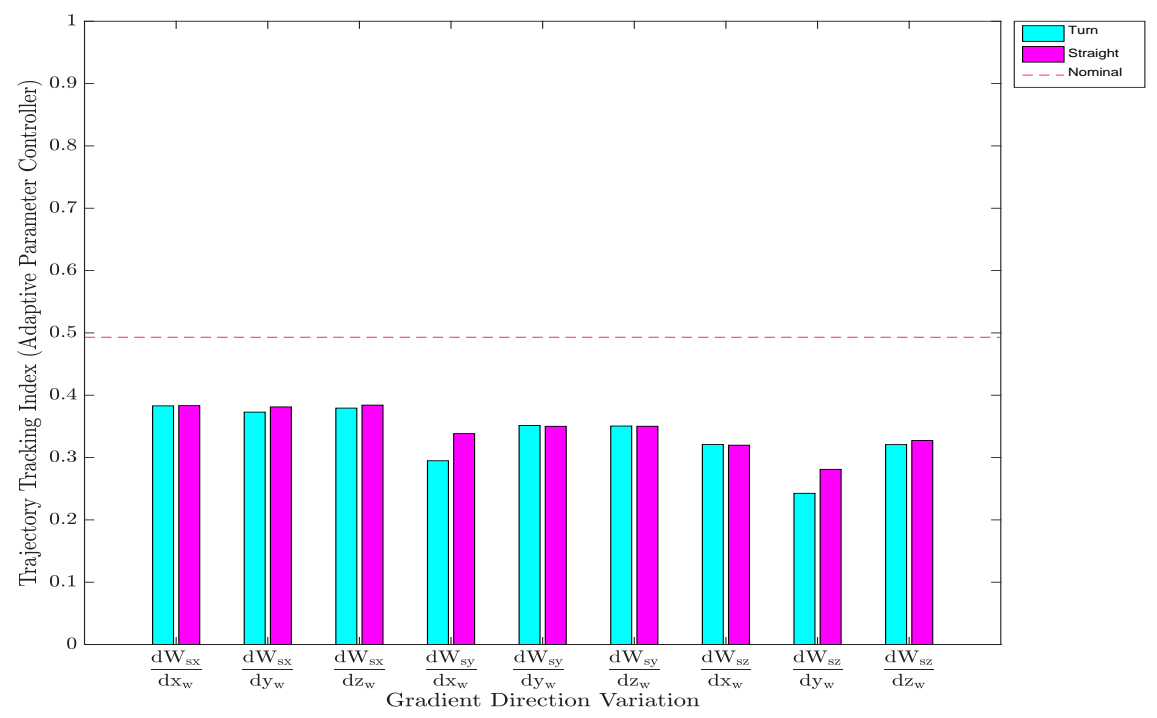

Figure 64- Tracking Index for 3D S Turns trajectory Under Strong Wind Shear (Adaptive PPID).

Overall, the fixed parameter controller seemed to have a resilience to wind shear when compared to adaptive controller. The performance index values are closer to nominal conditions for the fixed parameter controller than for the adaptive controller, meaning that relative degradation of performance for the adaptive control is larger. However, even with nominal performance index value farther from performance indexes values obtained under wind shear, the adaptive controller presented slightly better performance indices than the fixed parameter controller. This comparison can be seen in Figure 65 and Figure 66.

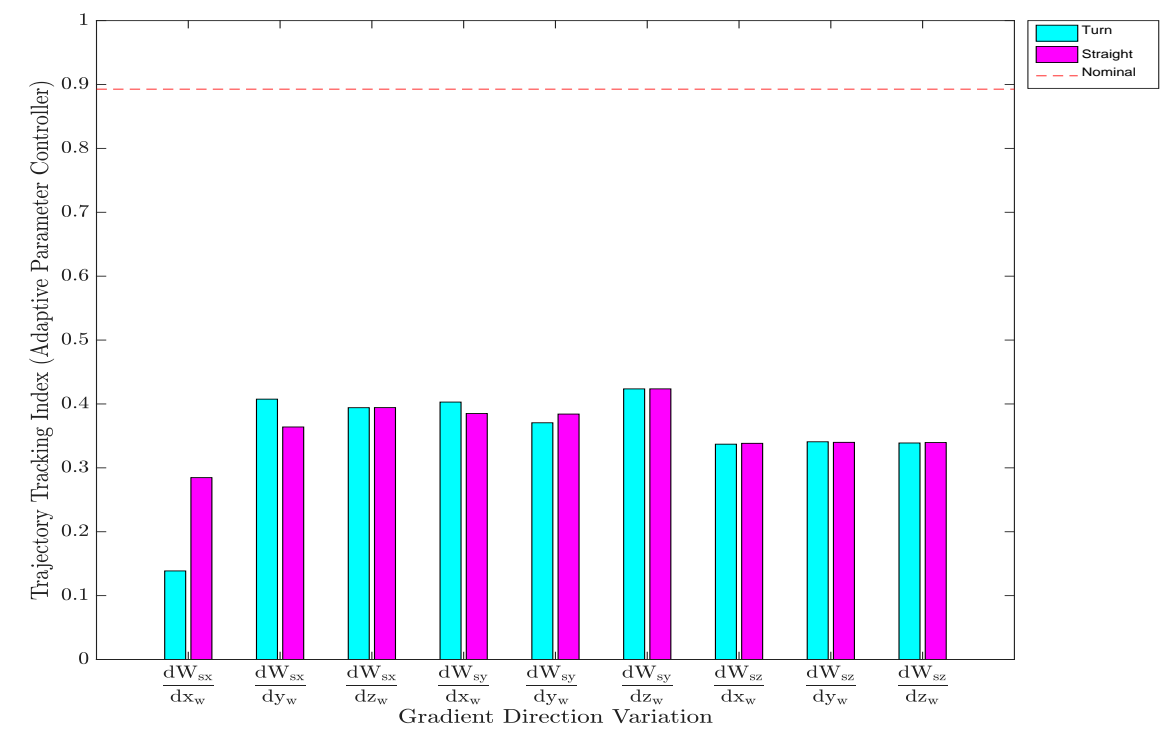

Figure 65- Tracking Index for Oval Trajectory Under Low Wind Shear (Adaptive PPID). 


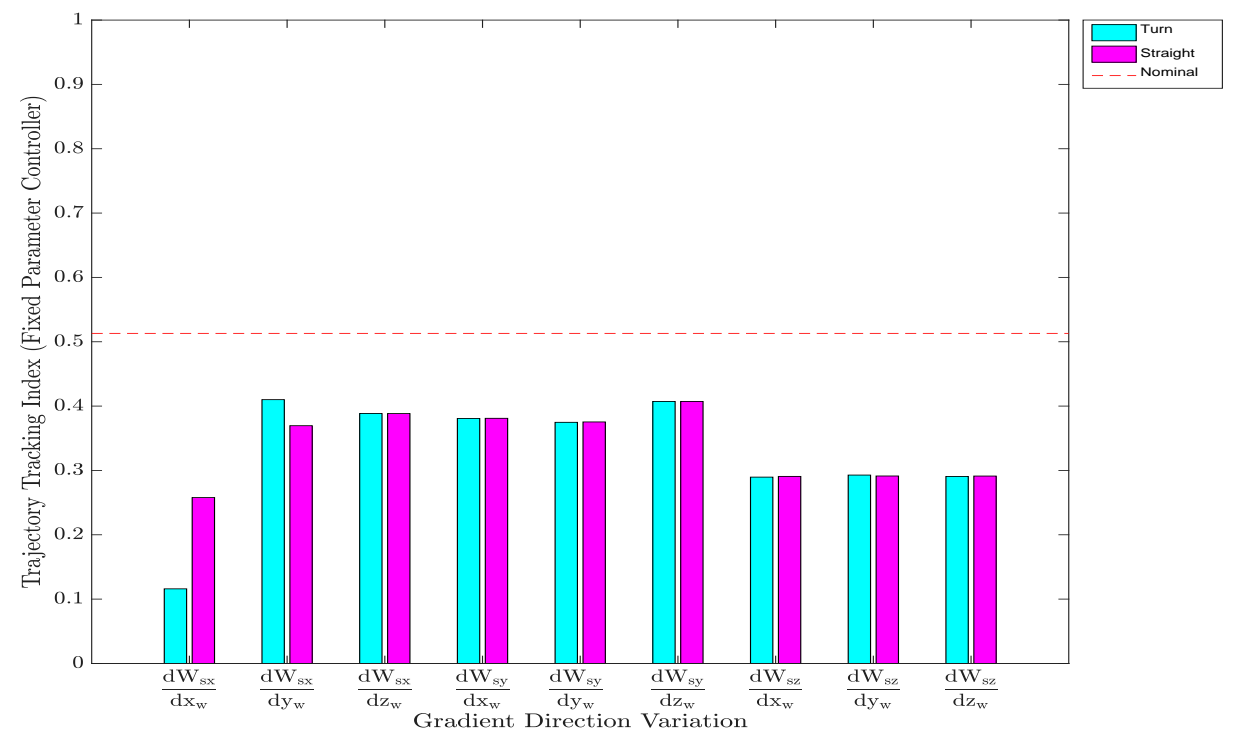

Figure 66- Tracking Index for Oval Trajectory Under Low Wind Shear (Fixed PPID).

The control activity increases for the vector component along the $Z$ axis, thus the performance indices are the lowest encountered for all cases of wind shear. For the vector component along the $\mathrm{Y}$ axis, the lowest performance index value was found for the gradient along $Y$ axis. Lastly, for vector component along the $X$ axis, the lowest performance index was found for a gradient along the $X$ axis. For different trajectories and wind shear magnitudes, there was not much difference encountered among them for control activity.

\subsection{Wind Phenomena With Actuator Failures}

Under wind gust, there was not much difference for trajectory performance index between failures in the left or right aileron. The adaptive parameter controller presented a higher performance index than the fixed parameter controller, as expected. However, both controllers reached and slightly passed the reference line for performance index, obtained where there was only gust occurring in that direction without any failure. Therefore, both controllers appeared indifferent under a medium level aileron failure. Figure 67 illustrates this discussion. 


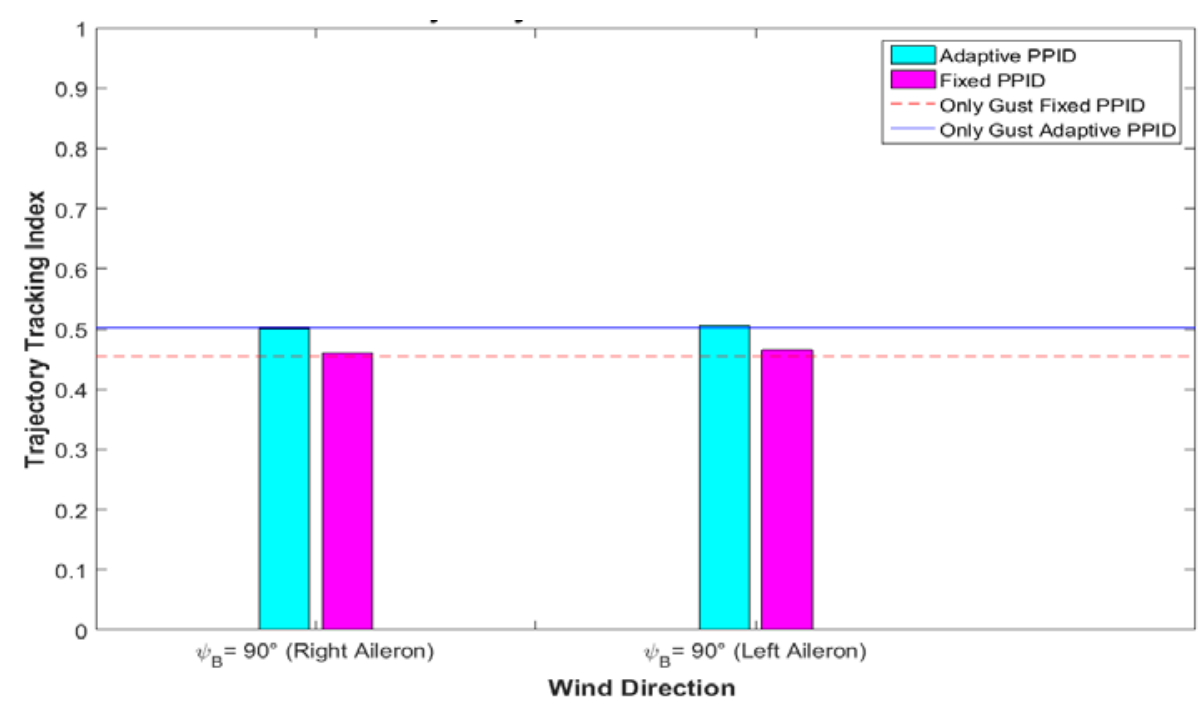

Figure 67- Tracking Index for Oval trajectory Under Wind Gust and Aileron Failure.

The same trend was noticed for control activity. The performance index is high and practically matches the same performance index values obtained under wind gust without any failure. This is shown in Figure 68.

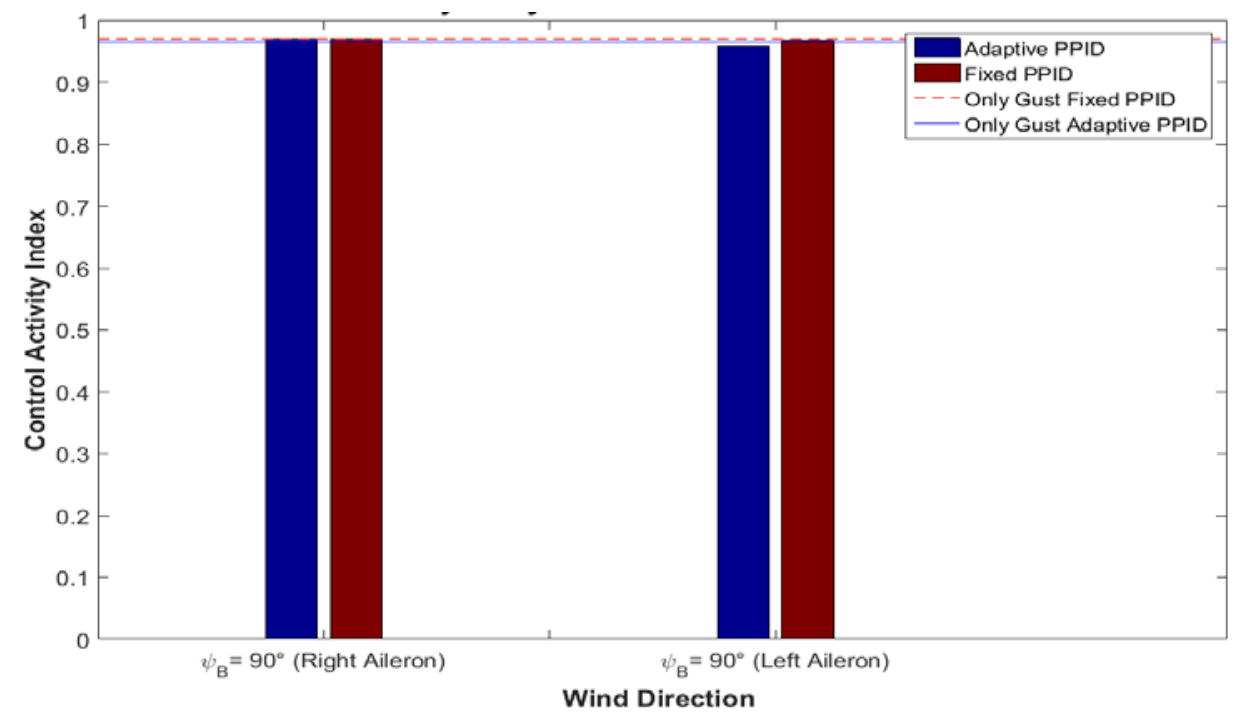

Figure 68-Control Activity for Oval trajectory Under Wind Gust and Aileron Failure.

Under constant wind influence, the failure in different position of the ailerons did not lead to any difference in the performance index values for the adaptive controller. Neither direction of the constant wind seemed to have effect on the controllers. The performance index values were the same for both directions under left or right aileron failures. Although, the performance index values under failures are lower than the values obtained while the aircraft was under constant wind only (Figure 69). Moreover, the performance index values obtained under aileron 
failures for constant wind direction of $\Psi_{\mathrm{w}}=180^{\circ}$, are closer to the reference line for constant wind only at $\Psi_{\mathrm{w}}=180^{\circ}$. Thus, it can be concluded that the adaptive controller under aileron failure performs better under constant wind at $\Psi_{\mathrm{w}}=180^{\circ}$ than at $\Psi_{\mathrm{w}}=90^{\circ}$.

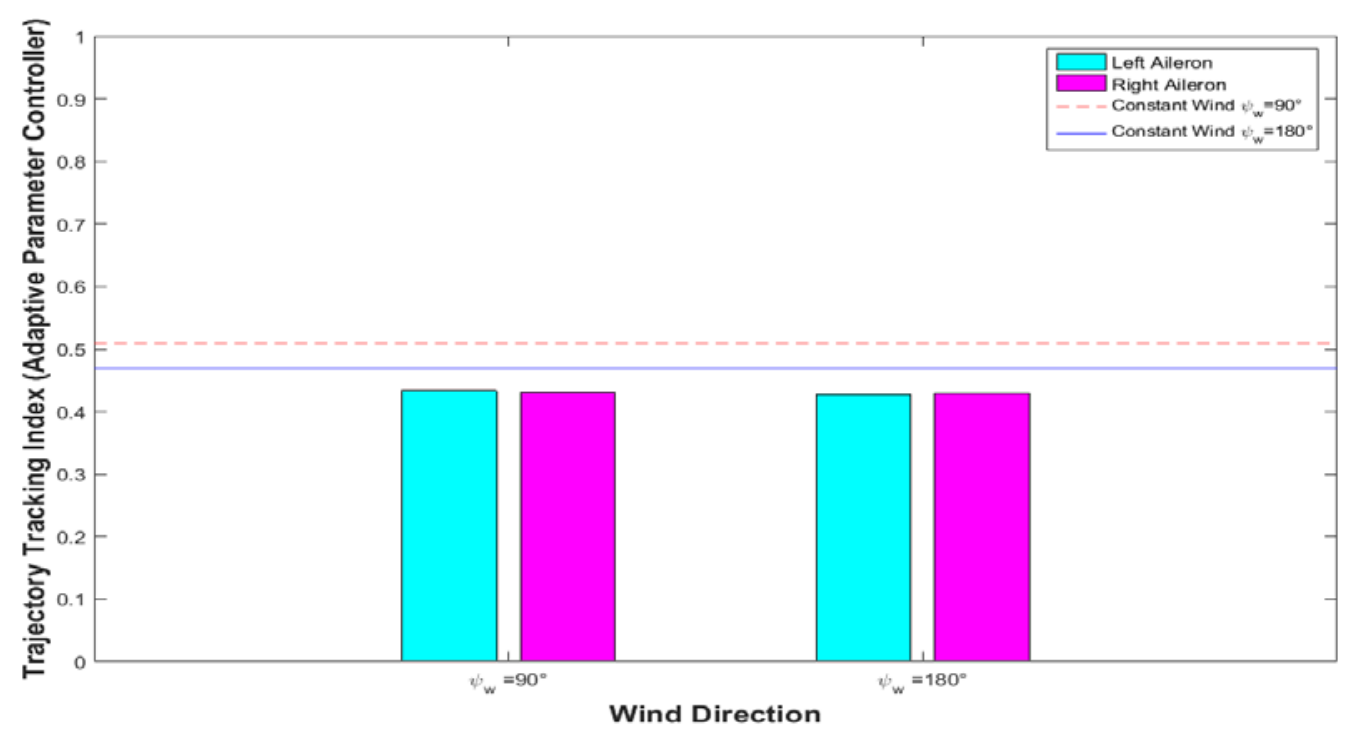

Figure 69- Tracking Index for Under Constant Wind and Aileron Failure (Adaptive PPID).

For the fixed parameter controller, the performance index values exceeded the reference line for left and right aileron failures for both wind directions. However, the controller appeared to perform better under failures in the left aileron than in the right aileron. This could be due to the flight path where the aircraft is turning right, thus, left wind and headwind will create more lift on the failed left side of the wing than on the right side. This could facilitate for the aircraft to turn right and increase the performance index. This can be seen on Figure 70.

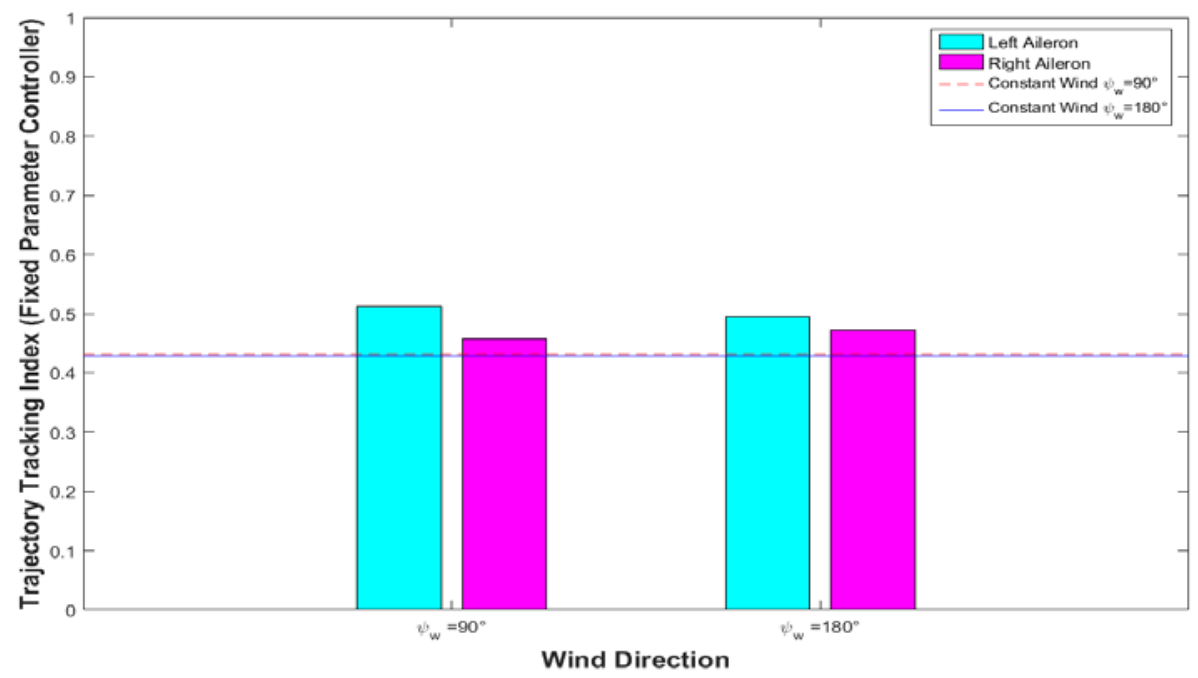

Figure 70- Tracking Index for Under Constant Wind and Aileron Failure (Fixed PPID). 
The same trend observed for aileron failures under wind gust was noted for constant wind phenomenon with aileron failures. The performance indices are almost the same for either right or left aileron failure, with the adaptive controller presenting slightly higher indices than the fixed parameter controller. Figure 71 and Figure 72 illustrate this assumption.

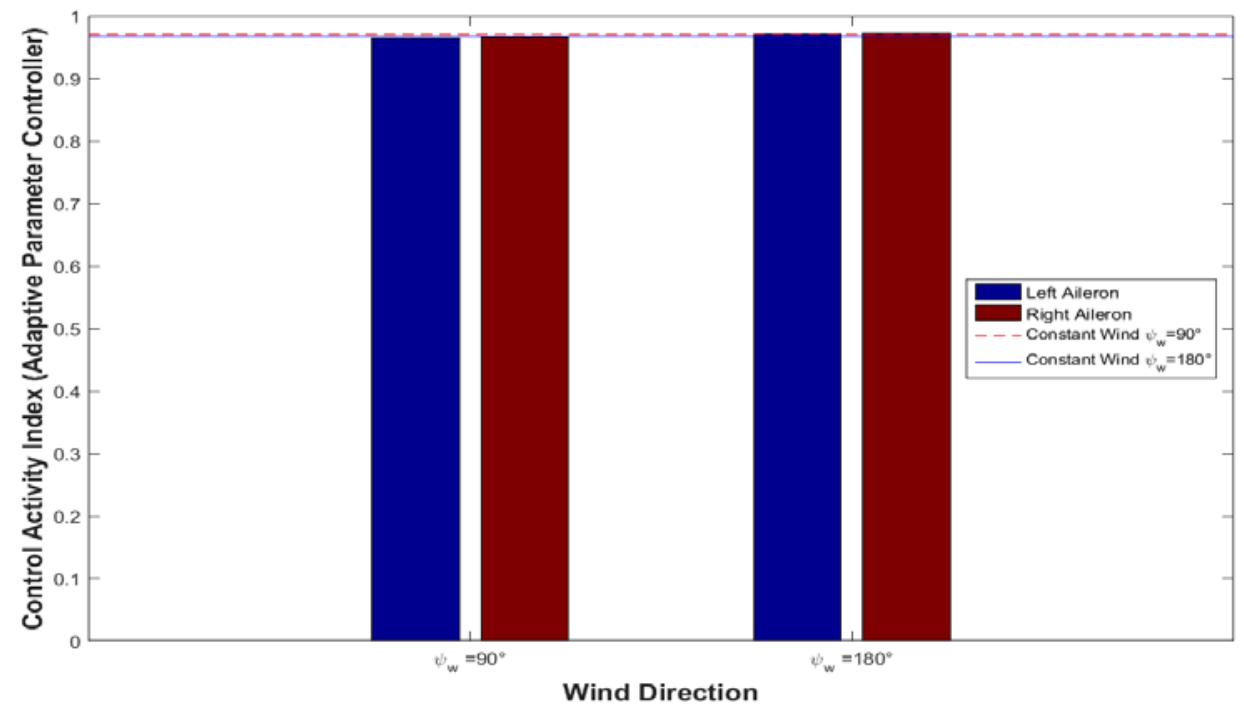

Figure 71- Control Activity for Under Constant Wind and Aileron Failure (Adaptive PPID).

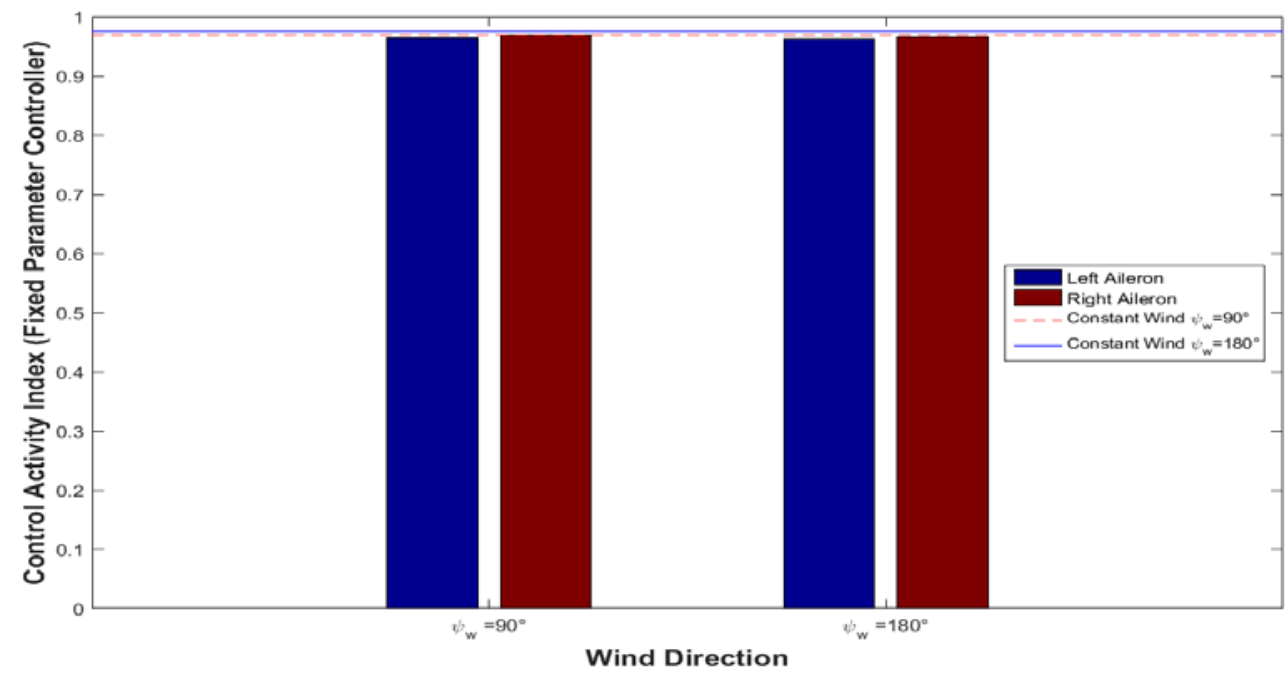

Figure 72- Control Activity for Under Constant Wind and Aileron Failure (Fixed PPID).

The trajectory tracking performance for wind gust combined with stabilator failure, presented a higher index for adaptive control laws than for fixed parameter control laws, as expected. However, the degradation for both controllers was expressive in comparison with performance indexes obtained under wind gust without any failure. Figure 73 illustrates this concept. 


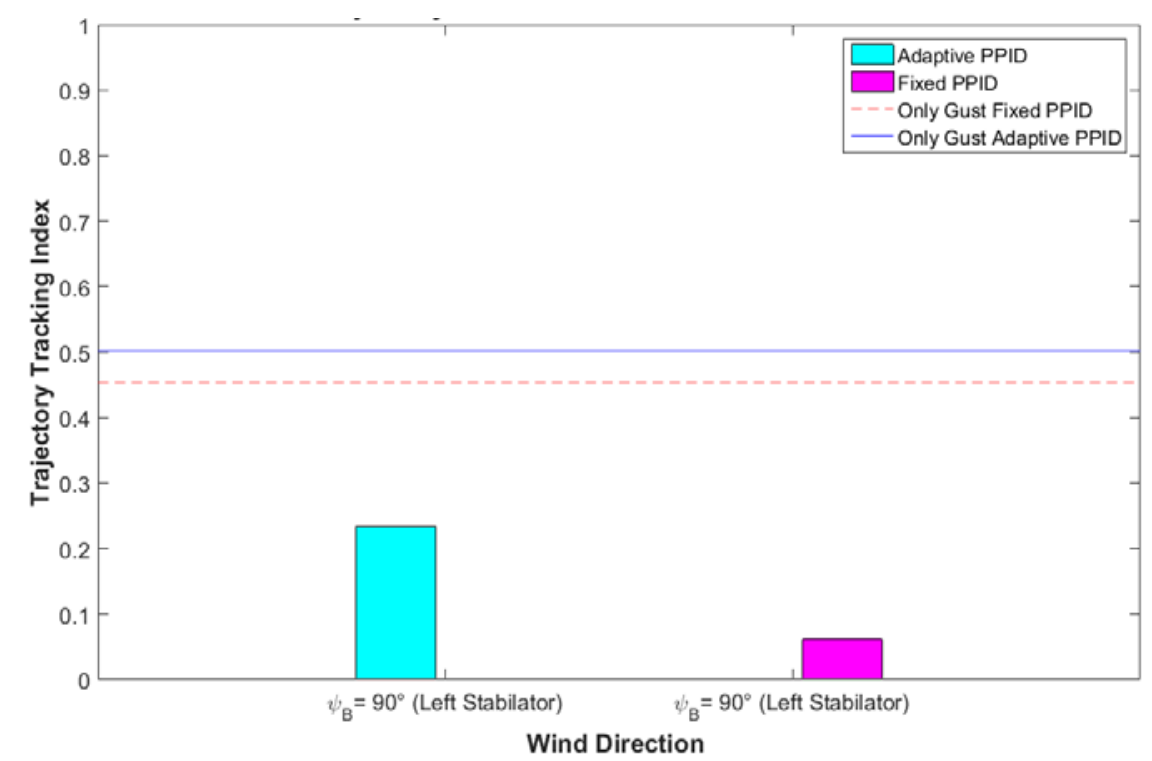

Figure 73- Tracking Index for Oval Trajectory Under Wind Gust and Stabilator Failure.

Although there was notable difference for trajectory tacking performance between adaptive and fixed parameter control laws, no relevant difference was found for control activity. This can be observed in Figure 74 .

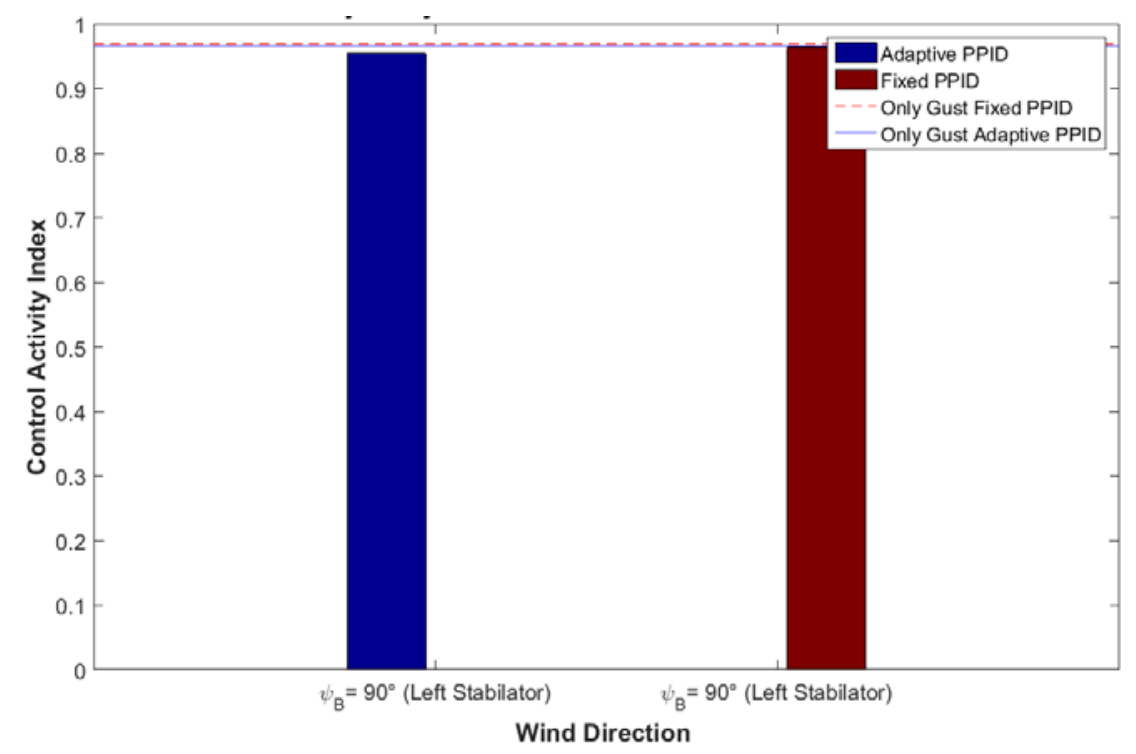

Figure 74- Control Activity for Oval Trajectory Under Wind Gust and Stabilator Failure.

Constant wind in combination with stabilator failure followed previous trends encountered for wind gust. The adaptive controller presented a higher trajectory tracking performance index than the fixed parameter controller for wind initially blowing at $\Psi_{w}=90^{\circ}$. Moreover, no relevant difference between the controllers was found for wind initially blowing at 
$\Psi_{\mathrm{w}}=180^{\circ}$. However, the adaptive and fixed parameter controllers presented significant degradation on trajectory tracking index in comparison with constant wind without any failure. This trend can be observed in Figure 75.

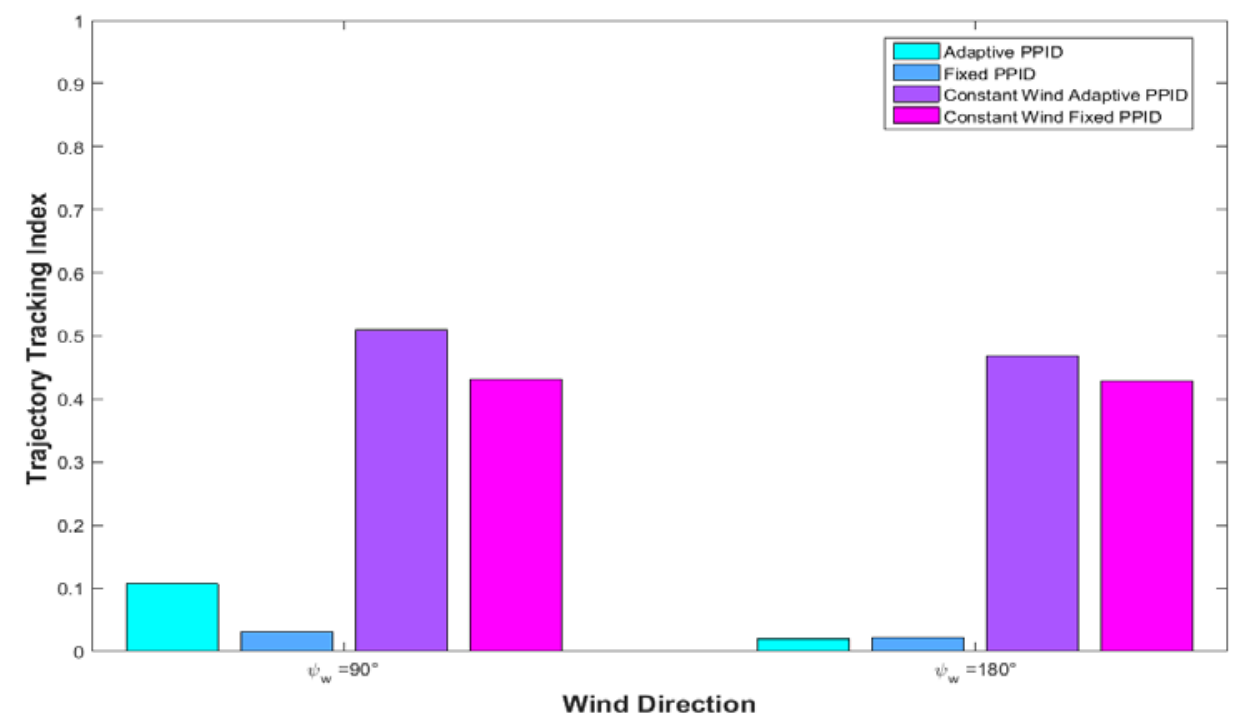

Figure 75- Tracking Index for Oval Trajectory Under Constant Wind and Stabilator Failure.

The adaptive parameter controller presents the lowest performance index for control activity, as expected. Although, the difference is very small if compared to the fixed parameter controller. Figure 76 demonstrates this.

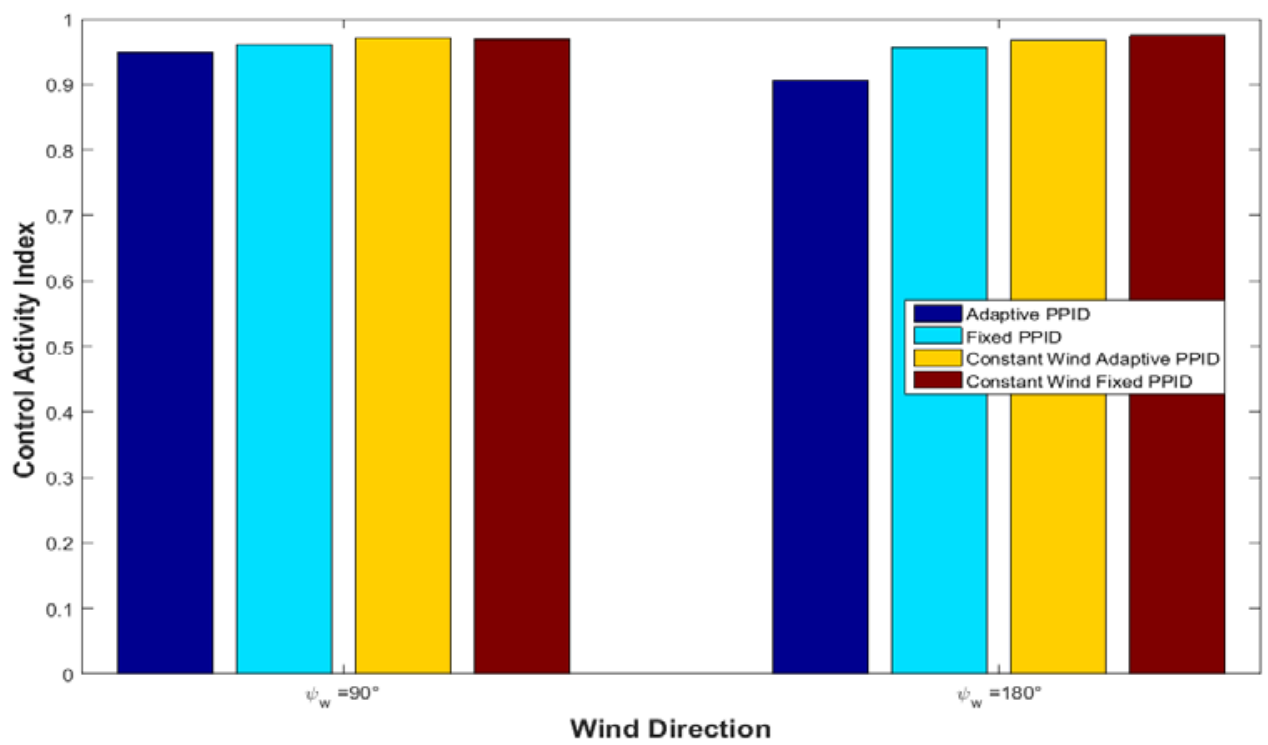

Figure 76- Tracking Index for Oval Trajectory Under Constant Wind and Stabilator Failure. 


\section{CONCLUSIONS AND FUTURE WORK}

This research effort had the objective of creating a wind model to better understand the effects of atmospheric phenomena on different UAVs trajectory tracking controllers. This goal was met with the development and implementation of a wind model for constant wind, wind gusts and wind shear into the WVU UAV simulation environment. Several analyses were performed using two different controllers of different complexity and different commanded trajectories. The effects of constant wind combined with different levels of turbulence, wind gusts and wind shear were investigated using extended simulation grid. Moreover, aileron and stabilator failures were introduced in addition to constant wind and wind gust for a wider range of case scenarios.

Notable degradation of trajectory tracking performance was recorded for all sets of control laws under wind phenomena, while control activity exhibited less sensitivity. In general, adaptive control laws performed better than fixed parameter ones, as expected. However, the relative degradation with respect to nominal conditions of the adaptive control laws performance was greater than for the fixed parameter control laws. These results lead to the observation that certain types of adaptive control laws may not necessarily exhibit the same level of effectiveness under wind as in the case of nominal conditions or other types of abnormal conditions.

Different trajectories had an effect on the performance index values evaluated for both types of controllers. The effects are correlated to the changes in the relative direction of the wind with respect to the aircraft imposed by the commanded trajectory. For example, the 3D S turns trajectory presents the most leveled performance indices for all wind phenomena in comparison with other trajectories.

A downward wind component typically degrades the trajectory tracking performance, while an upward component has the opposite effect. If the magnitude of the downward wind is increased, the performance index for trajectory tracking may degrade up to the point of loss of control. This trend was observed for the majority of the trajectories, which could be due to the aerodynamic effect on control effectiveness due to decreased aircraft angle of attack, in the downward wind case, as compared to increased angle of attack, in the upward wind scenario. However, further analysis must be performed in order to confirm the root cause of such trends and their generality. Under high speed constant wind, as the turbulence severity was increased, the performance indexes got worse for almost all cases, for both types of controllers. 
Analysis done for wind gust influence showed that a strong downward gust will always result in poor performance and often in loss of control. If the gust occurs during straight flight, performance is typically better than for the case when the gust occurs during turn. Regarding the duration of the gust, there is small difference between short and long duration gust for the fixed parameter controller. However, for the adaptive controller, long duration gust resulted in lower performance indices.

For wind shear, the vector component along the $Z$ axis, presents the lowest performance indices. Wind shear effect for vector component along the $Y$ axis results in small variation of performance indices, with the smallest index being in the gradient direction of the $Y$ axis. Moreover, analysis of the wind shear effect over vector component along the $\mathrm{X}$ axis, showed that the lowest performance was obtained for gradient in the $X$ axis direction. When the magnitude of the wind was increased, zero index value (and possible loss of control of the aircraft) was obtained. The gradient with respect to $Z$ for all components should not vary with exception of the 3D turns, because it is the only trajectory that has significant altitude variation. With respect to location of the wind shear (turn or straight flight), straight flight presented slightly higher performance indices.

Aircraft failures in the left or right aileron of limited severity, in addition to low speed wind gust, did not affect the tracking performance. Under constant wind in addition to aileron failures, the performance indices obtained for the adaptive parameter controller were lower than performance index values obtained for constant wind only. On the other hand, for the fixed parameter controller, the performance indices were oddly higher than values obtained with no failure. This could be associated with the flight path, where a deflection of the aileron would facilitate for the aircraft to turn, although, a more thorough analysis should be done to conclude the root cause. The performance index values for left aileron failure are higher than for right aileron failures. This could be due to the trajectory where the aircraft only turns right. For control activity there was no relevant difference between failures for both controllers, this holds for wind gust or constant wind. For failures in the left stabilator combined with constant wind or wind gust, the adaptive controller presented higher trajectory tracking performance indexes than the fixed parameter controller. Regarding control activity, the difference between the two control laws was small; however, the adaptive control laws presented slightly lower performance indices.

With the conclusion of this research effort, analysis of atmospheric phenomena on trajectory tracking control laws was conducted. Currently, the trajectory tracking control laws investigated, while performing well under several cases of abnormal flight conditions, did not 
perform as effectively under wind conditions. Thus, UAV flight under wind phenomena poses a challenge regarding the design of trajectory tracking control laws.

Wind phenomena are a danger for aviation and it is essential that autonomous aircraft are able to fly safely under such influence. Thus, for future work, this research could be used as basis to develop trajectory tracking controllers specifically aimed at handling wind phenomena. Future research should investigate more thoroughly the combined effects of wind phenomena and failures of aircraft subsystem, such as actuators, engine, and sensors, including GPS. Moreover, the wind phenomena GUIs should be implemented in the main GUI of the WVU UAV simulation environment to allow for faster simulation inputs.

The simulations cases presented were performed one single time each. In a real environment, several tests should be performed under the same conditions for statistical analysis purposes. However, since the simulations were performed in a computational environment, no variations between similar tests occurred. Only the turbulence model has a random component. However, the statistical properties of the simulated turbulence as a random process are consistently the same.

An analysis of variance (ANOVA) could be performed in future work to more clearly identify and confirm the most important factors and to better quantify their effects. 


\section{REFERENCES}

[1] Federal Aviation Administration (FAA), "Unmanned Aircraft Systems", retrieved from: www.faa.gov/uas/ in Aug 29, 2016.

[2] The UAV, "UAVs", retrieved from: www.theuav.com/ in Aug, 2017.

[3] Wen-Hua, L. C. C., "Disturbance Rejection Flight Control for Small Fixed-Wing Unmanned Aerial Vehicles", vol. 39, pp. 2804-2813, 2016.

[4] Wilburn, B. K., "Fault-Tolerant Trajectory Tracking of Unmanned Aerial Vehicles Using Immunity-Based Model Reference Adaptive Control", MS Thesis, West Virginia University, Dec 5, 2014.

[5] Al-Sinbol, G., "Analysis of GPS Abnormal Conditions Within Fault Tolerant Control Laws", MS Thesis, West Virginia University, Jan 1, 2013.

[6] Karas, O., "UAV Simulation Environment for Autonomous Flight Control Algorithms", MS Thesis, West Virginia University, Jan 1, 2012.

[7] Perhinschi, M. G., Wilburn, B., Wilburn, J., Moncayo, H., Karas, O., "Simulation Environment for UAV Fault Tolerant Autonomous Control Laws Development", Journal of Modeling, Simulation, Identification, and Control, vol. 1, pp. 164-195, 2013.

[8] CENIPA, "Panorama Estatistico Da Aviacao Brasileira", CENIPA, pp. 37-45, 2016.

[9] CENIPA, "Relatorios Finais", CENIPA, 2017.

[10] ISO 2533, "International Standard Atmosphere", 1962.

[11] Frost, D., Camp, D., "Wind Shear Modeling for Aircraft Hazard Definition", 1977.

[12] National weather service, "Origin of Wind", retrieved from: www.weather.gov/in Oct, 2016.

[13] Committee, "Low-Altitude Wind Shear and Its Hazard to Aviation", National Academies Press, 1983.

[14] National Transportation Safety Board Bureau of Accident investigation, "Aircraft Accident Report Pan American World Airways, Clipper 759", 1983.

[15] Smith, M., "Defeating The Downburst: 20 Years Since Last U.S. Commercial Jet Accident From Wind Shear", Jul 2, 2014.

[16] U.S. Department of Transportation, "Eastern Airlines Flight 66, B727 ", Jun 24, 1975. 
[17] Moser, W., "Ted Fujita, Chicago's Mr. Tornado, and The Super Outbreak of 1974", Apr 28, 2011.

[18] Office of System Safety, "Review of Aviation Accidents Involving Weather Turbulence In The United States 1992-2001", NASA/Langley Research Center, 2004.

[19] BBC News, "Queens Crash Blamed on Co-pilot", Oct 26, 2014.

[20] Kozo Mizoguchi -The Associated Press, "Jet Turbulence Leaves 1 Dead, Scores Injured", Greensboro News Record, Dec 29, 1997.

[21] Belcastro, C., Foster, J., "Aircraft Loss-of-Control Accident Analysis", Jan 1, 2010.

[22] National Transportation Safety Board, "Loss of pitch control on takeoff Emery Worldwide Airlines, Flight 17 McDonnell Douglas DC-8-71F, N8079U Rancho Cordova, California, February 16, 2000", 2003.

[23] Comision Investigadora de Acidentes de Aviacion, "Informe Final De Accidente De Aviacion McDonnel Douglas DC - 9-32 Austral, Lineas Aereas Cielos Del Sur S.A.", 2017.

[24] National Earth Science Teachers Association (NESTA), "Earth's Atmosphere", 2012.

[25] Pena, R. F. A., "Tipos De Vento", retrieved from: alunosonline.uol.com.br in Sep, 2016.

[26] The Editors of Encyclopædia Britannica, "Gustave-Gaspard Coriolis", Jul 30, 2008.

[27] Silvia, M., "Natural Disasters: Key Concepts", Mar, 2010.

[28] Pidwirny, M., "Fundamentals of Physical Geography", 2006.

[29] Glenn Research Center, "Relative Velocities", retrieved from: www.grc.nasa.gov, Sep, 2016.

[30] Soares, P. M., "Ventos", CMTE blog, Jul 07, 2008.

[31] The editors of Encyclopaedia Britannica, "Updrafts and Downdrafts", Oct, 2001.

[32] Charlton, C., Duell, M., "It Must Be Windy If The World's Biggest Airliner Is Blown off Course", retrieved from: www.Dailymail.Co.Uk, Jan, 2015.

[33] Weather Guys Editor, "What Causes Wind Gusts?", Jan, 2012.

[34] Weather Underground, "Wind Gusts vs. Gust Speed", Oct, 2006.

[35] National Weather Service, "Thunderstorm Hazards - Damaging Wind", retrieved from: www.srh.noaa.gov in Nov, 2016. 
[36] Léonard, F., Martini, A., Abba, G., "Robust Nonlinear Controls of Model-Scale Helicopters Under Lateral and Vertical Wind Gusts", Transactions on Control Systems Technology, vol. 20, pp. 154-163, Jan, 2012.

[37] Richardson, J., Atkins, E., Kabamba, P., Girard, A., "Envelopes for Flight Through Stochastic Gusts", Proc. of AIAA Atmospheric Flight Mechanics Conference, Aug, 2011.

[38] Raza, S. A., "Autonomous UAV Control for Low-Altitude Flight in an Urban Gust Environment", Doctoral Dissertation, Carleton University, Aug, 2015.

[39] Gaonkar, G. H., "Gust Response of Rotor and Propeller Systems," Journal of Aircraft, vol. 18, pp. 389-396, May, 1981.

[40] FAA Safety Team, "Wind Shear", FAA (Federal Aviation Administration), 2008.

[41] Secretary General, "Manual on Low-level Wind Shear", ICAO, 2005.

[42] Beaudette, D. C., "Pilot Wind Shear Guide", Federal Aviation Administration (FAA), pp. 64, 1988.

[43] Rogers, A., Manwell, J., Ellis, A., "Wind Shear Over Forested Areas", American Institute of Aeronautics and Astronautics.

[44] Bochis, V., "Dynamics of An Aircraft In Wind Shear of Arbitrary Direction", Journal of Guidance, Control, and Dynamics, vol. 7, pp. 615-619, Sep. 1984.

[45] Frost, W., Bowles, R.L., "Wind Shear Terms In The Equations of Aircraft Motion", Journal of Aircraft, vol. 21, pp. 866-872, Nov, 1984.

[46] Etkin, B., "Comment on Wind Shear Terms In The Equations of Aircraft Motion", Science, vol. 24, pp. 477-479.

[47] Mulgund, S. S., Stengel, R. F., "Optimal Nonlinear Estimation for Aircraft Flight Control In Wind Shear", Automatica, vol. 32, pp. 3-13, Jan, 1996.

[48] Grillo, C., Montano, F., Patti, M., "Wind Shear on-Line Identification for Unmanned Aerial Systems", The Journal of Aerospace Science, Technology and Systems, vol. 93, Dec, 2014.

[49] Anderson, J. D., "Fundamentals of Aerodynamics", New York, NY: McGraw-Hill, 2017, pp. 1051-1078.

[50] Richard, D., Hallion, P., "Flight Environment Operations Flight Testing and Research", Library of Congress Cataloging-in-Publication Data, 2010. 
[51] G. Kulesa, G., "Weather and Aviation: How Does Weather Affect the Safety and Operations of Airports and Aviation, and How Does FAA Work to Manage Weather-related Effects? ", Federal Aviation Administration (FAA).

[52] NASA Dryden Fact Sheet, "Airborne Coherent LIDAR for Advanced In-Flight Measurement", 2005.

[53] Beal, T. R., "Digital Simulation of Atmospheric Turbulence for Dryden and von Karman Models", Journal of Guidance, Control and Dynamics, vol. 16, Jan, 1993.

[54] Watkins, S., Milbank, J., Loxton, B. J., Melbourne, W.H., "Atmospheric Winds and Their Implications for Microair Vehicles", AIAA Journal, vol. 44, pp. 2591-2600, Nov, 2006.

[55] Staveren, V., Jan Joseph, W. H., "Analyses of Aircraft Responses to Atmospheric Turbulence", Dec, 2003.

[56] Perhinschi, M. G., Napolitano M. R., Tamayo S., "Integrated Simulation Environment For Unmanned Autonomous Systems - Towards A Conceptual Framework", Modelling and Simulation in Engineering, Volume 2010 (2010), Article ID 736201, 12 pages, doi:10.1155/2010/736201, 2010 .

[57] Perhinschi, M. G., Beamer F., "Flight Simulation Environment for Undergraduate Education in Aircraft Health Management", Computers in Education Journal, Vol. 22, No. 3, pp. 50-62,JulySep, 2012.

[58] Etkin, B., “Dynamics of flight", New York: Wiley, 1982.

[59] Kane, T.R., Levinson, D.A. “Dynamics: Theory and Application”, McGraw-Hill, 1985.

[60] Etkin, B., "Turbulent Wind and Its Effect on Flight", J. Aircraft, vol. 18, pp. 327-345, May, 1981.

[61] Wilburn, J. N., "Development of an Integrated Intelligent Multi -Objective Framework for UAV Trajectory Generation", Doctoral Dissertation, West Virginia University, Aug, 2013.

[62] Word Press, "Introduction - FlightGear Flight Simulator", retrieved from: www.flightgear.org in Jan, 2017.

[63] Al-Sinbol, G., Perhinschi, M. G., Wilburn, B. K., "Simplified GPS Model for UAV Fault Tolerant control laws design", International Journal of Intelligent Unmanned Systems, vol. 03, pp. 39-60, 2015. 
[64] Napolitano, M. R. "Development of Formation Flight Control Algorithms Using 3 YF-22 Flying Models", DTIC Document, 2005.

[65] Perhinschi, M. G., Napolitano, M. R., Campa, G., Seanor, B., Burken, J., Larson, R., "An Adaptive Threshold Approach for The Design of an Actuator Failure Detection and Identification Scheme", Transactions on Control Systems Technology, vol. 14, May, 2006.

[66] Perhinschi, M.G., Moncayo, H., Al Azzawi, D., "Integrated Immunity-Based Framework for Aircraft Abnormal Conditions Management", Journal of Aircraft, vol. 51, pp. 1726-1739, Nov, 2014.

[67] Krishnamoorthy, K., "Fault Tolerance Analysis of L1 Adaptive Control System for Unmanned Aerial Vehicles", MS Thesis, West Virginia University, Jan 1, 2015.

[68] The Weather Channel, "US Winds and Gusts", retrieved from: www.weather.com in Jan, 2017. 
APPENDIX A - SIMULATION PLOTS 


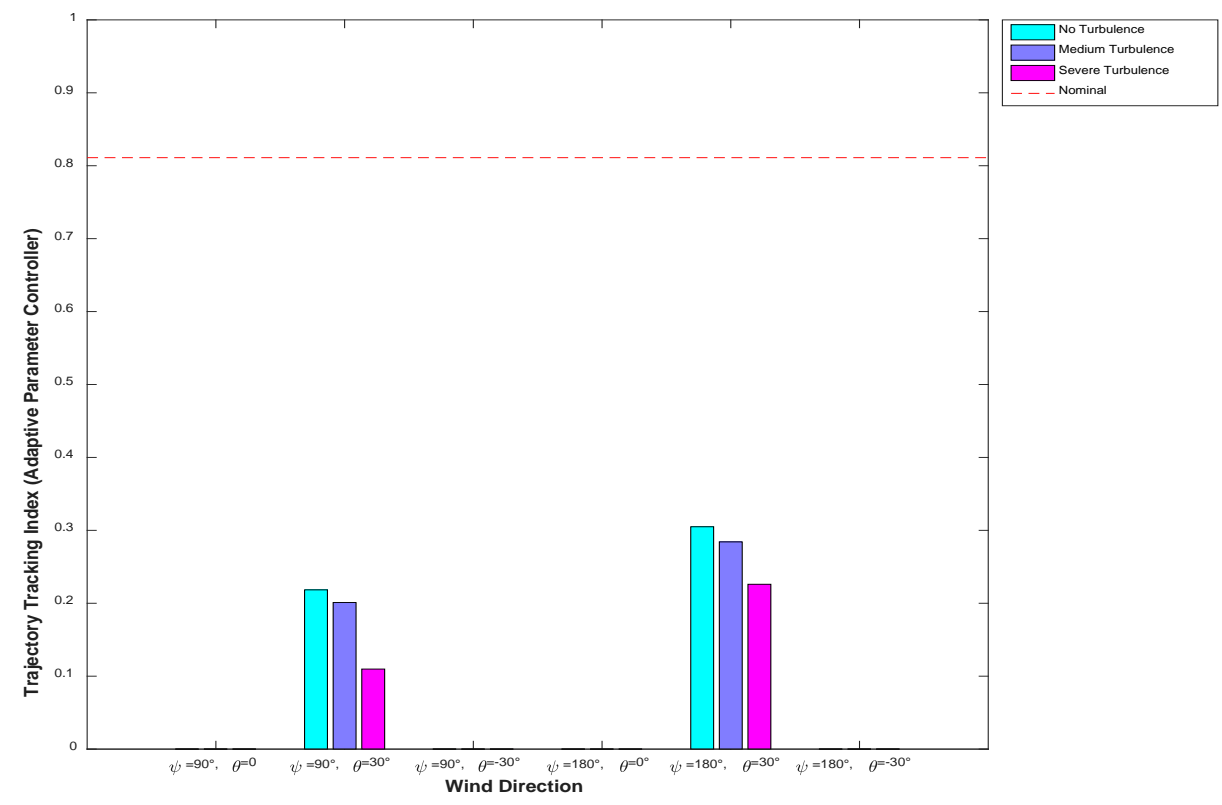

Figure A- 1- Clothoid Trajectory Index Under Strong Constant Wind (Adaptive PPID).

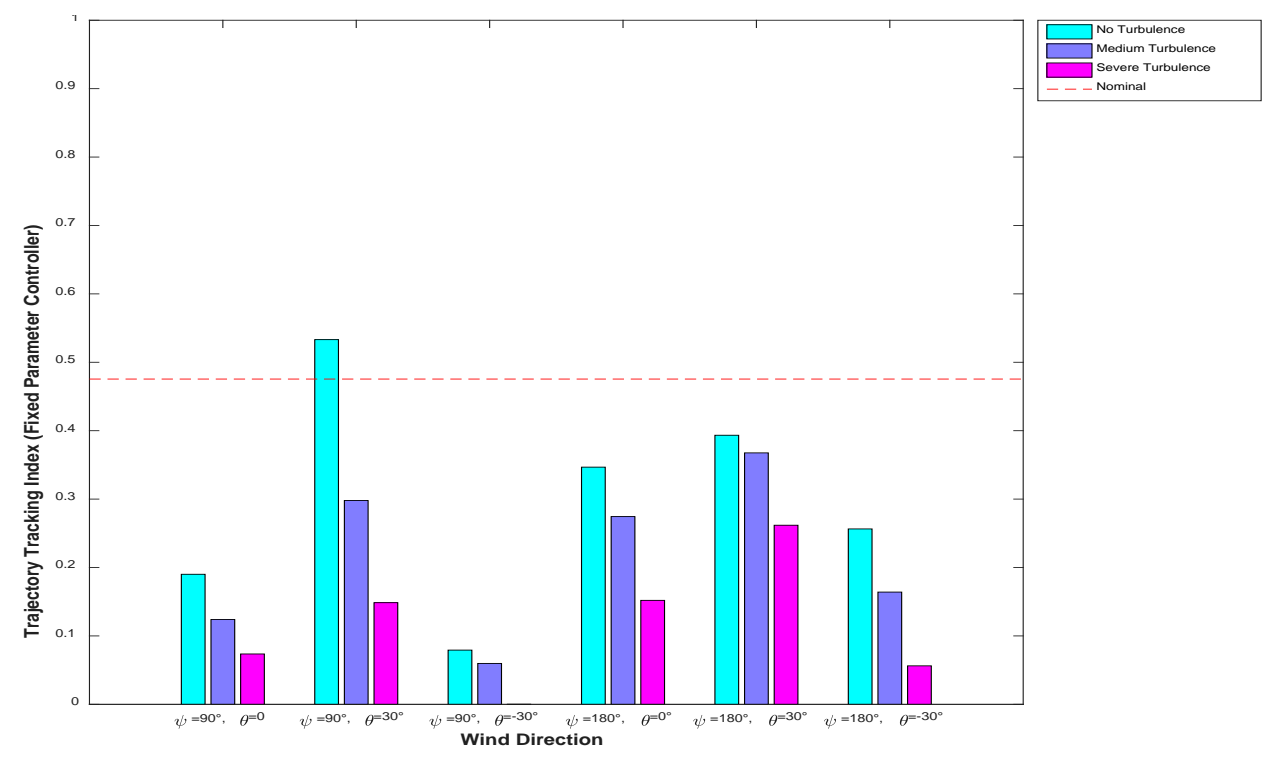

Figure A- 2 -Clothoid Trajectory Index Under Low Constant Wind (Fixed PPID). 


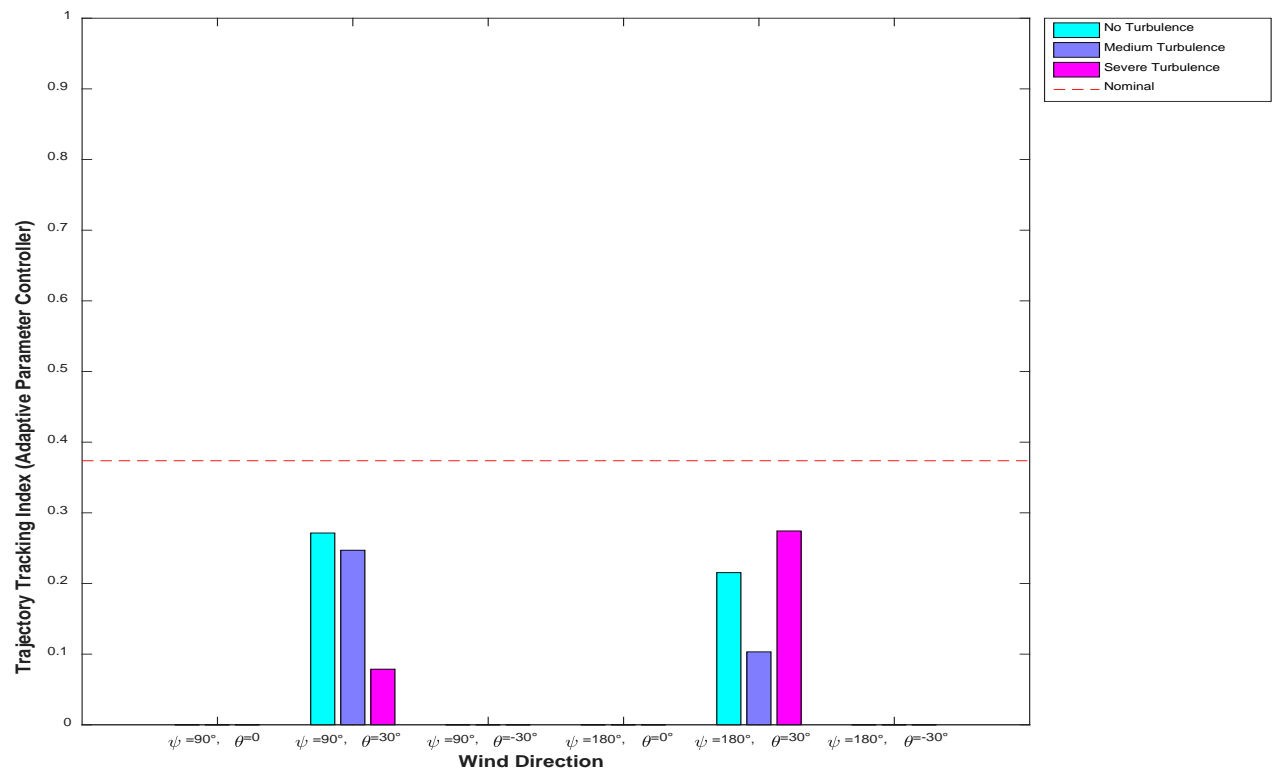

Figure A- 3 Dubins Trajectory Index Under Strong Constant Wind (Adaptive PPID).

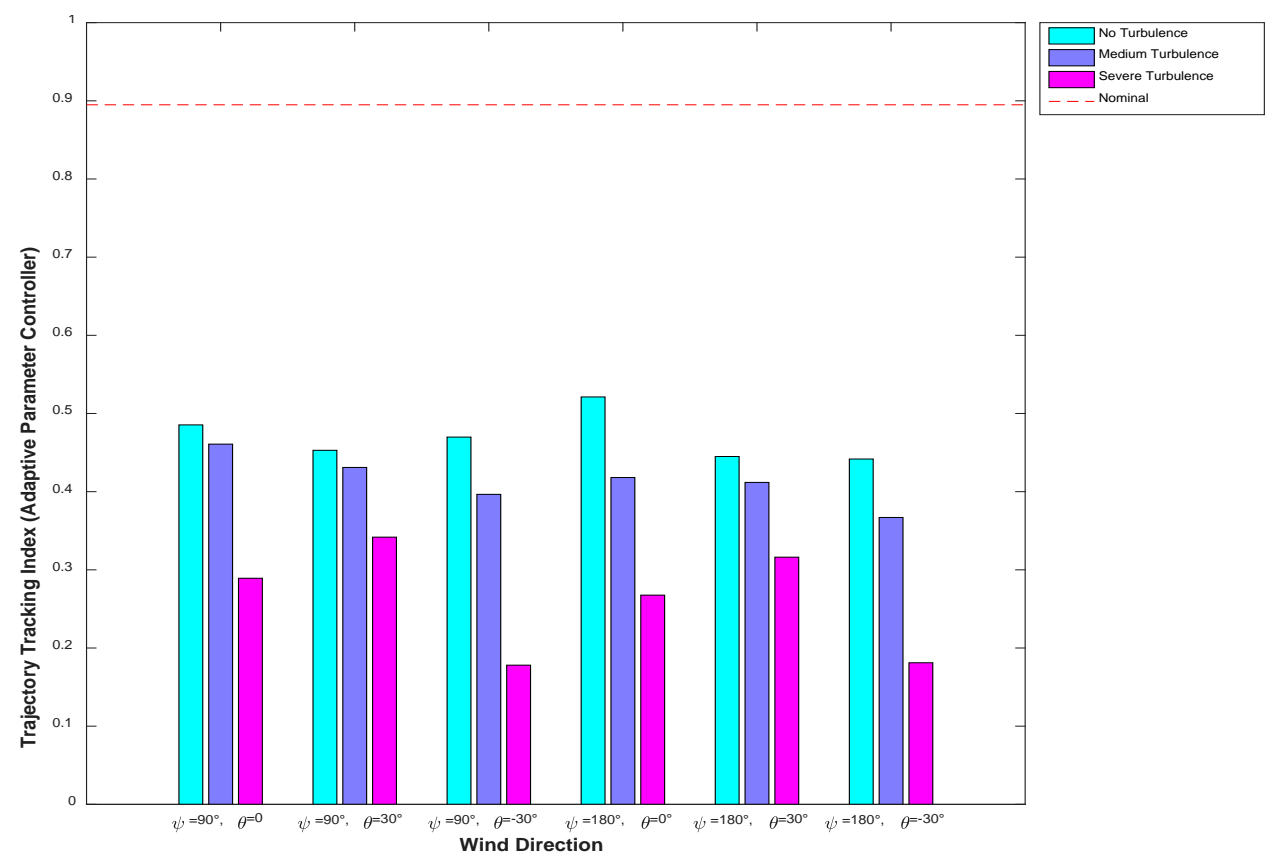

Figure A- 4- Figure 8 Trajectory Index Under Low Constant Wind (Adaptive PPID). 


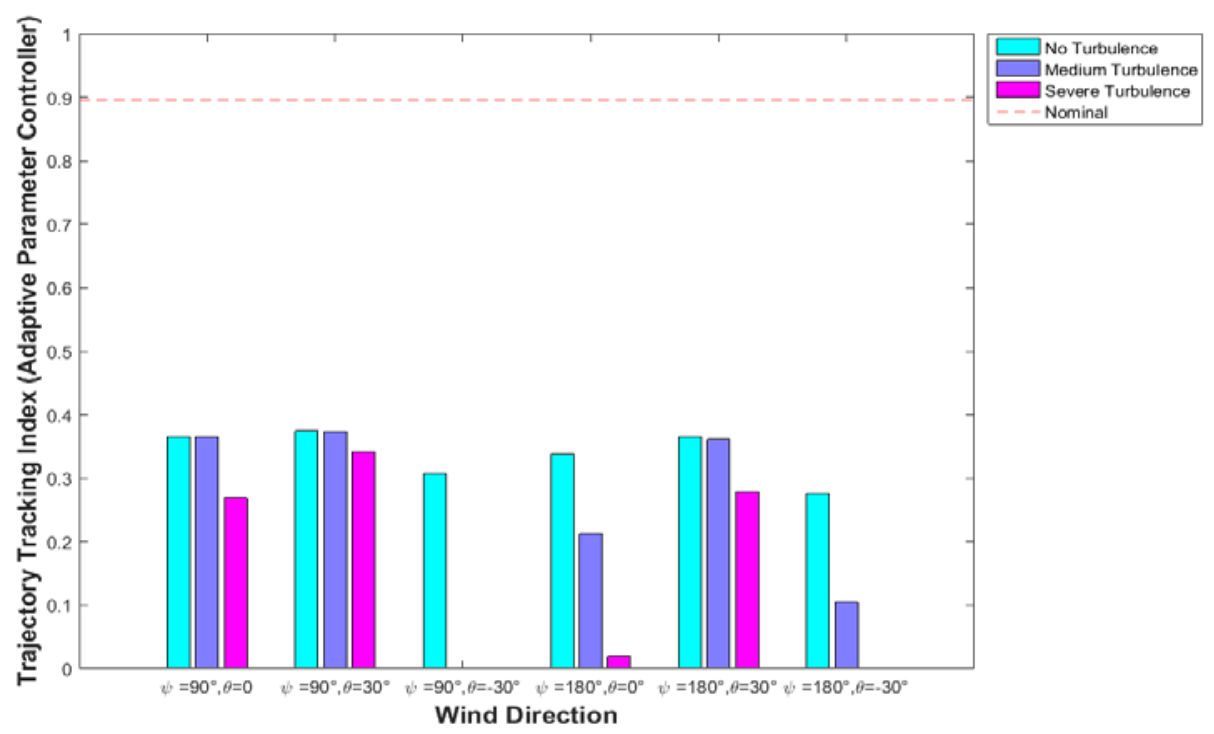

Figure A- 5- Figure 8 Trajectory Index Under Strong Constant Wind (Adaptive PPID).

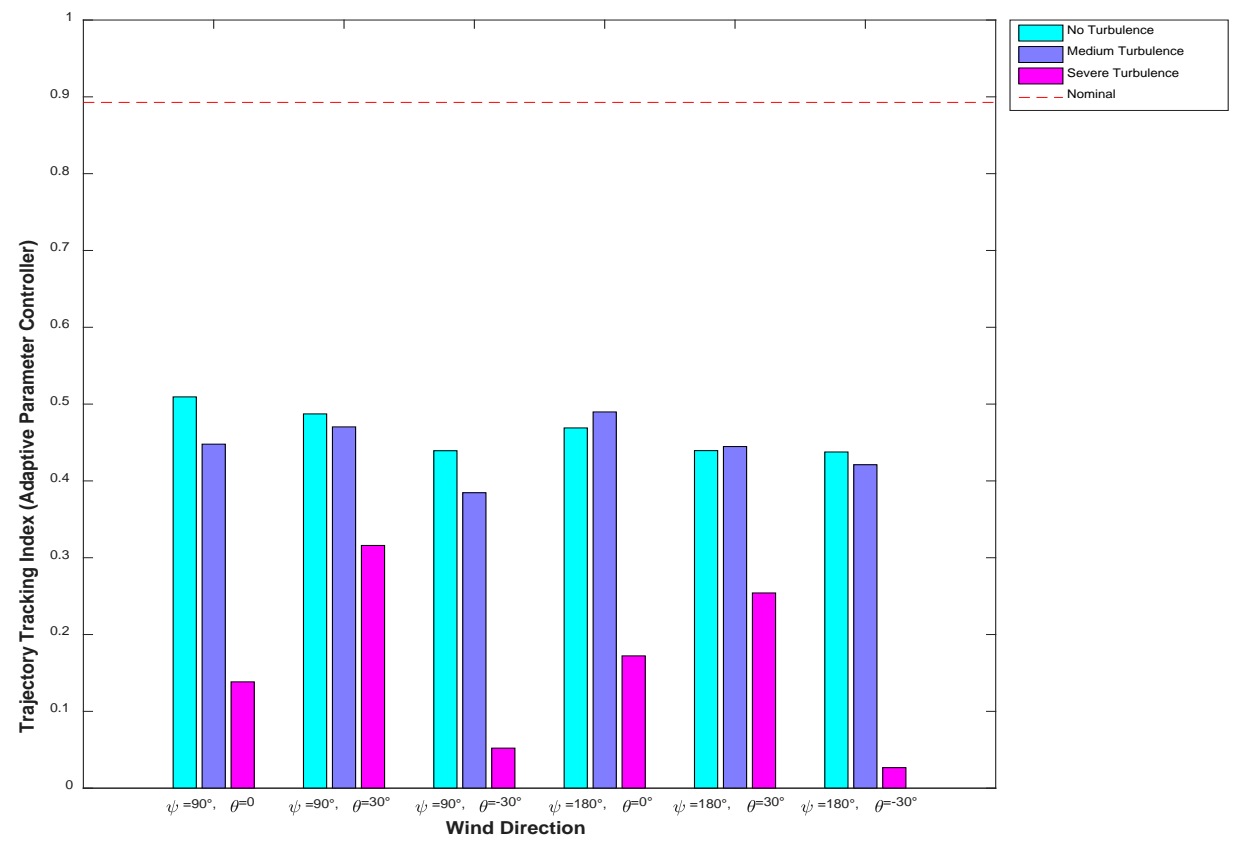

Figure A- 6- Oval Trajectory Index Under Low Constant Wind (Adaptive PPID). 


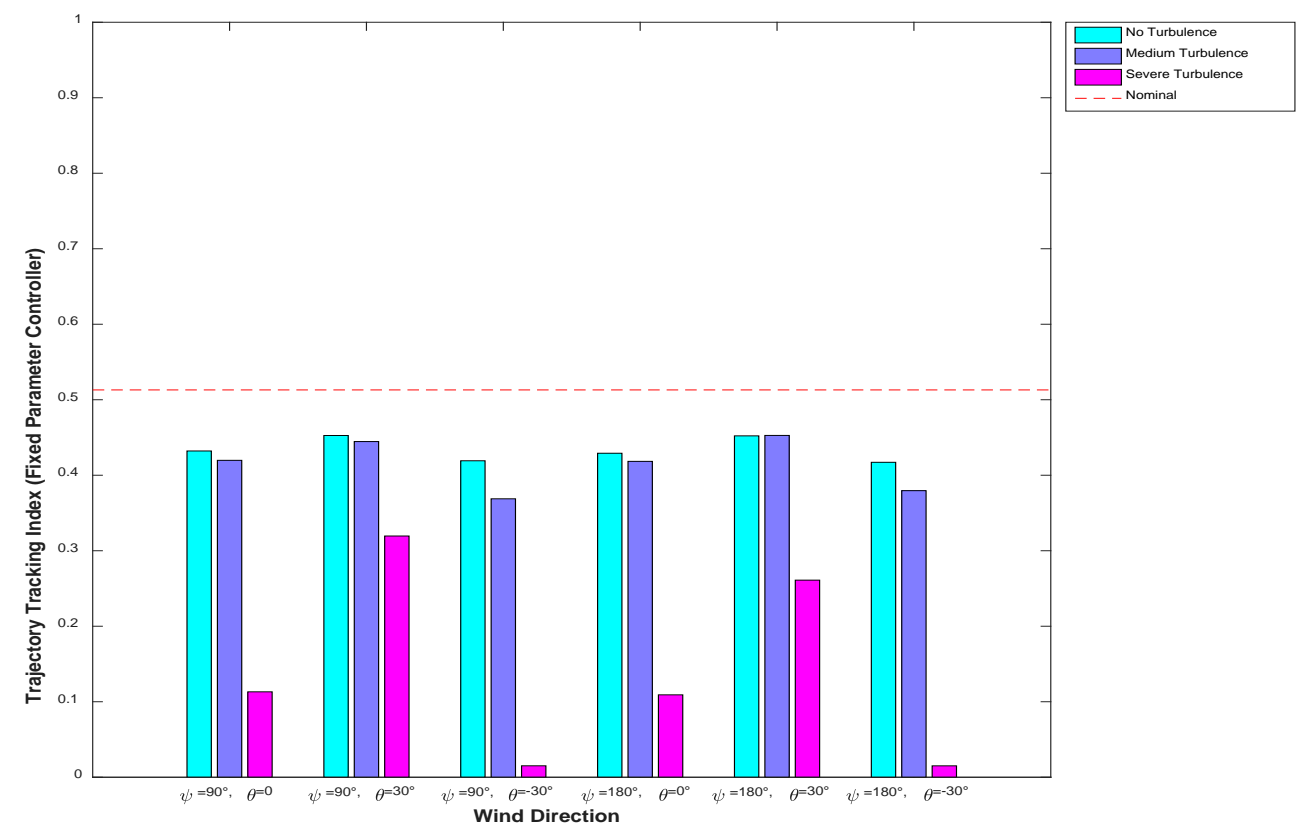

Figure A- 7- Oval Trajectory Index Under Low Constant Wind (Fixed PPID).

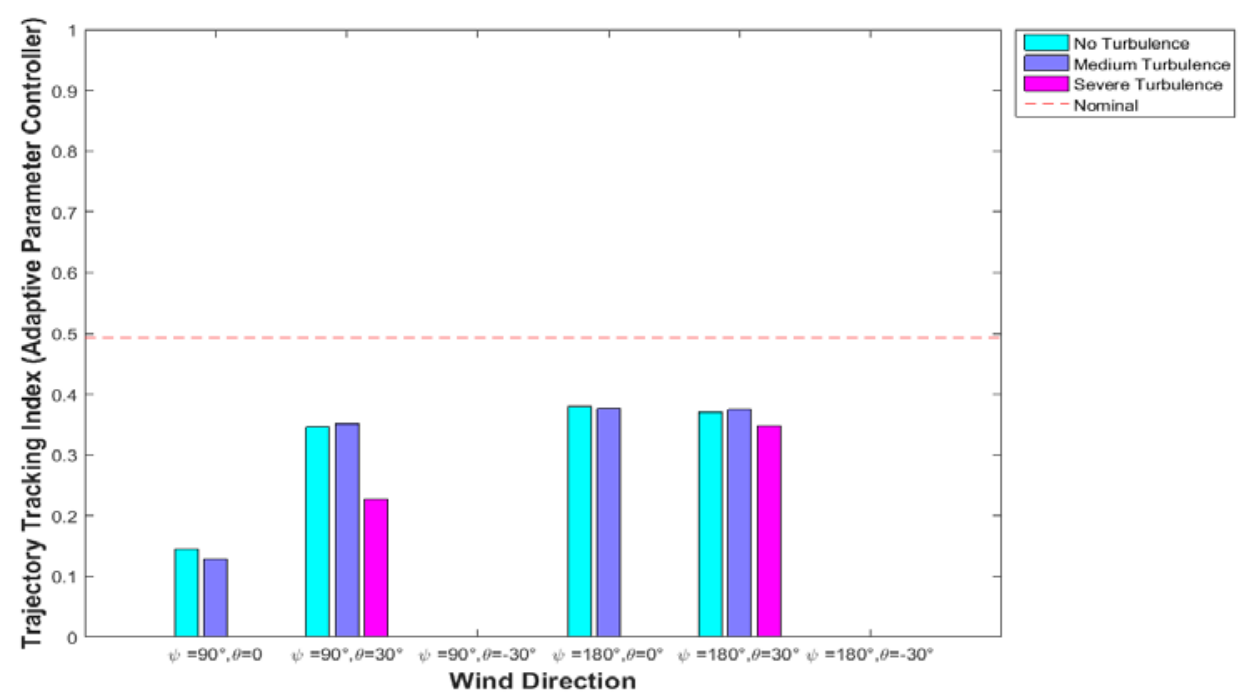

Figure A- 8- 3D S Turns Trajectory Index Under Strong Constant Wind (Adaptive PPID). 


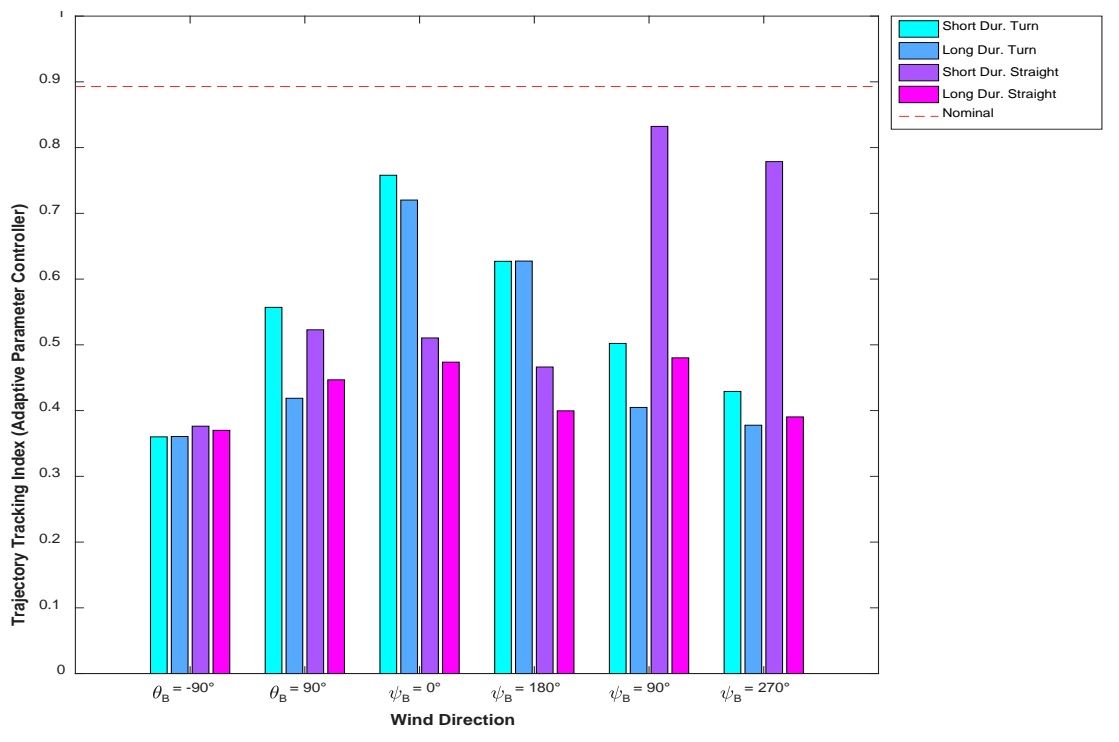

Figure A- 9- Oval Trajectory Index Under Low Wind Gust (Adaptive PPID).

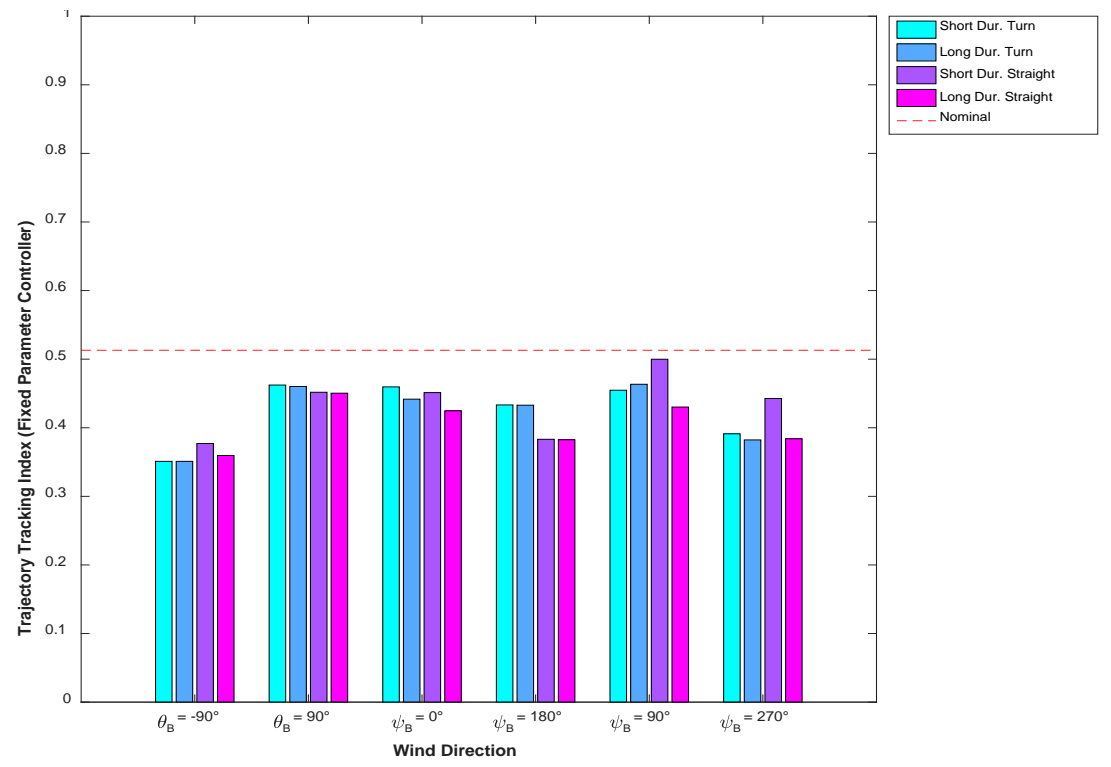

Figure A- 10 Oval Trajectory Index Under Low Wind Gust (Fixed PPID). 


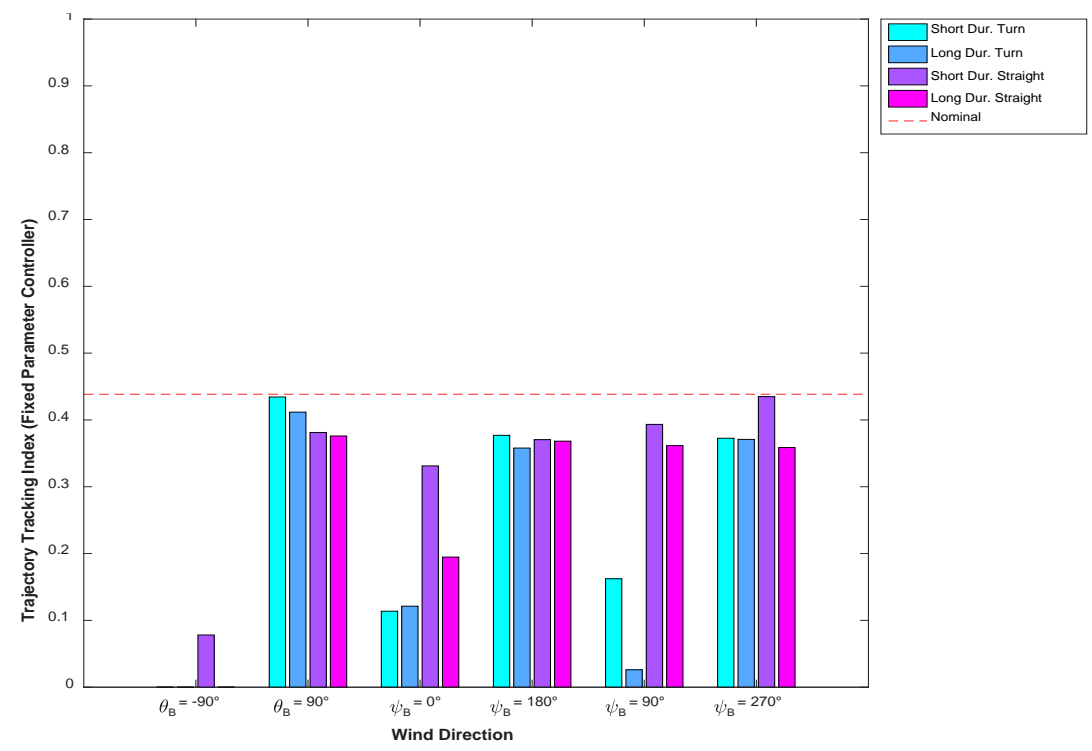

Figure A- 11- 3D S Turns Trajectory Index Under High Wind Gust (Fixed PPID).

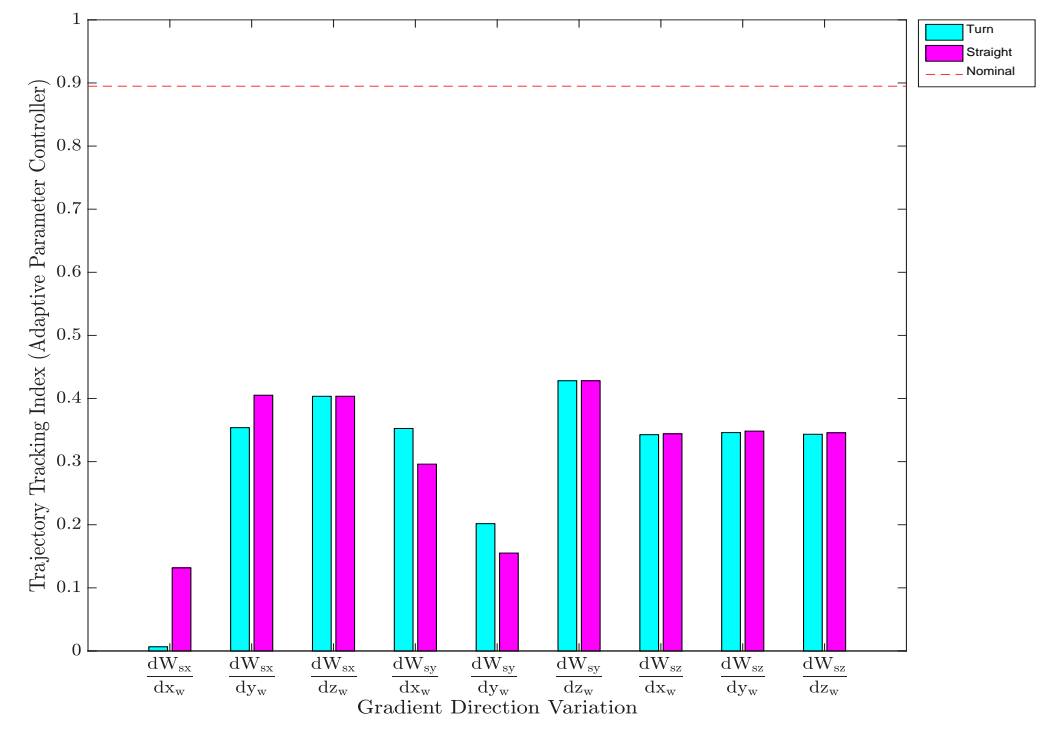

Figure A-12- Figure 8 Trajectory Index Under Strong Wind Shear (Adaptive PPID). 


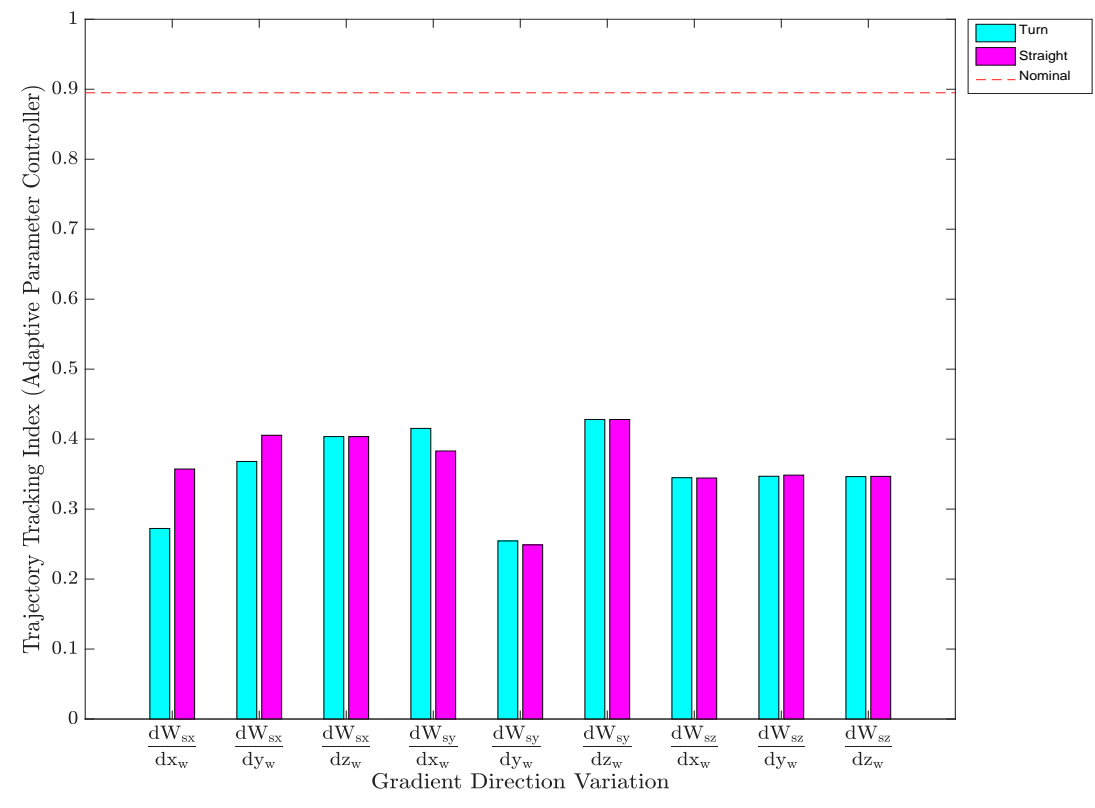

Figure A- 13- Figure 8 Trajectory Index Under Low Wind Shear (Adaptive PPID).

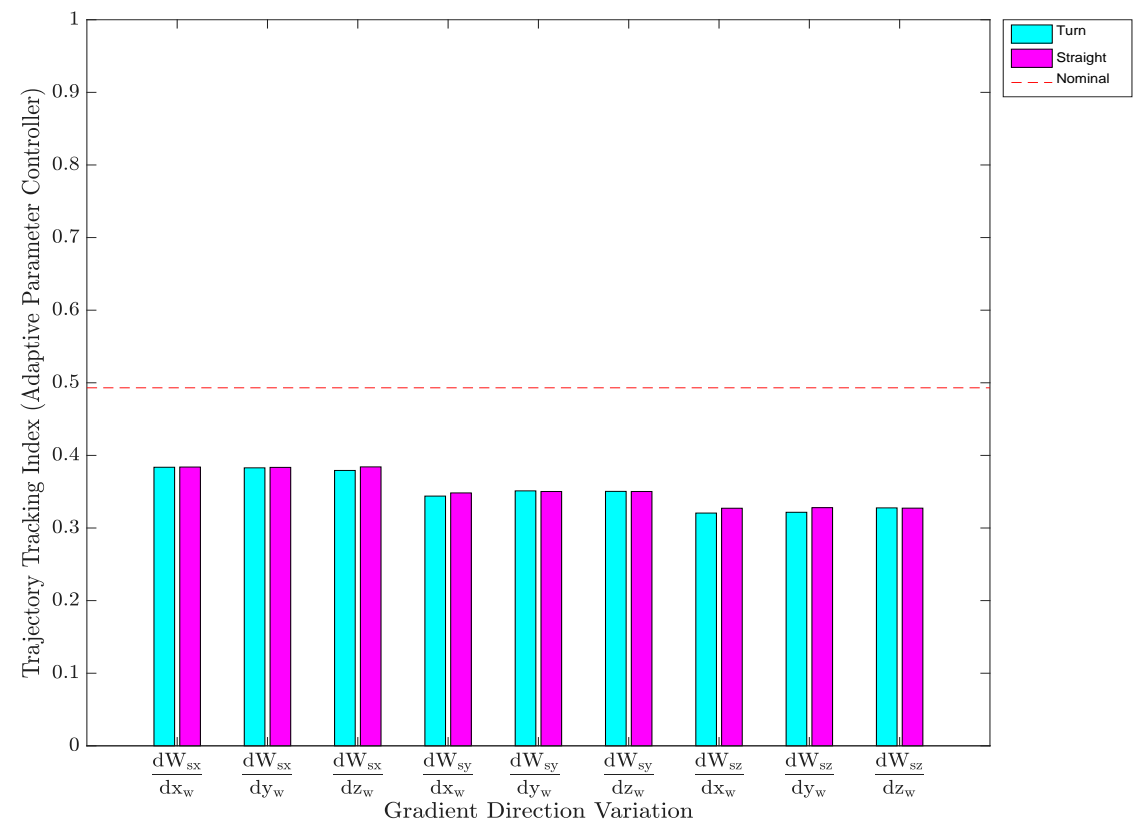

Figure A-14-3D S Turns Trajectory Index Under Low Wind Shear (Adaptive PPID). 